Supporting Information

for

\title{
Neutral dinuclear copper(I)-NHC complexes: synthesis and application in the hydrosilylation of ketones
}

Michael Trose, ${ }^{a}$ Faïma Lazreg, ${ }^{a}$ Tao Chang, ${ }^{a}$ Fady Nahra, ${ }^{b}$ David B. Cordes, ${ }^{a}$ Alexandra M. Z. Slawin $^{\mathrm{a}}$ and Catherine S. J. Cazin ${ }^{\mathrm{a}, \mathrm{b} *}$

a) EaStCHEM School of Chemistry, University of St Andrews, St Andrews, KY16 9ST (UK)

b) Ghent University, Department of Inorganic and Physical Chemistry, Krijgslaan 281, S-3, B-9000 Ghent, Belgium

E-mail: Catherine.Cazin@UGent.be 


\section{Table of Contents}

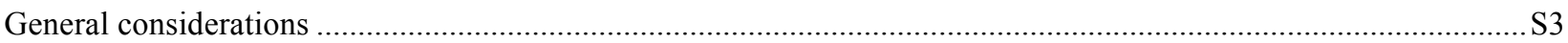

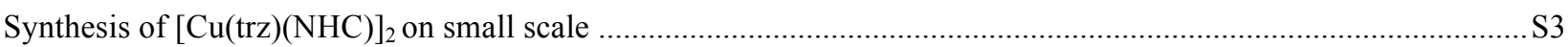

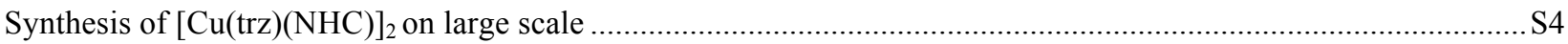

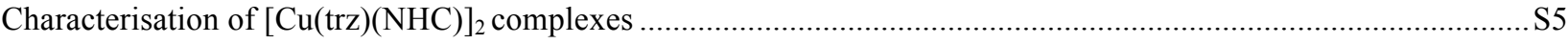

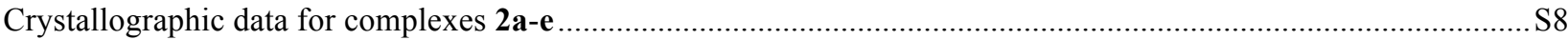

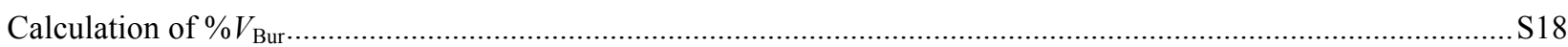

Hydrosilylation of ketones catalysed by dimeric copper complexes .................................................................... 26

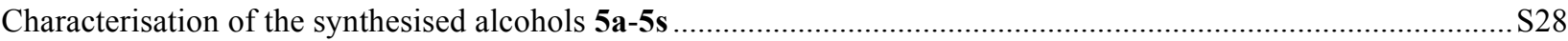

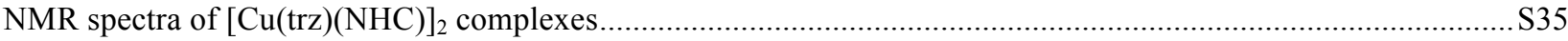

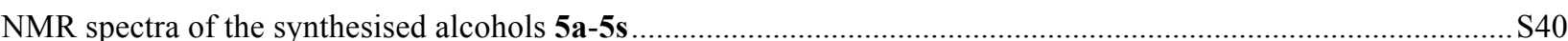




\section{General considerations}

All reactions were carried out under inert atmosphere of argon using a glovebox. All reagents were purchased and used as received. All solvents were dried before use. ${ }^{1} \mathrm{H}$ and ${ }^{13} \mathrm{C}-\left\{{ }^{1} \mathrm{H}\right\}$ Nuclear Magnetic Resonance (NMR) spectra were recorded at 298K on a Brucker Advance 400 Ultrashield or on a Brucker Advance 500 Ultrashield spectrometer using the residual solvent peak $\left(\mathrm{CDCl}_{3}: \delta_{\mathrm{H}}=7.26 \mathrm{ppm} ; \delta_{\mathrm{C}}=77.36 \mathrm{ppm} ; \mathrm{CD}_{2} \mathrm{Cl}_{2}: \delta_{\mathrm{H}}=5.32 \mathrm{ppm}, \delta_{\mathrm{C}}=54.3 \mathrm{ppm} ; \mathrm{C}_{6} \mathrm{D}_{6}: \delta_{\mathrm{H}}=\right.$ $\left.7.15 \mathrm{ppm}, \delta_{\mathrm{C}}=128.62 \mathrm{ppm}\right)$ or TMS as reference. Elemental analysis was performed by the London Metropolitan University. Gas chromatography (GC) analyses were performed on an Agilent 7890A apparatus equipped with a flame ionization detector and a (5\%-phenyl)-methylpolysiloxane column ( $30 \mathrm{~m}, 320 \mu \mathrm{m}$, film: $0.25 \mu \mathrm{m})$.

\section{Synthesis of $[\mathrm{Cu}(\mathrm{trz})(\mathrm{NHC})]_{2}$ on small scale}

\section{From $[\mathrm{Cu}(\mathrm{OH})(\mathrm{IPr})]$}

In a glovebox, a screw capped vial was charged with $[\mathrm{Cu}(\mathrm{OH})(\mathrm{IPr})](100 \mathrm{mg}, 1.0$ equiv.), 1,2,4-triazole (1.1 equiv.), and THF $\left(2 \mathrm{~mL}\right.$ ). The reaction mixture was stirred at $25^{\circ} \mathrm{C}$ for $4 \mathrm{~h}$, filtered through Celite which was washed (THF, $3 \mathrm{x}$ $1 \mathrm{~mL}$ ). The organic phases were combined and the mixture was concentrated in vacuo until formation of a precipitate. Hexane $(5 \mathrm{~mL})$ was added. After decantation, the supernatant solution was removed, and the precipitate was washed with hexane $(2 \times 1 \mathrm{~mL})$ and dried in vacuo affording $2 \mathbf{2 a}(101 \mathrm{mg}, 91 \%)$.

\section{From $[\mathrm{Cu}(\mathrm{Cl})(\mathrm{IPr})]$}

In a glovebox, a screw capped vial was charged with $[\mathrm{Cu}(\mathrm{Cl})(\mathrm{NHC})](100 \mathrm{mg}, 1.0$ equiv.), 1,2,4-triazole (1.1 equiv.), $\mathrm{CsOH}\left(2.0\right.$ equiv.) and solvent $(2 \mathrm{~mL})$. The reaction was stirred at $25{ }^{\circ} \mathrm{C}$ for $4 \mathrm{~h}$. The reaction mixture was filtered through Celite, which was washed (reaction solvent, $3 \times 1 \mathrm{~mL}$ ). The organic phases were combined and the mixture was concentrated in vacuo until formation of a precipitate. Hexane $(5 \mathrm{~mL})$ was added. After decantation, the supernatant solution was removed, and the precipitate was washed with hexane $(2 \times 1 \mathrm{~mL})$ and dried in vacuo.

Table S1. Synthesis of copper dimers bearing different NHC ligands ${ }^{[a]}$

$$
[\mathrm{Cu}(\mathrm{Cl})(\mathrm{NHC})]+\stackrel{\mathrm{CsOH}(2 \text { equiv. })}{\text { solvent }(2 \mathrm{~mL}), 25^{\circ} \mathrm{C}, 4 \mathrm{~h}}
$$

\begin{tabular}{ccccc}
\hline Entry & {$[\mathrm{Cu}(\mathrm{Cl})(\mathrm{NHC})]$} & {$[\mathrm{Cu}(\operatorname{trz})(\mathrm{NHC})]_{2}$} & Solvent & Yield (\%) \\
\hline 1 & {$[\mathrm{Cu}(\mathrm{Cl})(\mathrm{IPr})]$} & {$[\mathrm{Cu}(\mathrm{trz})(\mathrm{IPr})]_{2}(\mathbf{2 a})$} & THF & 90 \\
2 & {$[\mathrm{Cu}(\mathrm{Cl})(\mathrm{IMes})]$} & {$[\mathrm{Cu}(\mathrm{trz})(\mathrm{IMes})]_{2}(\mathbf{2 b})$} & THF & 93 \\
3 & {$[\mathrm{Cu}(\mathrm{Cl})(\mathrm{SIMes})]$} & {$[\mathrm{Cu}(\operatorname{trz})(\mathrm{SIMes})]_{2}(\mathbf{2 c})$} & $\mathrm{CH}_{2} \mathrm{Cl}_{2}$ & 92 \\
4 & {$\left[\mathrm{Cu}(\mathrm{Cl})\left(\mathrm{I}^{t} \mathrm{Bu}\right)\right]$} & {$\left[\mathrm{Cu}(\operatorname{trz})\left({ }^{t} \mathrm{Bu}\right)\right]_{2}(\mathbf{2 d})$} & $\mathrm{CH}_{2} \mathrm{Cl}_{2}$ & 91 \\
5 & {$[\mathrm{Cu}(\mathrm{Cl})(\mathrm{ICy})]$} & {$[\mathrm{Cu}(\operatorname{trz})(\mathrm{ICy})]_{2}(\mathbf{2 e})$} & $\mathrm{CH}_{2} \mathrm{Cl}_{2}$ & 89
\end{tabular}

\footnotetext{
${ }^{[a]}$ Reaction conditions: [Cu(Cl)(NHC)] (100 mg, 1.0 equiv.), 1,2,4-triazole (1.1 equiv.), $\mathrm{CsOH}(2.0$ equiv.), solvent (2 $\mathrm{mL}), 25^{\circ} \mathrm{C}, 4 \mathrm{~h}$, under argon. ${ }^{[\mathrm{b}]}$ Isolated yield.
} 


\section{Synthesis of $[\mathrm{Cu}(\operatorname{trz})(\mathrm{NHC})]_{2}$ on large scale}

In a glovebox, a 50-mL round-bottomed flask was charged with $[\mathrm{Cu}(\mathrm{Cl})(\mathrm{NHC})](1 \mathrm{~g}, 1.0$ equiv.), 1,2,4-triazole (1.1 equiv.), $\mathrm{CsOH}$ (2.0 equiv.) and solvent $(20 \mathrm{~mL})$. The reaction was stirred at $25{ }^{\circ} \mathrm{C}$ for $4 \mathrm{~h}$. The solution was filtered through Celite which was washed (reaction solvent, $3 \times 5 \mathrm{~mL}$ ). The organic phases were combined and the mixture was concentrated in vacuo until formation of a precipitate. Hexane $(20 \mathrm{~mL})$ was added. The precipitate was collected by filtration, washed with hexane $(2 \times 5 \mathrm{~mL})$ and dried in vacuo.

Table S2. Synthesis of copper dimers bearing different NHC ligands on gram scale ${ }^{[a]}$

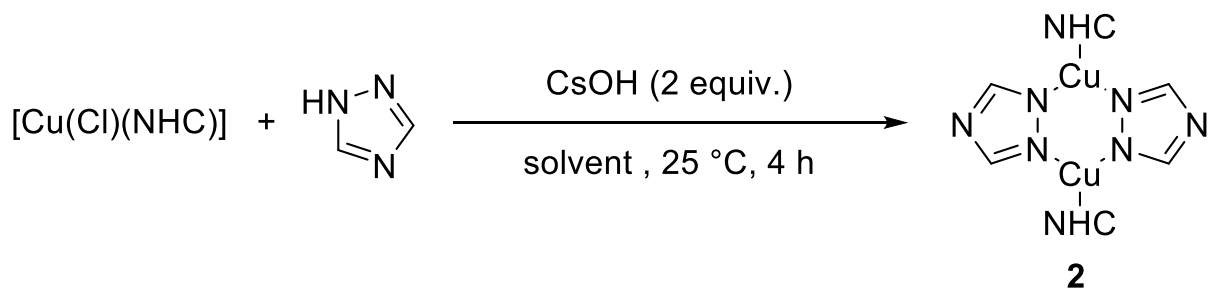

\begin{tabular}{ccccc}
\hline Entry & {$[\mathrm{Cu}(\mathrm{Cl})(\mathrm{NHC})]$} & {$[\mathrm{Cu}(\operatorname{trz})(\mathrm{NHC})]_{2}$} & Solvent & ${\text { Yield }(\%)^{[b]}}^{[}$ \\
\hline 1 & {$[\mathrm{Cu}(\mathrm{Cl})(\mathrm{IPr})]$} & $\mathbf{2 a}$ & THF & 92 \\
2 & {$[\mathrm{Cu}(\mathrm{Cl})(\mathrm{IMes})]$} & $\mathbf{2 b}$ & THF & 97 \\
3 & {$[\mathrm{Cu}(\mathrm{Cl})(\mathrm{SIMes})]$} & $\mathbf{2 c}$ & $\mathrm{CH}_{2} \mathrm{Cl}_{2}$ & 95
\end{tabular}

[a] Reaction conditions: $[\mathrm{Cu}(\mathrm{Cl})(\mathrm{NHC})](1 \mathrm{~g}, 1.0$ equiv.), 1,2,4-triazole (1.1 equiv.), $\mathrm{CsOH}$ (2.0 equiv.), solvent (20 $\mathrm{mL}), 25^{\circ} \mathrm{C}, 4 \mathrm{~h}$, under argon. [b] Isolated yield. 


\section{Characterisation of $[\mathrm{Cu}(\operatorname{trz})(\mathrm{NHC})]_{2}$ complexes}

Synthesis of bis-\{[N,N'-bis(2,6-(di-isopropyl)phenyl)imidazol-2-ylidene](1H-1,2,4-triazol-1-yl)\}copper (I), $[\mathrm{Cu}(\operatorname{trz})(\mathrm{IPr})]_{2}, 2 \mathrm{a}$

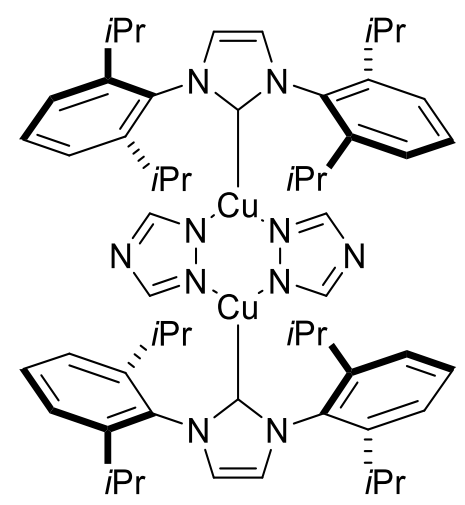

Reaction between $[\mathrm{Cu}(\mathrm{Cl})(\mathrm{IPr})](100 \mathrm{mg}, 0.21 \mathrm{mmol}), 1,2,4$-triazole $(16.0 \mathrm{mg}, 0.23 \mathrm{mmol})$ and $\mathrm{CsOH}(63.0 \mathrm{mg}, 0.42$ $\mathrm{mmol})$ in THF ( $2 \mathrm{~mL}$ ) led to the isolation of $\mathbf{2 a}$ as a colourless solid in $90 \%$ isolated yield (96 $\mathrm{mg})$

${ }^{1} \mathbf{H}$ NMR (500 MHz, $\left.\mathbf{C}_{\mathbf{6}} \mathbf{D}_{\mathbf{6}}, \mathbf{2 9 8 K}\right): \delta(\mathrm{ppm})=7.24\left(\mathrm{t},{ }^{3} J_{\mathrm{H}-\mathrm{H}}=8.0 \mathrm{~Hz}, 4 \mathrm{H}, \mathrm{C}_{\mathrm{Ar}} \mathrm{H}\right), 7.05\left(\mathrm{~d},{ }^{3} J_{\mathrm{H}-\mathrm{H}}=8.0 \mathrm{~Hz}, 8 \mathrm{H}, \mathrm{C}_{\mathrm{Ar}} \mathrm{H}\right)$, $6.73\left(\mathrm{~s}, 4 \mathrm{H}, \mathrm{CH}\right.$ trz), $6.39\left(\mathrm{~s}, 4 \mathrm{H}, \mathrm{H}^{4}\right.$ and $\mathrm{H}^{5}$ carbene), $2.83\left(\mathrm{sept},{ }^{3} J_{\mathrm{H}-\mathrm{H}}=6.8 \mathrm{~Hz}, 8 \mathrm{H}, \mathrm{CH}\left(\mathrm{CH}_{3}\right)_{2}\right), 1.13\left(\mathrm{~d},{ }^{3} J_{\mathrm{H}-\mathrm{H}}=6.8 \mathrm{~Hz}\right.$, $\left.24 \mathrm{H}, \mathrm{CH}\left(\mathrm{CH}_{3}\right)_{2}\right), 1.04\left(\mathrm{~d},{ }^{3} \mathrm{~J}_{\mathrm{H}-\mathrm{H}}=6.8 \mathrm{~Hz}, 24 \mathrm{H}, \mathrm{CH}\left(\mathrm{CH}_{3}\right)_{2}\right)$.

${ }^{13} \mathbf{C}-\left\{{ }^{1} \mathbf{H}\right\}$ NMR (126 MHz, $\left.\mathbf{C}_{\mathbf{6}} \mathbf{D}_{\mathbf{6}}, \mathbf{2 9 8 K}\right): \delta(\mathrm{ppm})=189.2\left(\mathrm{~s}, \mathrm{C}^{2}\right.$ carbene), $152.1\left(\mathrm{~s}, \mathrm{CH}\right.$ trz), $146.4\left(\mathrm{~s}, \mathrm{C}^{\mathrm{IV}}\right), 137.4(\mathrm{~s}$,

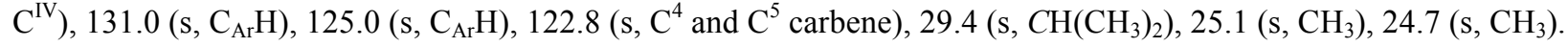

Anal. Calcd for $\mathrm{C}_{58} \mathrm{H}_{76} \mathrm{Cu}_{2} \mathrm{~N}_{10}$ : C, 66.96; H, 7.36; N, 13.45. Found: C, 66.77; H, 7.24; N, 13.33 .

Synthesis of bis-\{[N,N'-bis(2,4,6-(trimethyl)phenyl)imidazol-2-ylidene $](1 H-1,2,4$-triazol-1-yl) $\}$ copper $[\mathrm{Cu}(\mathrm{trz})(\mathrm{IMes})]_{2}, 2 \mathrm{~b}$

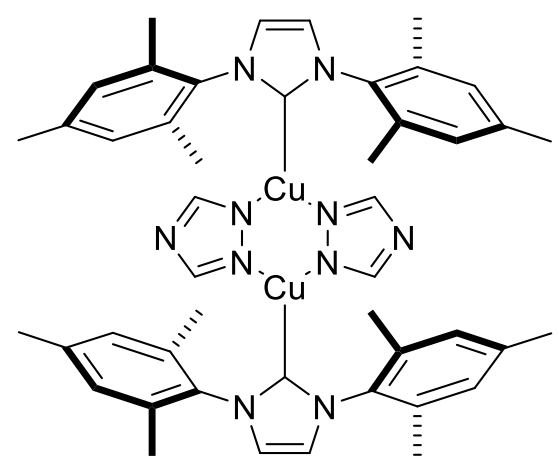

Reaction between $[\mathrm{Cu}(\mathrm{Cl})(\mathrm{IMes})](100 \mathrm{mg}, 0.25 \mathrm{mmol}), 1,2,4$-triazole $(18.8 \mathrm{mg}, 0.27 \mathrm{mmol})$ and $\mathrm{CsOH}(74.3 \mathrm{mg}, 0.42$ $\mathrm{mmol})$ in THF $(2 \mathrm{~mL})$ led to the isolation of $\mathbf{2} \mathbf{b}$ as a colourless solid in $93 \%$ isolated yield $(101 \mathrm{mg})$

${ }^{1} \mathbf{H}$ NMR (500 MHz, $\left.\mathbf{C D}_{2} \mathbf{C l}_{2}, \mathbf{2 9 8 K}\right): \delta(\mathrm{ppm})=7.33\left(\mathrm{~s}, 4 \mathrm{H}, \mathrm{CH}\right.$ trz), $6.98\left(\mathrm{~s}, 4 \mathrm{H}, \mathrm{H}^{4}\right.$ and $\mathrm{H}^{5}$ carbene), $6.90(\mathrm{~s}, 8 \mathrm{H}$, $\left.\mathrm{C}_{\mathrm{Ar}} \mathrm{H}\right), 2.24\left(\mathrm{~s}, 12 \mathrm{H}, \mathrm{CH}_{3}\right), 2.11\left(\mathrm{~s}, 24 \mathrm{H}, \mathrm{CH}_{3}\right)$.

${ }^{13} \mathrm{C}-\left\{{ }^{1} \mathrm{H}\right\}$ NMR (126 MHz, $\left.\mathbf{C D}_{2} \mathbf{C l}_{2}, \mathbf{2 9 8 K}\right): \delta(\mathrm{ppm})=185.5\left(\mathrm{~s}, \mathrm{C}^{2}\right.$ carbene), $151.5\left(\mathrm{~s}, \mathrm{CH}\right.$ trz), $138.8\left(\mathrm{~s}, \mathrm{C}^{\mathrm{IV}}\right), 136.2(\mathrm{~s}$, $\left.\mathrm{C}^{\mathrm{IV}}\right), 134.8\left(\mathrm{~s}, \mathrm{C}^{\mathrm{IV}}\right), 129.3\left(\mathrm{~s}, \mathrm{C}_{\mathrm{Ar}} \mathrm{H}\right), 121.4\left(\mathrm{~s}, \mathrm{C}^{4}\right.$ and $\mathrm{C}^{5}$ carbene $), 20.9\left(\mathrm{~s}, \mathrm{CH}_{3}\right), 17.7\left(\mathrm{~s}, \mathrm{CH}_{3}\right)$.

Anal. Calcd for $\mathrm{C}_{46} \mathrm{H}_{52} \mathrm{Cu}_{2} \mathrm{~N}_{10}$ : C, 63.35; H, 6.01; N, 16.06. Found: C, 63.28; H, 6.15; N, 15.97 . 
Synthesis of Bis-\{[N,N-Bis(2,4,6-(trimethyl)phenyl)imidazolidin-2-ylidene](1H-1,2,4-triazol-1-yl)\}copper (I), $[\mathrm{Cu}(\mathrm{trz})(\mathrm{SIMes})]_{2}, 2 \mathrm{c}$

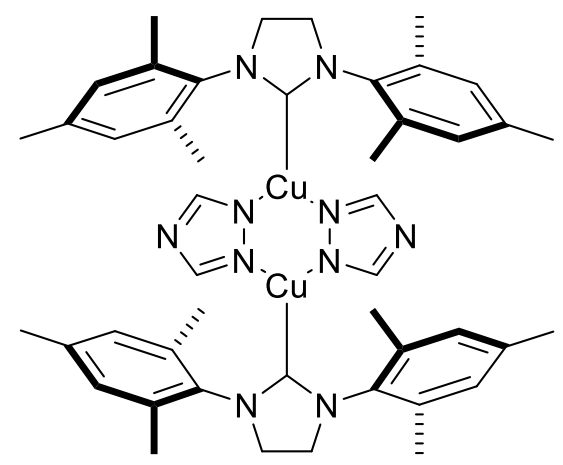

Reaction between [Cu(Cl)(SIMes)] $(100 \mathrm{mg}, 0.25 \mathrm{mmol}), 1,2,4$-triazole $(18.8 \mathrm{mg}, 0.27 \mathrm{mmol})$ and $\mathrm{CsOH}(74.3 \mathrm{mg}$, $0.42 \mathrm{mmol})$ in $\mathrm{CH}_{2} \mathrm{Cl}_{2}(2 \mathrm{~mL})$ led to the isolation of $2 \mathbf{c}$ as a colourless solid in $92 \%$ isolated yield $(101 \mathrm{mg})$

${ }^{1} \mathbf{H}$ NMR (500 MHz, $\left.\mathbf{C D}_{2} \mathbf{C l}_{2}, \mathbf{2 9 8 K}\right): \delta(\mathrm{ppm})=7.52\left(\mathrm{~s}, 4 \mathrm{H}, \mathrm{CH}\right.$ trz), $6.88\left(\mathrm{~s}, 8 \mathrm{H}, \mathrm{C}_{\mathrm{Ar}} \mathrm{H}\right), 3.89\left(\mathrm{~s}, 8 \mathrm{H}, \mathrm{H}^{4}\right.$ and $\mathrm{H}^{5}$ carbene), $2.35\left(\mathrm{~s}, 24 \mathrm{H}, \mathrm{CH}_{3}\right), 2.23\left(\mathrm{~s}, 12 \mathrm{H}, \mathrm{CH}_{3}\right)$.

${ }^{13} \mathrm{C}-\left\{{ }^{1} \mathbf{H}\right\}$ NMR (126 MHz, $\left.\mathbf{C D}_{2} \mathbf{C l}_{\mathbf{2}}, \mathbf{2 9 8 K}\right): \delta(\mathrm{ppm})=207.3\left(\mathrm{~s}, \mathrm{C}^{2}\right.$ carbene), $151.7\left(\mathrm{~s}, \mathrm{CH}\right.$ trz), $137.9\left(\mathrm{~s}, \mathrm{C}^{\mathrm{IV}}\right), 136.3(\mathrm{~s}$, $\left.\mathrm{C}^{\mathrm{IV}}\right), 135.6\left(\mathrm{~s}, \mathrm{C}^{\mathrm{IV}}\right), 129.5\left(\mathrm{~s}, \mathrm{C}_{\mathrm{Ar}} \mathrm{H}\right), 50.9\left(\mathrm{~s}, \mathrm{C}^{4}\right.$ and $\mathrm{C}^{5}$ carbene $), 20.9\left(\mathrm{~s}, \mathrm{CH}_{3}\right), 17.8\left(\mathrm{~s}, \mathrm{CH}_{3}\right)$.

Anal. Calcd for $\mathrm{C}_{46} \mathrm{H}_{56} \mathrm{Cu}_{2} \mathrm{~N}_{10}$ : C, 63.06; H, 6.44; N, 15.99. Found: C, 63.12; H, 6.47; N, 15.86 .

Synthesis of Bis- $\left\{\left[N, N^{\prime}\right.\right.$-(di-tert-butyl)imidazol-2-ylidene $\left.](1 H-1,2,4-t r i a z o l-1-y l)\right\} \operatorname{copper}(\mathrm{I}),\left[\mathrm{Cu}(\operatorname{trz})\left(\mathrm{I}^{t} \mathrm{Bu}\right)\right]_{2}, 2 \mathrm{~d}$<smiles>CC1N(C(C)(C)C)C=CN1C(C)(C)C</smiles>

Reaction between $\left[\mathrm{Cu}(\mathrm{Cl})\left(\mathrm{I}^{t} \mathrm{Bu}\right)\right](100 \mathrm{mg}, 0.36 \mathrm{mmol}), 1,2,4$-triazole $(27.2 \mathrm{mg}, 0.39 \mathrm{mmol})$ and $\mathrm{CsOH}(107 \mathrm{mg}, 0.72$ $\mathrm{mmol})$ in $\mathrm{CH}_{2} \mathrm{Cl}_{2}(2 \mathrm{~mL})$ led to the isolation of $\mathbf{2 d}$ as a colourless solid in $91 \%$ isolated yield $(102 \mathrm{mg})$.

${ }^{1} \mathbf{H}$ NMR (500 MHz, $\left.\mathbf{C D}_{\mathbf{2}} \mathbf{C l}_{2}, \mathbf{2 9 8 K}\right): \delta(\mathrm{ppm})=7.85\left(\mathrm{~s}, 4 \mathrm{H}, \mathrm{CH}\right.$ trz), $7.10\left(\mathrm{~s}, 4 \mathrm{H}, \mathrm{H}^{4}\right.$ and $\mathrm{H}^{5}$ carbene), $1.76(\mathrm{~s}, 36 \mathrm{H}$, $\left.\mathrm{CH}_{3}\right)$.

${ }^{13} \mathrm{C}-\left\{{ }^{1} \mathrm{H}\right\}$ NMR (126 MHz, $\left.\mathbf{C D}_{2} \mathrm{Cl}_{2}, \mathbf{2 9 8 K}\right): \delta(\mathrm{ppm})=180.4\left(\mathrm{~s}, \mathrm{C}^{2}\right.$ carbene), 151.7 (s, CH trz), 115.5 (s, $\mathrm{C}^{4}$ and $\mathrm{C}^{5}$ carbene), $57.0\left(\mathrm{~s}, \mathrm{C}^{\mathrm{IV}}\right), 31.2\left(\mathrm{~s}, \mathrm{CH}_{3}\right)$.

Anal. Calcd for $\mathrm{C}_{26} \mathrm{H}_{44} \mathrm{Cu}_{2} \mathrm{~N}_{10}$ : C, 50.06; H, 7.11; N, 22.45. Found: C, 49.87; H, 7.18; N, 22.32 . 
<smiles>ClC1N(C2CCCCC2)C=CN1C1CCCCC1N1C=CN(C2CCCCC2)C1C1(Cl)N(C2CCCCC2)C=CN1C1CCCCC1</smiles>

Reaction between $[\mathrm{Cu}(\mathrm{Cl})(\mathrm{ICy})](100 \mathrm{mg}, 0.30 \mathrm{mmol})$, 1,2,4-triazole (22.9 mg, $0.33 \mathrm{mmol})$ and $\mathrm{CsOH}(90.5 \mathrm{mg}, 0.60$ $\mathrm{mmol})$ in $\mathrm{CH}_{2} \mathrm{Cl}_{2}(2 \mathrm{~mL})$ led to the isolation of $2 \mathbf{e}$ as a colourless solid in $89 \%$ isolated yield $(97.2 \mathrm{mg})$.

${ }^{1} \mathbf{H}$ NMR (500 MHz, $\left.\mathbf{C D}_{\mathbf{2}} \mathbf{C l}_{\mathbf{2}}, \mathbf{2 9 8 K}\right): \delta(\mathrm{ppm})=7.90\left(\mathrm{~s}, 4 \mathrm{H}, \mathrm{CH}\right.$ trz), $7.00\left(\mathrm{~s}, 4 \mathrm{H}, \mathrm{H}^{4}\right.$ and $\mathrm{H}^{5}$ carbene), $4.42\left(\mathrm{tt},{ }^{3} J_{\mathrm{HH}}=\right.$ $12.1 \mathrm{~Hz},{ }^{5} J_{\mathrm{HH}}=3.8 \mathrm{~Hz}, 4 \mathrm{H}, \mathrm{CH}$ cyclohexyl), 2.16-2.09 (m, 8H, cyclohexyl), 1.89-1.83 (m, 9H, cyclohexyl), 1.76-1.67 (m, 11H, cyclohexyl), 1.38-1.22 (m, 8H, cyclohexyl), 1.43-1.32 (m, 8H, cyclohexyl), 1.27-1.17 (m, 4H, cyclohexyl).

${ }^{13} \mathbf{C}-\left\{{ }^{1} \mathbf{H}\right\}$ NMR (126 MHz, $\left.\mathbf{C D}_{2} \mathbf{C l}_{2}, \mathbf{2 9 8 K}\right): \delta(\mathrm{ppm})=181.2\left(\mathrm{~s}, \mathrm{C}^{2}\right.$ carbene), $151.8\left(\mathrm{~s}, \mathrm{CH}\right.$ trz), $116.2\left(\mathrm{~s}, \mathrm{C}^{4}\right.$ and $\mathrm{C}^{5}$ carbene), 60.5 (s, CH cyclohexyl), $34.5\left(\mathrm{~s}, \mathrm{CH}_{2}\right), 25.6\left(\mathrm{~s}, \mathrm{CH}_{2}\right), 25.4\left(\mathrm{~s}, \mathrm{CH}_{2}\right)$.

Elemental analysis was attempted several times, but satisfactory data could not be obtained, presumably due to instability of the complex. 


\section{Crystallographic data for complexes 2a-e}

\section{$[\mathrm{Cu}(\operatorname{trz})(\operatorname{IPr})]_{2}, 2 \mathrm{a}$}

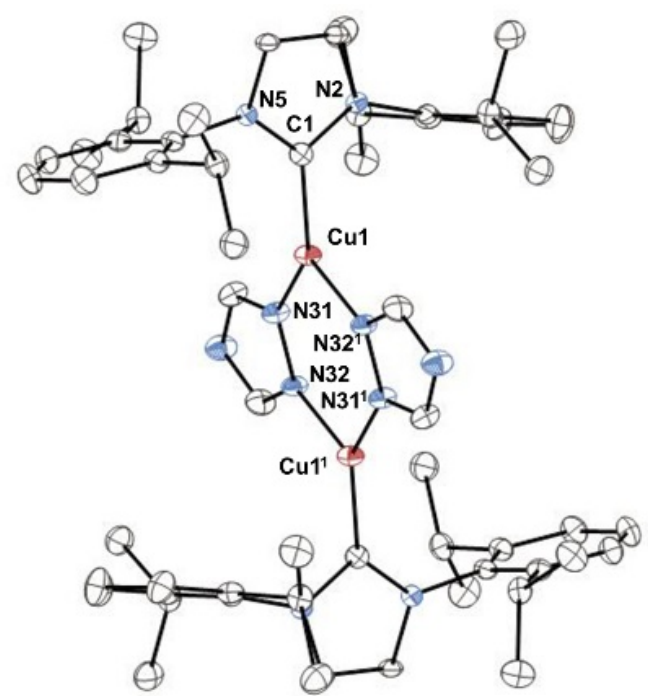

Figure S1. Molecular structure of 2a. All hydrogen atoms are omitted for clarity. Thermal ellipsoids are shown at the $50 \%$ probability level.

\begin{tabular}{|c|c|}
\hline Complex & $2 a$ \\
\hline CCDC number & 1442422 \\
\hline Emperical formula & $\mathrm{C}_{58} \mathrm{H}_{76} \mathrm{Cu}_{2} \mathrm{~N}_{10}$ \\
\hline Formula Weight & 520.20 \\
\hline Crystal color, Habit & colorless, prism \\
\hline Temperature (K) & 93.15 \\
\hline Crystal system & triclinic \\
\hline Space group & P-1 (\#2) \\
\hline Unit cell dim. & $0.100 \times 0.100 \times 0.100 \mathrm{~mm}$ \\
\hline Lattice type & Primitive \\
\hline $\begin{array}{l}\text { Lattice parameter } \\
\text { a,b,c }(\AA)\end{array}$ & $\begin{array}{l}a=10.175(4) \\
b=10.563(4) \\
c=13.979(5)\end{array}$ \\
\hline$\alpha, \beta, \gamma\left({ }^{\circ}\right)$ & $\begin{array}{l}\alpha=106.427(7) \\
\beta=98.050(10) \\
\gamma=105.329(5)\end{array}$ \\
\hline Volume $(\AA)^{3}$ & $1351.8(9)$ \\
\hline $\mathrm{Z}$ & 2 \\
\hline Density calculated & $1.278 \mathrm{~g} / \mathrm{cm}^{3}$ \\
\hline Absorption coefficient $\left(\mathrm{cm}^{-1}\right)$ & $8.338 \mathrm{~cm}^{-1}$ \\
\hline $\mathrm{F}(000)$ & 552.00 \\
\hline Diffractometer & Mercury70 \\
\hline Radiation & $\operatorname{MoK} \alpha(\lambda=0.71075 \AA)$ \\
\hline Voltage, Current & $50 \mathrm{kV}, 16 \mathrm{~mA}$ \\
\hline Theta range for data collection $\left({ }^{\circ}\right)$ & $2 \theta_{\max }=50.7^{\circ}$ \\
\hline
\end{tabular}




\begin{tabular}{|c|c|}
\hline & Total: 8028 \\
Reflexions collected & Unique: $4673\left(\mathrm{R}_{\text {int }}=0.0310\right)$ \\
\hline Correction & Lorentz-polarization \\
& Absorption \\
& (trans. factors: $0.596-0.920)$ \\
\hline Structure solution & Patterson Methods (DIRDIF99 PATTY) \\
\hline Refinement method & Full-matrix least-squares on $\mathrm{F}^{2}$ \\
\hline Anomalous dispersion & All non-hydrogen atoms \\
\hline No. Observations (all reflections) & 4673 \\
\hline No. variables & 324 \\
\hline Reflection/parameter ratio & 14.42 \\
\hline Goodness-of-fit on $\mathrm{F}^{2}$ & 1.050 \\
\hline Final R indices [I>2sigma(I)] & 0.0416 \\
\hline R indices (all data) & 0.0527 \\
\hline Maximum peak in Final Diff Map $\left(\mathrm{e} . \AA^{-3}\right)$ & $0.36 \mathrm{e}^{-} / \AA^{3}$ \\
\hline Minimum peak in Final Diff Map $\left(\mathrm{e} . \AA^{-3}\right)$ & $-0.34 \mathrm{e}^{-} / \AA^{3}$ \\
\hline Max shift/error in final cycle & 0.000 \\
\hline
\end{tabular}

Table S3. Selected bond distances, angles and torsion angles for $\mathbf{2 a}$

\begin{tabular}{|c|c|c|}
\hline \multicolumn{3}{|c|}{ Bond distances $(\AA)$} \\
\hline $\mathrm{Cu} 1-\mathrm{C} 1=1.896(2)$ & $\mathrm{Cu} 1-\mathrm{N} 32^{1}=1.964(2)$ & $\mathrm{Cu} 1-\mathrm{N} 31=2.001(2)$ \\
\hline \multicolumn{3}{|c|}{ Bond angles $\left(^{\circ}\right)$} \\
\hline $\mathrm{N} 32^{1}-\mathrm{Cu} 1-\mathrm{C} 1=134.57(10)$ & $\mathrm{N} 31-\mathrm{Cu} 1-\mathrm{C} 1=119.15(10)$ & $\mathrm{N} 31-\mathrm{Cu} 1-\mathrm{N} 32^{1}=106.17(9)$ \\
\hline \multicolumn{3}{|c|}{ Torsional angles $\left(^{\circ}\right)$} \\
\hline $\mathrm{N} 31-\mathrm{Cu} 1-\mathrm{C} 1-\mathrm{N} 2=-76.7(2)$ & $\mathrm{N} 32^{1}-\mathrm{Cu} 1-\mathrm{C} 1-\mathrm{N} 2=98.9(2)$ & $\mathrm{N} 31-\mathrm{Cu} 1-\mathrm{C} 1-\mathrm{N} 5=99.3(2)$ \\
\hline $\mathrm{N} 32^{1}-\mathrm{Cu} 1-\mathrm{C} 1-\mathrm{N} 5=-85.0(2)$ & $\mathrm{Cu} 1-\mathrm{N} 31-\mathrm{N} 32-\mathrm{Cu} 1^{1}=5.4(2)$ & $\mathrm{N} 31-\mathrm{Cu} 1-\mathrm{N} 32^{1}-\mathrm{N} 31^{1}=4.37(15)$ \\
\hline $\mathrm{N} 32^{1}-\mathrm{Cu} 1-\mathrm{N} 31-\mathrm{N} 32=-4.59(16)$ & $\mathrm{C} 1-\mathrm{Cu} 1-\mathrm{Cu} 1^{1}=\mathbf{1 7 0 . 2 2}(7)$ & \\
\hline
\end{tabular}


$[\mathrm{Cu}(\operatorname{trz})(\mathrm{IMes})]_{2}, \mathbf{2 b}$

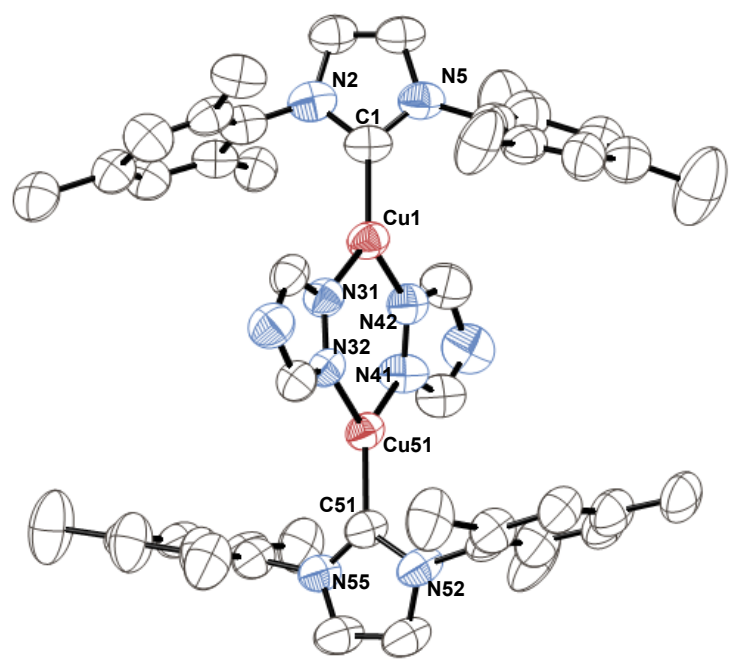

Figure S2. Molecular structure of 2b. All hydrogen atoms are omitted for clarity. Thermal ellipsoids are shown at the $50 \%$ probability level.

\begin{tabular}{|c|c|}
\hline Complex & $2 \mathbf{b}$ \\
\hline CCDC number & 1442423 \\
\hline Emperical formula & $\mathrm{C}_{46} \mathrm{H}_{52} \mathrm{Cu}_{2} \mathrm{~N}_{10}$ \\
\hline Formula Weight & 872.08 \\
\hline Crystal color, Habit & colorless, prism \\
\hline Temperature $(\mathrm{K})$ & 173.15 \\
\hline Crystal system & orthorhombic \\
\hline Space group & $\mathrm{P} 2{ }_{1}{ }_{1}{ }^{2}{ }_{1}(\# 19)$ \\
\hline Unit cell dim. & $0.150 \times 0.120 \times 0.080 \mathrm{~mm}$ \\
\hline Lattice type & Primitive \\
\hline $\begin{array}{l}\text { Lattice parameter } \\
\qquad \mathrm{a}, \mathrm{b}, \mathrm{c}(\AA)\end{array}$ & $\begin{array}{l}\mathrm{a}=15.431(5) \\
\mathrm{b}=16.964(5) \\
\mathrm{c}=23.348(7)\end{array}$ \\
\hline Volume $(\AA)^{3}$ & $6112(3)$ \\
\hline $\mathrm{Z}$ & 4 \\
\hline Density calculated & $0.948 \mathrm{~g} / \mathrm{cm}^{3}$ \\
\hline Absorption coefficient $\left(\mathrm{cm}^{-1}\right)$ & $7.277 \mathrm{~cm}^{-1}$ \\
\hline $\mathrm{F}(000)$ & 1824.00 \\
\hline Diffractometer & XtaLAB P200 \\
\hline Radiation & $\begin{array}{c}\text { MoKa }(1=0.71075 \AA) \\
\text { multi-layer mirror monochromated }\end{array}$ \\
\hline Voltage, Current & $45 \mathrm{kV}, 66 \mathrm{~mA}$ \\
\hline Theta range for data collection $\left(^{\circ}\right)$ & $2 \mathrm{q}_{\max }=50.7^{0}$ \\
\hline Reflexions collected & $\begin{array}{c}\text { Total: } 48979 \\
\text { Unique: } 11132\left(\mathrm{R}_{\mathrm{int}}=0.1784\right)\end{array}$ \\
\hline
\end{tabular}




\begin{tabular}{|c|c|}
\hline & Parsons quotients (Flack x parameter): 1499 \\
\hline Correction & Lorentz-polarization \\
& Absorption \\
& (trans. factors: $0.617-0.943)$ \\
\hline Structure solution & Direct Methods (SIR2004) \\
\hline Refinement method & Full-matrix least-squares on $\mathrm{F}^{2}$ \\
\hline Anomalous dispersion & All non-hydrogen atoms \\
\hline No. Observations (all reflections) & 11132 \\
\hline No. variables & 534 \\
\hline Reflection/parameter ratio & 20.85 \\
\hline Goodness-of-fit on $\mathrm{F}^{2}$ & 1.354 \\
\hline Final R indices [I 2 sigma(I) & 0.1642 \\
\hline R indices (all data) & 0.2579 \\
\hline Maximum peak in Final Diff Map $\left(\mathrm{e} . \AA^{-3}\right)$ & $2.46 \mathrm{e}^{-} / \AA^{3}$ \\
\hline Minimum peak in Final Diff Map $\left(\mathrm{e} . \AA^{-3}\right)$ & $-0.66 \mathrm{e}^{-} / \AA^{3}$ \\
\hline Max shift/error in final cycle & 0.001 \\
\hline
\end{tabular}

Table S4. Selected bond distances, angles and torsion angles for $\mathbf{2 b}$

\begin{tabular}{|c|c|c|}
\hline \multicolumn{3}{|c|}{ Bond distances $(\AA)$} \\
\hline $\mathrm{Cu} 1-\mathrm{C} 1=1.900(19)$ & $\mathrm{Cu} 1-\mathrm{N} 31=2.011(17)$ & Cu1-N42 = 1.927(17) \\
\hline $\mathrm{Cu} 51-\mathrm{C} 51=1.922(18)$ & $\mathrm{Cu} 51-\mathrm{N} 41=1.992(19)$ & Cu1-N42 $=1.927(17)$ \\
\hline \multicolumn{3}{|c|}{ Bond angles $\left({ }^{\circ}\right)$} \\
\hline $\mathrm{N} 31-\mathrm{Cu} 1-\mathrm{N} 42=105.6(7)$ & $\mathrm{N} 42-\mathrm{Cu} 1-\mathrm{C} 1=126.4(8)$ & $\mathrm{N} 31-\mathrm{Cu} 1-\mathrm{C} 1=128.0(8)$ \\
\hline N32-Cu51-N41 = 105.8(7) & N32-Cu51-C51 = 127.7(8) & N41-Cu51-C51 = 126.5(8) \\
\hline \multicolumn{3}{|c|}{ Torsional angles $\left({ }^{\circ}\right)$} \\
\hline $\mathrm{N} 31-\mathrm{Cu} 1-\mathrm{C} 1-\mathrm{N} 2=-59(2)$ & N42-Cu1-C1-N2 = 118.2(15) & N31-Cu1-C1-N5 = 123.4(15) \\
\hline N42-Cu1-C1-N5 = -60(2) & N31-Cu1-N42-N41 = 4.9(15) & N42-Cu1-N31-N32 = 4.8(14) \\
\hline N32-Cu51-C51-N52 = -98.1(15) & $\mathrm{N} 41-\mathrm{Cu} 51-\mathrm{C} 51-\mathrm{N} 52=81.9(18)$ & N32-Cu51-C51-N55 = 83(2) \\
\hline $\mathrm{N} 41-\mathrm{Cu} 51-\mathrm{C} 51-\mathrm{N} 55=-97.3(17)$ & N32-Cu51-N41-N42 = 6.3(16) & N41-Cu51-N32-N31 = 3.1(14) \\
\hline Cu1-N31-N32-Cu51 = -8(2) & Cu51-N41-N42-Cu1 = -10(3) & C1-Cu1-Cu51 = 178.0(7) \\
\hline
\end{tabular}

The crystals of $\mathbf{2 b}$ showed very weak diffraction at higher angles, leading to an elevated value of Rint. Following structure determination, this was found to be due to channels running along the crystallographic $b$-axis, filled with highly disordered solvent molecules, occupying approximately $37 \%$ of the volume of the unit cell (2235 $\AA$ of $6112 \AA$ ). No chemically sensible solvent model could be made from the electron density peaks, which leads to elevated values of $\mathrm{R} 1$ and $w R 2$. 
$[\mathrm{Cu}(\mathrm{trz})(\mathrm{SIMes})]_{2}, \mathbf{2 c}$

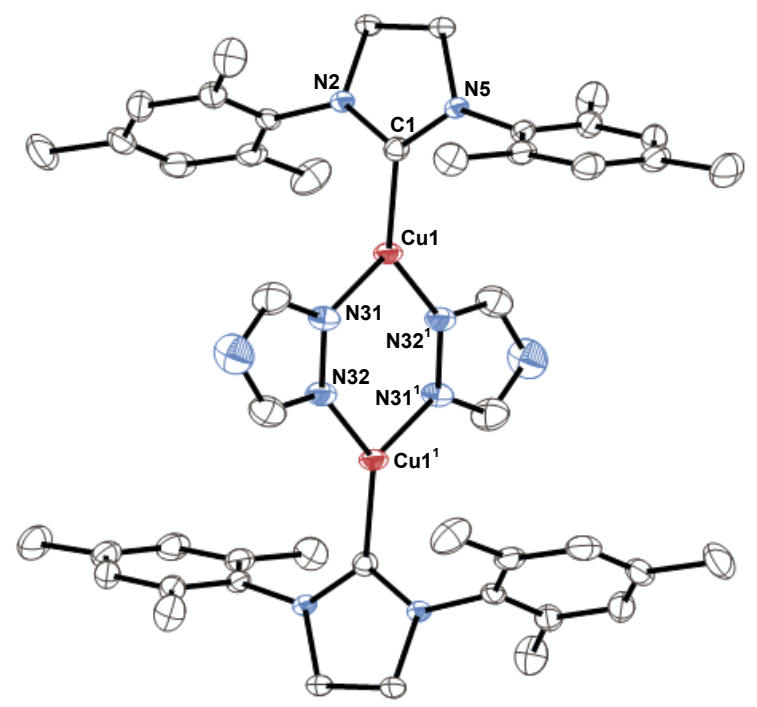

Figure S3. Molecular structure of 2c. All hydrogen atoms are omitted for clarity. Thermal ellipsoids are shown at the $50 \%$ probability level.

\begin{tabular}{|c|c|}
\hline Complex & $2 c$ \\
\hline CCDC number & 1442424 \\
\hline Emperical formula & $\mathrm{C}_{46} \mathrm{H}_{56} \mathrm{Cu}_{2} \mathrm{~N}_{10}$ \\
\hline Formula Weight & 876.11 \\
\hline Crystal color, Habit & colorless, prism \\
\hline Temperature $(\mathrm{K})$ & 173.15 \\
\hline Crystal system & monoclinic \\
\hline Space group & $\mathrm{P} 2{ }_{1} / \mathrm{c}(\# 14)$ \\
\hline Unit cell dim. & $0.150 \times 0.120 \times 0.060 \mathrm{~mm}$ \\
\hline Lattice type & Primitive \\
\hline $\begin{array}{l}\text { Lattice parameter } \\
\qquad \text { a,b,c }(\AA)\end{array}$ & $\begin{array}{c}\mathrm{a}=11.3017(15) \\
\mathrm{b}=13.5088(15) \\
\mathrm{c}=14.966(2)\end{array}$ \\
\hline$\alpha, \beta, \gamma\left(^{\circ}\right)$ & $\beta=108.686(4)$ \\
\hline Volume $(\AA)^{3}$ & $\mathrm{~V}=2164.5(5)$ \\
\hline $\mathrm{Z}$ & 2 \\
\hline Density calculated & $1.344 \mathrm{~g} / \mathrm{cm}^{3}$ \\
\hline Absorption coefficient $\left(\mathrm{cm}^{-1}\right)$ & $10.276 \mathrm{~cm}^{-1}$ \\
\hline $\mathrm{F}(000)$ & 920.00 \\
\hline Diffractometer & XtaLAB P200 \\
\hline Radiation & $\begin{array}{c}\text { MoK } \alpha(\lambda=0.71075 \AA) \\
\text { multi-layer mirror monochromated }\end{array}$ \\
\hline Voltage, Current & $45 \mathrm{kV}, 66 \mathrm{~mA}$ \\
\hline Theta range for data collection $\left(^{\circ}\right)$ & $2 \theta_{\max }=50.7^{\circ}$ \\
\hline Reflexions collected & Total: 25768 \\
\hline
\end{tabular}




\begin{tabular}{|c|c|}
\hline & Unique: $3935\left(\mathrm{R}_{\text {int }}=0.0379\right)$ \\
\hline Correction & Lorentz-polarization \\
& Absorption \\
& (trans. factors: $0.852-0.940)$ \\
\hline Structure solution & Direct Methods (SIR2011) \\
\hline Refinement method & Full-matrix least-squares on $\mathrm{F}^{2}$ \\
\hline Anomalous dispersion & All non-hydrogen atoms \\
\hline No. Observations (all reflections) & 3935 \\
\hline No. variables & 268 \\
\hline Reflection/parameter ratio & 14.68 \\
\hline Goodness-of-fit on $\mathrm{F}^{2}$ & 1.036 \\
\hline Final R indices [I 2 sigma(I)] & 0.0315 \\
\hline R indices (all data) & 0.0422 \\
\hline Maximum peak in Final Diff Map $\left(\mathrm{e} . \AA^{-3}\right)$ & $0.30 \mathrm{e}^{-} / \AA^{3}$ \\
\hline Minimum peak in Final Diff Map $\left(\mathrm{e} . \AA^{-3}\right)$ & $-0.27 \mathrm{e}^{-} / \AA^{3}$ \\
\hline Max shift/error in final cycle & 0.002 \\
\hline
\end{tabular}

Table S5. Selected bond distances, angles and torsion angles for $\mathbf{2 c}$

\begin{tabular}{|c|c|c|}
\hline \multicolumn{3}{|c|}{ Bond distances $(\AA)$} \\
\hline $\mathrm{Cu} 1-\mathrm{C} 1=1.900(2)$ & $\mathrm{Cu} 1-\mathrm{N} 32^{1}=1.9676(2)$ & $\mathrm{Cu} 1-\mathrm{N} 31=1.9924(19)$ \\
\hline \multicolumn{3}{|c|}{ Bond angles $\left(^{\circ}\right)$} \\
\hline $\mathrm{N} 32^{1}-\mathrm{Cu} 1-\mathrm{C} 1=129.76(8)$ & $\mathrm{N} 31-\mathrm{Cu} 1-\mathrm{C} 1=124.82(7)$ & $\mathrm{N} 31-\mathrm{Cu} 1-\mathrm{N} 32^{1}=105.29(7)$ \\
\hline \multicolumn{3}{|c|}{ Torsional angles $\left(^{\circ}\right)$} \\
\hline $\mathrm{N} 31-\mathrm{Cu} 1-\mathrm{C} 1-\mathrm{N} 2=61.6(2)$ & $\mathrm{N} 32^{1}-\mathrm{Cu} 1-\mathrm{C} 1-\mathrm{N} 2=-123.24(15)$ & $\mathrm{N} 31-\mathrm{Cu} 1-\mathrm{C} 1-\mathrm{N} 5=-111.98(15)$ \\
\hline $\mathrm{N} 32^{1}-\mathrm{Cu} 1-\mathrm{C} 1-\mathrm{N} 5=63.2(2)$ & $\mathrm{Cu} 1-\mathrm{N} 31-\mathrm{N} 32-\mathrm{Cu} 1^{1}=-7.1(3)$ & $\mathrm{N} 31-\mathrm{Cu} 1-\mathrm{N} 32^{1}-\mathrm{N} 31^{1}=-5.94(19)$ \\
\hline $\mathrm{N} 32^{1}-\mathrm{Cu} 1-\mathrm{N} 31-\mathrm{N} 32=-5.85(18)$ & $\mathrm{C} 1-\mathrm{Cu} 1-\mathrm{Cu} 1^{1}=\mathbf{1 7 7 . 0 5 ( 6 )}$ & \\
\hline
\end{tabular}


$\left[\mathrm{Cu}(\operatorname{trz})\left(\mathrm{I}^{t} \mathrm{Bu}\right)\right]_{2}, \mathbf{2 d}$

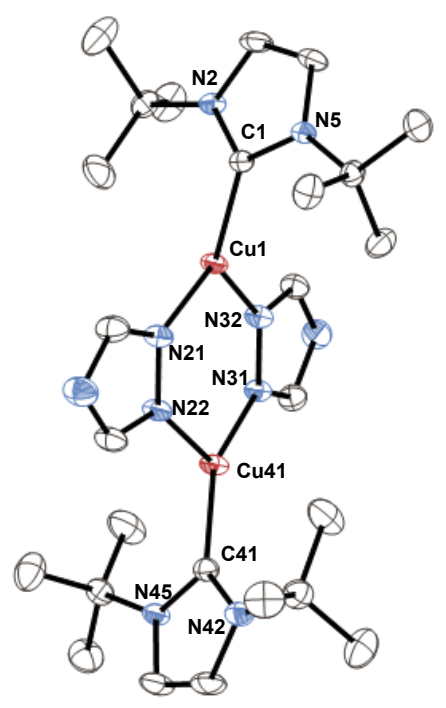

Figure S4. Molecular structure of 2d. All hydrogen atoms are omitted for clarity. Thermal ellipsoids are shown at the $50 \%$ probability level.

\begin{tabular}{|c|c|}
\hline Complex & $2 d$ \\
\hline CCDC number & 1442425 \\
\hline Emperical formula & $\mathrm{C}_{26} \mathrm{H}_{44} \mathrm{Cu}_{2} \mathrm{~N}_{10}$ \\
\hline Formula Weight & 623.79 \\
\hline Crystal color, Habit & colorless, prism \\
\hline Temperature $(\mathrm{K})$ & 173.15 \\
\hline Crystal system & monoclinic \\
\hline Space group & $\mathrm{P} 2{ }_{1} / \mathrm{c}(\# 14)$ \\
\hline Unit cell dim. & $0.210 \times 0.150 \times 0.050 \mathrm{~mm}$ \\
\hline Lattice type & Primitive \\
\hline $\begin{array}{l}\text { Lattice parameter } \\
\text { a,b,c }(\AA)\end{array}$ & $\begin{array}{c}\mathrm{a}=11.1337(10) \\
\mathrm{b}=10.4263(9) \\
\mathrm{c}=25.690(2)\end{array}$ \\
\hline$\alpha, \beta, \gamma\left({ }^{\circ}\right)$ & $\beta=92.586(3)$ \\
\hline Volume $(\AA)^{3}$ & $\mathrm{~V}=2979.1(4)$ \\
\hline $\mathrm{Z}$ & 4 \\
\hline Density calculated & $1.391 \mathrm{~g} / \mathrm{cm}^{3}$ \\
\hline Absorption coefficient $\left(\mathrm{cm}^{-1}\right)$ & $14.614 \mathrm{~cm}^{-1}$ \\
\hline $\mathrm{F}(000)$ & 1312.00 \\
\hline Diffractometer & XtaLAB P200 \\
\hline Radiation & $\begin{array}{c}\operatorname{MoK} \alpha(\lambda=0.71075 \AA) \\
\text { multi-layer mirror monochromated }\end{array}$ \\
\hline Voltage, Current & $45 \mathrm{kV}, 66 \mathrm{~mA}$ \\
\hline Theta range for data collection $\left({ }^{\circ}\right)$ & $2 \theta_{\max }=50.7^{\circ}$ \\
\hline
\end{tabular}




\begin{tabular}{|c|c|}
\hline & Total: 35438 \\
Reflexions collected & Unique: 5453 ( $\left.\mathrm{R}_{\text {int }}=0.0196\right)$ \\
\hline Correction & Lorentz-polarization \\
& Absorption \\
& (trans. factors: 0.827 - 0.930) \\
\hline Structure solution & Direct Methods (SIR2011) \\
\hline Refinement method & Full-matrix least-squares on $\mathrm{F}^{2}$ \\
\hline Anomalous dispersion & All non-hydrogen atoms \\
\hline No. Observations (all reflections) & 5453 \\
\hline No. variables & 355 \\
\hline Reflection/parameter ratio & 15.36 \\
\hline Goodness-of-fit on $\mathrm{F}^{2}$ & 1.033 \\
\hline Final R indices [I>2sigma(I)] & 0.0203 \\
\hline R indices (all data) & 0.0224 \\
\hline Maximum peak in Final Diff Map $\left(\mathrm{e} . \AA^{-3}\right)$ & $0.26 \mathrm{e}^{-} / \AA^{3}$ \\
\hline Minimum peak in Final Diff Map $\left(\mathrm{e} . \AA^{-3}\right)$ & $-0.26 \mathrm{e}^{-} / \AA^{3}$ \\
\hline Max shift/error in final cycle & 0.002 \\
\hline
\end{tabular}

Table S6. Selected bond distances, angles and torsion angles for $\mathbf{2 d}$

\begin{tabular}{|c|c|c|}
\hline \multicolumn{3}{|c|}{ Bond distances $(\AA)$} \\
\hline $\mathrm{Cu} 1-\mathrm{C} 1=1.9246(13)$ & $\mathrm{Cu} 1-\mathrm{N} 21=1.9570(11)$ & $\mathrm{Cu} 1-\mathrm{N} 32=2.0528(13)$ \\
\hline $\mathrm{Cu} 41-\mathrm{C} 41=1.9204(14)$ & $\mathrm{Cu} 41-\mathrm{N} 31=1.9604(12)$ & $\mathrm{Cu} 1-\mathrm{N} 22=2.0272(13)$ \\
\hline \multicolumn{3}{|c|}{ Bond angles $\left({ }^{\circ}\right)$} \\
\hline $\mathrm{N} 21-\mathrm{Cu} 1-\mathrm{N} 32=107.13(5)$ & N32-Cu1-C1 = 111.18(5) & $\mathrm{N} 21-\mathrm{Cu} 1-\mathrm{C} 1=141.68(5)$ \\
\hline N22-Cu41-N31 = 107.06(5) & $\mathrm{N} 22-\mathrm{Cu} 41-\mathrm{C} 41=112.70(5)$ & N31-Cu41-C41 = 140.20(5) \\
\hline \multicolumn{3}{|c|}{ Torsional angles $\left(^{\circ}\right)$} \\
\hline N21-Cu1-C1-N2 = 81.21(11) & N32-Cu1-C1-N2 = 81.21(11) & N21-Cu1-C1-N5 = 91.18(13) \\
\hline N32-Cu1-C1-N5 = -87.18(10) & N21-Cu1-N32-N31 = -5.91(12) & N32-Cu1-N21-N22 = 5.02(11) \\
\hline $\mathrm{N} 22-\mathrm{Cu} 41-\mathrm{C} 41-\mathrm{N} 42=-74.31(12)$ & N31-Cu41-C41-N42 = 108.53(12) & N22-Cu41-C41-N45 = 92.75(11) \\
\hline $\mathrm{N} 31-\mathrm{Cu} 41-\mathrm{C} 41-\mathrm{N} 45=-84.41(13)$ & N22-Cu41-N31-N32 = 10.35(11) & $\mathrm{N} 31-\mathrm{Cu} 41-\mathrm{N} 22-\mathrm{N} 21=-11.69(12)$ \\
\hline Cu1-N21-N22-Cu41 = 3.99(18) & Cu41-N31-N32-Cu1 = -2.56(17) & C1-Cu1-Cu51 = 161.76(4) \\
\hline
\end{tabular}


$[\mathrm{Cu}(\operatorname{trz})(\mathrm{ICy})]_{2}, \mathbf{2 e}$

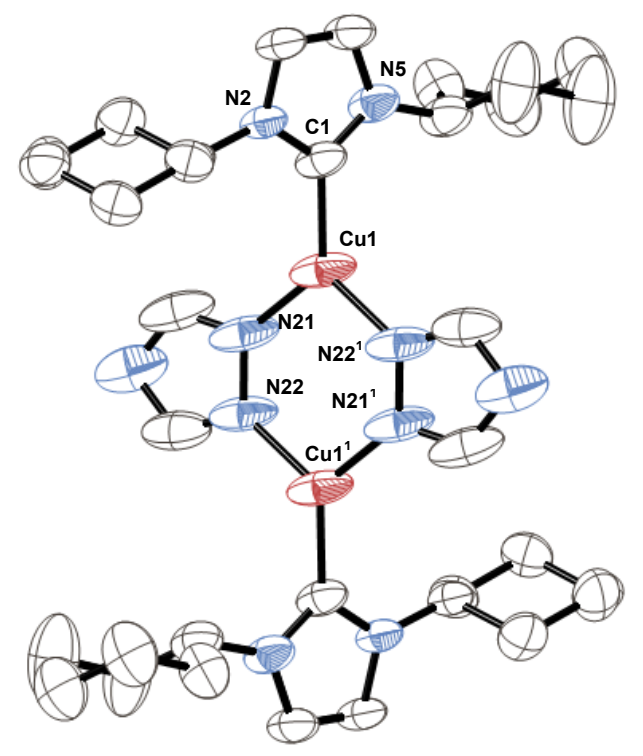

Figure S5. Molecular structure of 2e. All solvent molecules and hydrogen atoms are omitted for clarity. Thermal ellipsoids are shown at the $50 \%$ probability level.

\begin{tabular}{|c|c|}
\hline Complex & $2 \mathrm{e}$ \\
\hline CCDC number & 1442426 \\
\hline Emperical formula & $\mathrm{C}_{35} \mathrm{H}_{54} \mathrm{Cl}_{2} \mathrm{Cu}_{2} \mathrm{~N}_{10}$ \\
\hline Formula Weight & 812.88 \\
\hline Crystal color, Habit & colorless, prism \\
\hline Temperature $(\mathrm{K})$ & 173.15 \\
\hline Crystal system & tetragonal \\
\hline Space group & $\mathrm{I}_{1} / \mathrm{a}(\# 88)$ \\
\hline Unit cell dim. & $0.270 \times 0.160 \times 0.120 \mathrm{~mm}$ \\
\hline Lattice type & I-centered \\
\hline $\begin{array}{l}\text { Lattice parameter } \\
\qquad \text { a,b,c }(\AA)\end{array}$ & $\begin{array}{l}a=20.4817(14) \\
c=21.6349(17)\end{array}$ \\
\hline Volume $(\AA)^{3}$ & $\mathrm{~V}=9075.8(11)$ \\
\hline $\mathrm{Z}$ & 8 \\
\hline Density calculated & $1.190 \mathrm{~g} / \mathrm{cm}^{3}$ \\
\hline Absorption coefficient $\left(\mathrm{cm}^{-1}\right)$ & $10.884 \mathrm{~cm}^{-1}$ \\
\hline $\mathrm{F}(000)$ & 3408.00 \\
\hline Diffractometer & XtaLAB P200 \\
\hline Radiation & $\begin{array}{l}\operatorname{MoK} \alpha(\lambda=0.71075 \AA) \\
\text { multi-layer mirror monochromated }\end{array}$ \\
\hline Voltage, Current & $45 \mathrm{kV}, 66 \mathrm{~mA}$ \\
\hline Theta range for data collection $\left(^{\circ}\right)$ & $2 \theta_{\max }=50.7^{\circ}$ \\
\hline Reflexions collected & $\begin{array}{c}\text { Total: } 55142 \\
\text { Unique: } 4137\left(\mathrm{R}_{\mathrm{int}}=0.0299\right)\end{array}$ \\
\hline
\end{tabular}




\begin{tabular}{|c|c|}
\hline \multirow{2}{*}{ Correction } & Lorentz-polarization \\
& Absorption \\
& (trans. factors: $0.785-0.878)$ \\
\hline Structure solution & Direct Methods (SIR2004) \\
\hline Refinement method & Full-matrix least-squares on $\mathrm{F}^{2}$ \\
\hline Anomalous dispersion & All non-hydrogen atoms \\
\hline No. Observations (all reflections) & 4137 \\
\hline No. variables & 253 \\
\hline Reflection/parameter ratio & 16.35 \\
\hline Goodness-of-fit on $\mathrm{F}^{2}$ & 1.071 \\
\hline Final R indices [I>2sigma(I)] & 0.0606 \\
\hline R indices (all data) & 0.0720 \\
\hline Maximum peak in Final Diff Map $\left(\mathrm{e} . \AA^{-3}\right)$ & $0.72 \mathrm{e}^{-} / \AA^{3}$ \\
\hline Minimum peak in Final Diff Map $\left(\mathrm{e} . \AA^{-3}\right)$ & $-1.02 \mathrm{e}^{-} / \AA^{3}$ \\
\hline Max shift/error in final cycle & 0.000 \\
\hline
\end{tabular}

Table S7. Selected bond distances, angles and torsion angles for $\mathbf{2 e}$

\begin{tabular}{|c|c|c|}
\hline \multicolumn{3}{|c|}{ Bond distances $(\AA)$} \\
\hline $\mathrm{Cu} 1-\mathrm{C} 1=1.911(4)$ & $\mathrm{Cu} 1-\mathrm{N} 22^{1}=1.981(4)$ & $\mathrm{Cu} 1-\mathrm{N} 21=1.987(4)$ \\
\hline \multicolumn{3}{|c|}{ Bond angles $\left(^{\circ}\right)$} \\
\hline $\mathrm{N} 22^{1}-\mathrm{Cu} 1-\mathrm{C} 1=126.45(18)$ & $\mathrm{N} 21-\mathrm{Cu} 1-\mathrm{C} 1=127.02(17)$ & $\mathrm{N} 21-\mathrm{Cu} 1-\mathrm{N} 22^{1}=106.32(17)$ \\
\hline \multicolumn{3}{|c|}{ Torsional angles $\left(^{\circ}\right)$} \\
\hline $\mathrm{N} 21-\mathrm{Cu} 1-\mathrm{C} 1-\mathrm{N} 2=59.9(4)$ & $\mathrm{N} 22^{1}-\mathrm{Cu} 1-\mathrm{C} 1-\mathrm{N} 2=-114.0(3)$ & $\mathrm{N} 21-\mathrm{Cu} 1-\mathrm{C} 1-\mathrm{N} 5=-126.2(3)$ \\
\hline $\mathrm{N} 22^{1}-\mathrm{Cu} 1-\mathrm{C} 1-\mathrm{N} 5=59.8(4)$ & $\mathrm{Cu} 1-\mathrm{N} 21-\mathrm{N} 22-\mathrm{Cu} 1^{1}=0.7(6)$ & $\mathrm{N} 21-\mathrm{Cu} 1-\mathrm{N} 22^{1}-\mathrm{N} 21^{1} 0.5(4)$ \\
\hline $\mathrm{N} 22^{1}-\mathrm{Cu} 1-\mathrm{N} 21-\mathrm{N} 22=-0.5(3)$ & $\mathrm{C} 1-\mathrm{Cu} 1-\mathrm{Cu} 1^{1}=\mathbf{1 7 6 . 1}(\mathbf{1 1})$ & \\
\hline
\end{tabular}




\section{Calculation of $\% V_{\text {Bur }}$}

In complexes $\mathbf{2 a}, \mathbf{2 c}$ and $\mathbf{2 e}$ the two NHC ligands showed the same value of $\% V_{\mathrm{Bur}}$. For complexes $\mathbf{2 b}$ and $\mathbf{2 d}$ the $\% V_{\mathrm{Bur}}$ values of the two NHC ligands are different and both values are reported below. In the latter case the label of the carbene carbon (referred to the corresponding crystal structure) of the NHC ligand considered was specified.

\section{$[\mathrm{Cu}(\operatorname{trz})(\operatorname{IPr})]_{2}, 2 \mathrm{a}$}

\begin{tabular}{|c|c|c|c|}
\hline V Free & V Buried & V Total & V Exact \\
\hline 110.9 & 68.6 & 179.5 & 179.6 \\
\hline
\end{tabular}

\begin{tabular}{|c|c|c|}
\hline \%V_Free & \%V_Bur & \% Tot/Ex \\
\hline 61.8 & $\mathbf{3 8 . 2}$ & 100.0 \\
\hline
\end{tabular}

\begin{tabular}{|c|c|c|c|c|c|}
\hline xy & V_f & V_b & V_t & \%V_f & \%V_b \\
\hline-- & 23.9 & 21.0 & 44.9 & 53.3 & $\mathbf{4 6 . 7 2}$ \\
\hline-+ & 32.1 & 12.8 & 44.9 & 71.5 & $\mathbf{2 8 . 5 2}$ \\
\hline++ & 28.2 & 16.7 & 44.9 & 62.8 & $\mathbf{3 7 . 2 2}$ \\
\hline+- & 26.7 & 18.1 & 44.9 & 59.6 & $\mathbf{4 0 . 2 2}$ \\
\hline
\end{tabular}

\begin{tabular}{|c|c|c|c|c|c|c|c|c|}
\hline xy & --- & -+- & ++- & +- & --+ & -++ & +++ & +-+ \\
\hline \%V_b & 5.9 & 0.0 & 1.1 & 0.6 & 85.9 & 55.9 & 71.9 & 78.6 \\
\hline
\end{tabular}

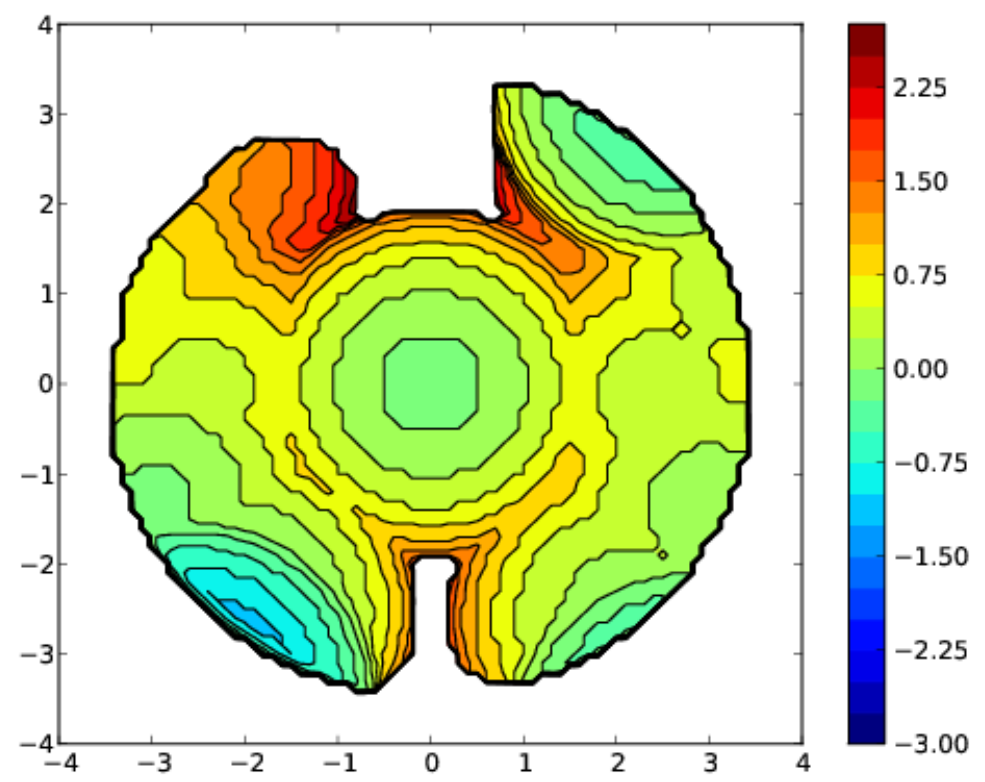

IMes ligand bearing $\mathrm{C} 1$ as carbon of the carbene in $[\mathrm{Cu}(\operatorname{trz})(\mathrm{IMes})]_{2}, 2 \mathrm{~b}$ 


\begin{tabular}{|c|c|c|c|}
\hline V Free & V Buried & V Total & V Exact \\
\hline 111.4 & 68.1 & 179.5 & 179.6 \\
\hline
\end{tabular}

\begin{tabular}{|c|c|c|}
\hline \%V_Free & \%V_Bur & \% Tot/Ex \\
\hline 62.1 & $\mathbf{3 7 . 9}$ & 100.0 \\
\hline
\end{tabular}

\begin{tabular}{|c|c|c|c|c|c|}
\hline xy & V_f & V_b & V_t & \% V_f & \% V_b \\
\hline-- & 30.7 & 14.2 & 44.9 & 68.3 & $\mathbf{3 1 . 6 6}$ \\
\hline-+ & 25.3 & 19.5 & 44.9 & 56.5 & $\mathbf{4 3 . 5 4}$ \\
\hline++ & 30.8 & 14.0 & 44.9 & 68.7 & $\mathbf{3 1 . 3 0}$ \\
\hline+- & 24.6 & 20.3 & 44.9 & 54.8 & $\mathbf{4 5 . 2 0}$ \\
\hline
\end{tabular}

\begin{tabular}{|c|c|c|c|c|c|c|c|c|}
\hline $\mathrm{xy}$ & --- & -+- & ++- & +-- & --+ & -++ & +++ & +-+ \\
\hline \%V_b & 0.0 & 1.1 & 0.0 & 2.9 & 62.0 & 83.9 & 61.3 & 85.8 \\
\hline
\end{tabular}

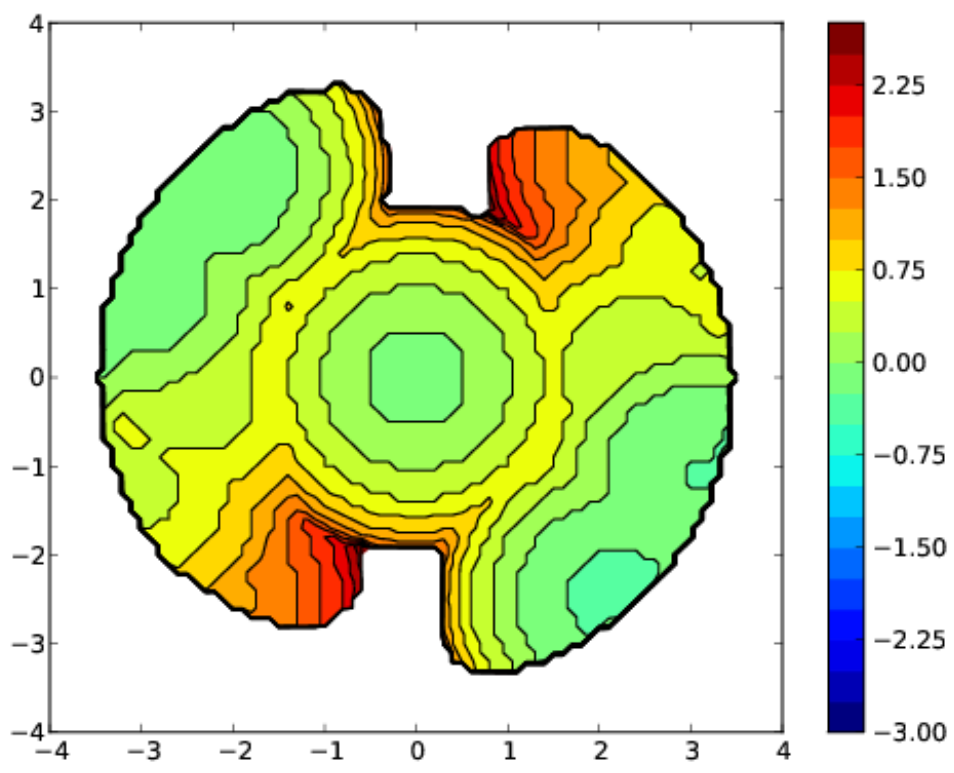




\begin{tabular}{|c|c|c|c|}
\hline V Free & V Buried & V Total & V Exact \\
\hline 113.8 & 65.7 & 179.5 & 179.6 \\
\hline
\end{tabular}

\begin{tabular}{|c|c|c|}
\hline \%V_Free & \%V_Bur & \% Tot/Ex \\
\hline 63.4 & $\mathbf{3 6 . 6}$ & 100.0 \\
\hline
\end{tabular}

\begin{tabular}{|c|c|c|c|c|c|}
\hline xy & V_f & V_b & V_t & \% V_f & \%V_b \\
\hline-- & 26.4 & 18.4 & 44.9 & 58.9 & $\mathbf{4 1 . 1 0}$ \\
\hline-+ & 28.7 & 16.1 & 44.9 & 64.1 & $\mathbf{3 5 . 9 3}$ \\
\hline++ & 27.7 & 17.1 & 44.9 & 61.8 & $\mathbf{3 8 . 1 6}$ \\
\hline+- & & 30.9 & 14.0 & 44.9 & 68.9 \\
\hline
\end{tabular}

\begin{tabular}{|c|c|c|c|c|c|c|c|c|}
\hline $\mathrm{xy}$ & --- & -+- & ++- & +-- & --+ & -++ & +++ & +-+ \\
\hline \%V_b & 0.0 & 0.0 & 0.0 & 0.0 & 80.5 & 70.4 & 74.7 & 61.0 \\
\hline
\end{tabular}

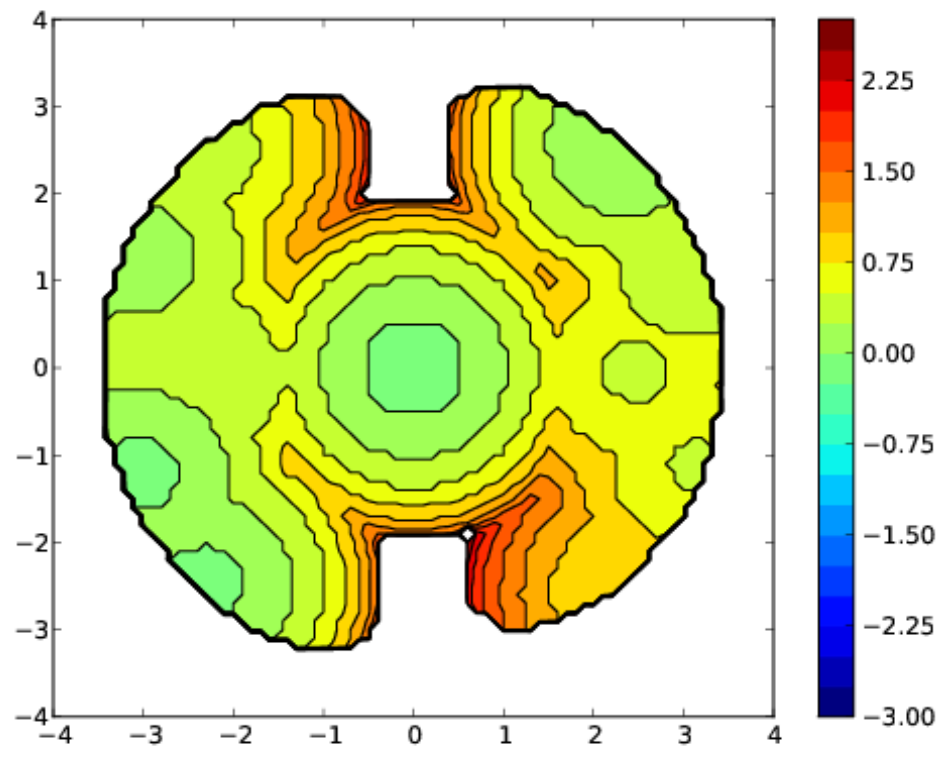




\section{$\left[\mathrm{Cu}(\text { trz)(SIMes) }]_{2}, 2 \mathrm{c}\right.$}

\begin{tabular}{|c|c|c|c|}
\hline V Free & V Buried & V Total & Exact \\
\hline 111.1 & 68.4 & 179.5 & 179.6 \\
\hline
\end{tabular}

\begin{tabular}{|c|c|c|}
\hline \%V_Free & \%V_Bur & \% Tot/Ex \\
\hline 61.9 & $\mathbf{3 8 . 1}$ & 100.0 \\
\hline
\end{tabular}

\begin{tabular}{|c|c|c|c|c|c|}
\hline xy & V_f & V_b & V_t & \%V_f & \% V_b \\
\hline-- & 23.5 & 21.4 & 44.9 & 52.4 & $\mathbf{4 7 . 5 9}$ \\
\hline-+ & 31.5 & 13.4 & 44.9 & 70.2 & $\mathbf{2 9 . 8 1}$ \\
\hline++ & 23.2 & 21.6 & 44.9 & 51.8 & $\mathbf{4 8 . 1 7}$ \\
\hline+- & 32.8 & 12.0 & 44.9 & 73.2 & $\mathbf{2 6 . 8 0}$ \\
\hline
\end{tabular}

\begin{tabular}{|c|c|c|c|c|c|c|c|c|}
\hline $\mathrm{xy}$ & --- & -+- & ++- & +- & --+ & -++ & +++ & +-+ \\
\hline \%V_b & 4.5 & 0.0 & 2.5 & 0.0 & 88.9 & 58.4 & 91.9 & 52.5 \\
\hline
\end{tabular}

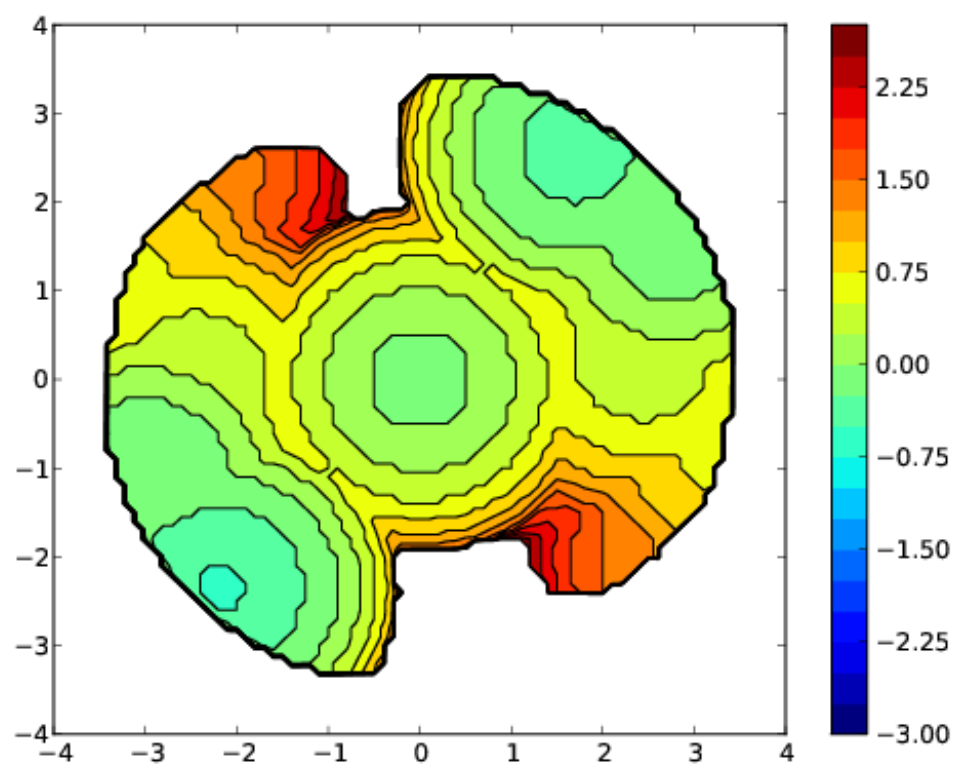


$I^{t} B u$ ligand bearing $\mathrm{C} 1$ as carbon of the carbene in $\left[\mathrm{Cu}(\operatorname{trz})\left(I^{t} B u\right)\right]_{2}, 2 d$

\begin{tabular}{|c|c|c|c|}
\hline V Free & V Buried & V Total & V Exact \\
\hline 107.6 & 71.9 & 179.5 & 179.6 \\
\hline
\end{tabular}

\begin{tabular}{|c|c|c|}
\hline \%V_Free & \%V_Bur & \% Tot/Ex \\
\hline 59.9 & $\mathbf{4 0 . 1}$ & 100.0 \\
\hline
\end{tabular}

\begin{tabular}{|c|c|c|c|c|c|}
\hline xy & V_f & V_b & V_t & \%V_f & \% V_b \\
\hline-- & 26.1 & 18.8 & 44.9 & 58.2 & $\mathbf{4 1 . 8 5}$ \\
\hline-+ & 28.4 & 16.4 & 44.9 & 63.4 & $\mathbf{3 6 . 6 2}$ \\
\hline++ & 26.2 & 18.7 & 44.9 & 58.4 & $\mathbf{4 1 . 6 3}$ \\
\hline+- & 26.9 & 18.0 & 44.9 & 59.9 & $\mathbf{4 0 . 1 4}$ \\
\hline
\end{tabular}

\begin{tabular}{|c|c|c|c|c|c|c|c|c|}
\hline xy & --- & -+- & ++- & +-- & --+ & -++ & +++ & +-+ \\
\hline \%V_b & 12.8 & 7.3 & 6.3 & 2.5 & 69.7 & 64.7 & 75.4 & 76.3 \\
\hline
\end{tabular}

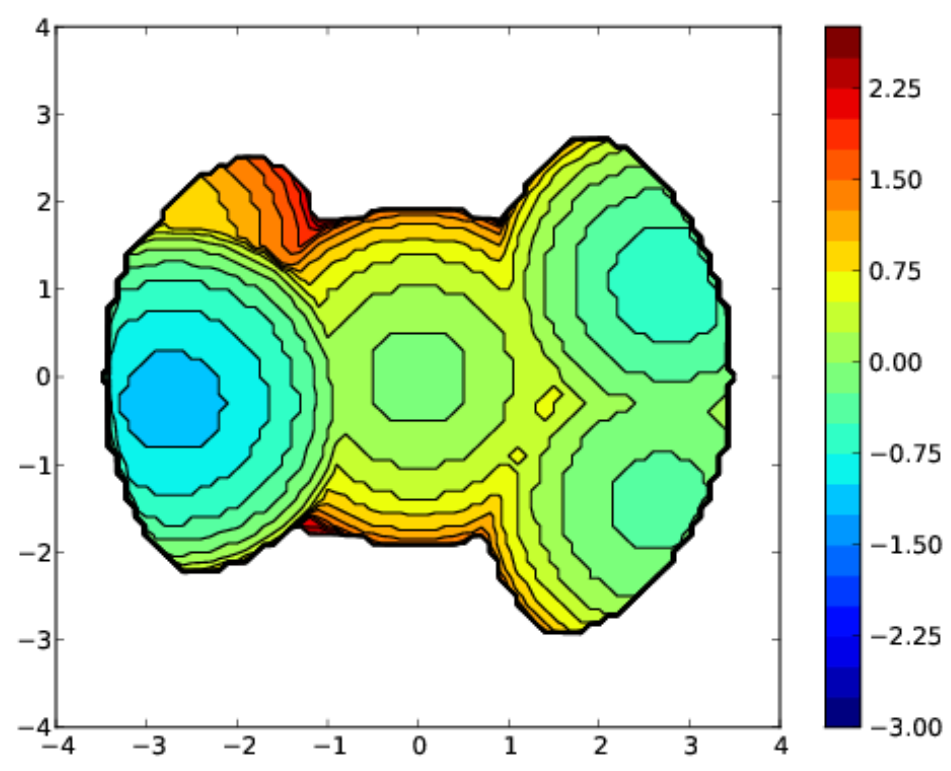


$I^{t} B u$ ligand bearing $C 41$ as carbon of the carbene in $\left[\mathrm{Cu}(\operatorname{trz})\left(\mathrm{I}^{\mathrm{t} B u}\right)\right]_{2}, 2 \mathrm{~d}$

\begin{tabular}{|c|c|c|c|}
\hline V Free & V Buried & V Total & V Exact \\
\hline 109.1 & 70.4 & 179.5 & 179.6 \\
\hline
\end{tabular}

\begin{tabular}{|c|c|c|}
\hline \%V_Free & \%V_Bur & \% Tot/Ex \\
\hline 60.8 & $\mathbf{3 9 . 2}$ & 100.0 \\
\hline
\end{tabular}

\begin{tabular}{|c|c|c|c|c|c|}
\hline xy & V_f & V_b & V_t & \%V_f & \%V_b \\
\hline-- & 25.9 & 19.0 & 44.9 & 57.6 & $\mathbf{4 2 . 3 7}$ \\
\hline-+ & 28.3 & 16.6 & 44.9 & 63.1 & $\mathbf{3 6 . 9 0}$ \\
\hline++ & 27.4 & 17.4 & 44.9 & 61.2 & $\mathbf{3 8 . 8 2}$ \\
\hline+- & 27.5 & 17.4 & 44.9 & 61.2 & $\mathbf{3 8 . 7 9}$ \\
\hline
\end{tabular}

\begin{tabular}{|c|c|c|c|c|c|c|c|c|}
\hline xy & --- & -+- & ++- & +-- & --+ & -++ & +++ & +-+ \\
\hline$\% \mathrm{~V} \_b$ & 12.3 & 4.9 & 9.7 & 10.5 & 71.2 & 67.5 & 66.7 & 65.9 \\
\hline
\end{tabular}

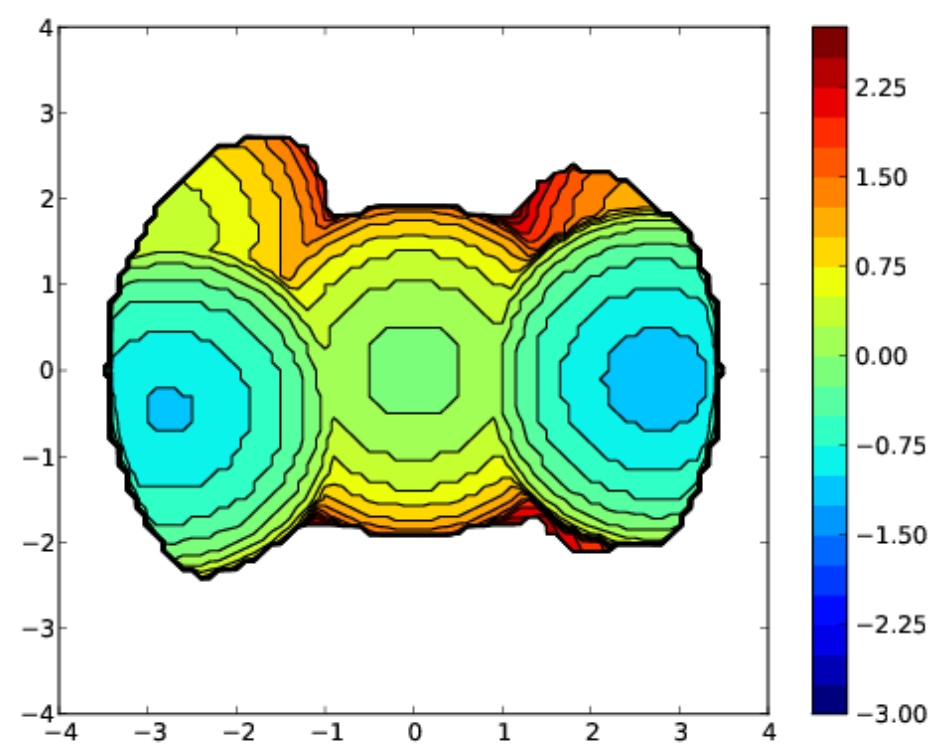




\section{$[\mathrm{Cu}(\operatorname{trz})(\mathrm{ICy})]_{2}, 2 \mathrm{e}$}

\begin{tabular}{|c|c|c|c|}
\hline V Free & V Buried & V Total & V Exact \\
\hline 128.3 & 51.2 & 179.5 & 179.6 \\
\hline
\end{tabular}

\begin{tabular}{|c|c|c|}
\hline \%V_Free & \%V_Bur & \% Tot/Ex \\
\hline 71.5 & $\mathbf{2 8 . 5}$ & 100.0 \\
\hline
\end{tabular}

\begin{tabular}{|c|c|c|c|c|c|}
\hline xy & V_f & V_b & V_t & \%V_f & \% V_b \\
\hline-- & 31.2 & 13.7 & 44.9 & 69.5 & $\mathbf{3 0 . 5 5}$ \\
\hline-+ & 32.8 & 12.1 & 44.9 & 73.1 & $\mathbf{2 6 . 9 3}$ \\
\hline++ & 31.9 & 13.0 & 44.9 & 71.1 & $\mathbf{2 8 . 8 9}$ \\
\hline+- & 32.4 & 12.4 & 44.9 & 72.3 & $\mathbf{2 7 . 6 8}$ \\
\hline
\end{tabular}

\begin{tabular}{|c|c|c|c|c|c|c|c|c|}
\hline $\mathrm{xy}$ & --- & -+- & ++- & +- & --+ & -++ & +++ & +-+ \\
\hline$\% \mathrm{~V} \_\mathrm{b}$ & 0.0 & 0.0 & 0.0 & 0.0 & 59.8 & 52.7 & 56.6 & 54.2 \\
\hline
\end{tabular}

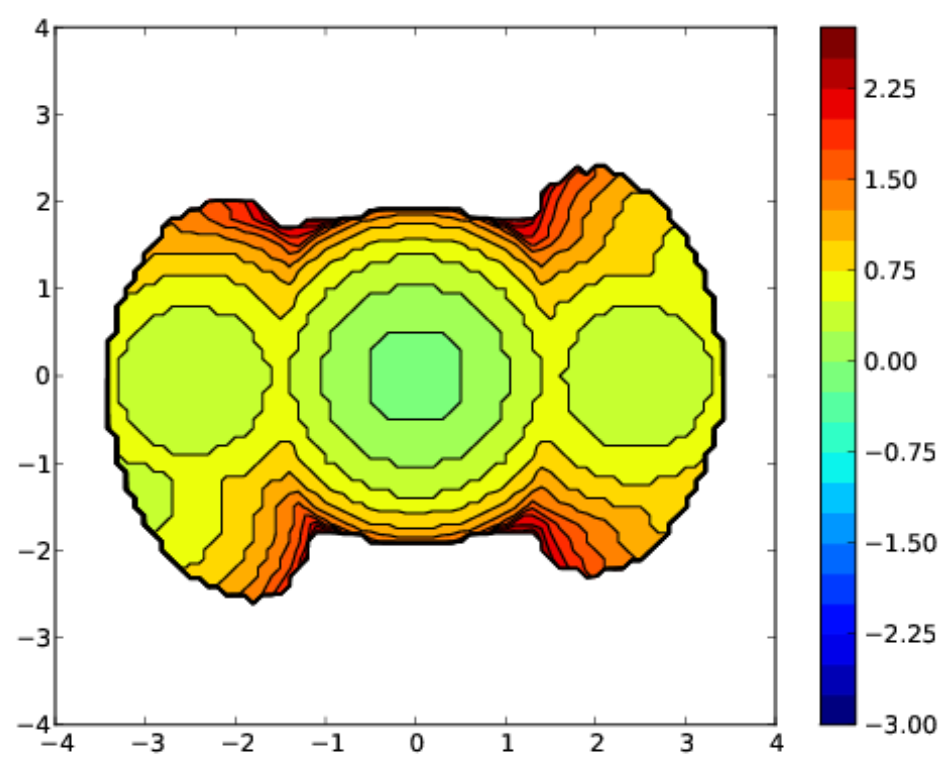




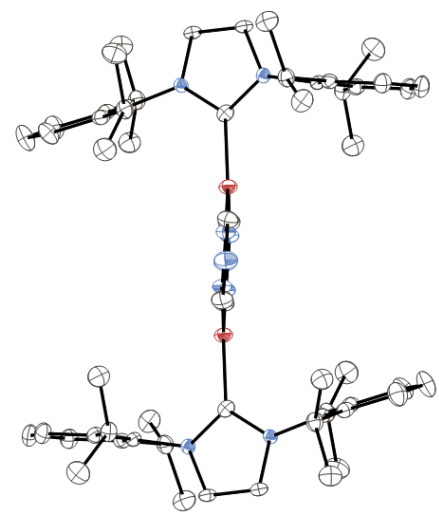

2a

$\% V_{\text {Bur }}$

Torsional angle $\left(^{\circ}\right)$

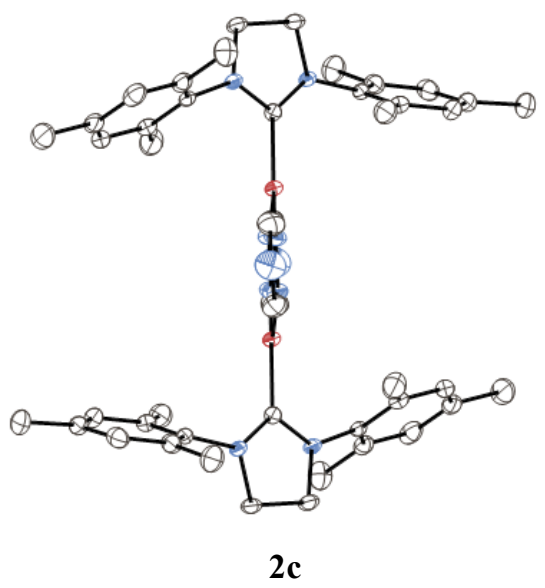

$\% V_{\text {Bur }}$

Torsional angle $\left(^{\circ}\right)$

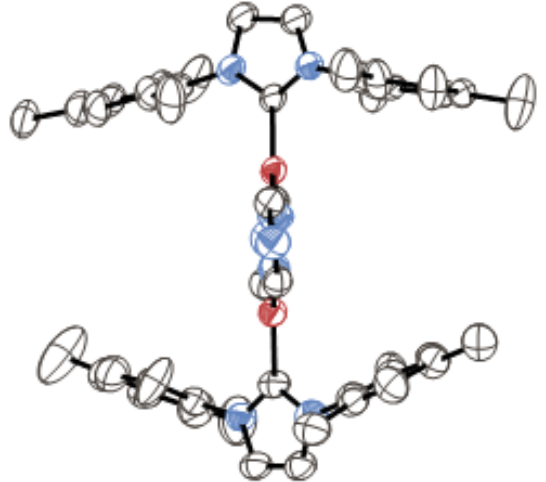

2b

37.9 and 36.6

$178.0(7)$

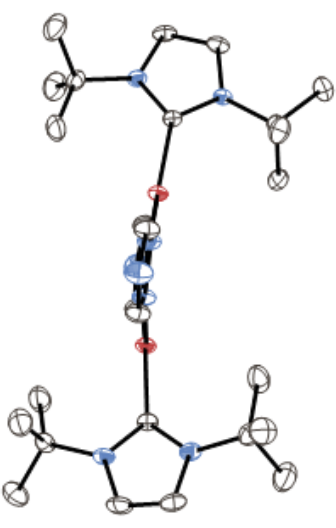

2d

40.1 and 39.2

$161.76(4)$

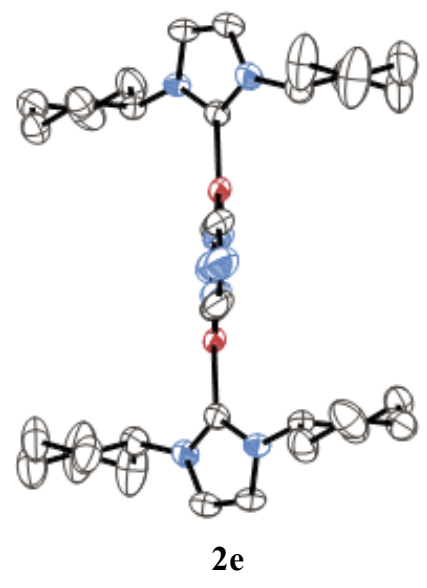

$\% V_{\text {Bur }}$

28.5

Torsional angle $\left(^{\circ}\right)$

$176.1(11)$

Figure S6. Percent buried volumes and torsion angles of $\mathbf{2 a - 2 e}$ 


\section{Hydrosilylation of ketones catalysed by dimeric copper complexes}

In a glovebox, a screw capped vial was charged with the catalyst, the solvent, dicyclohexylketone 3a and the silane. The reaction mixture was stirred at the indicated temperature and time. The conversion was monitored by GC analysis of reaction aliquots.

Table S8. Screening of catalysts with different silanes ${ }^{[a]}$<smiles>O=C(C1CCCCC1)C1CCCCC1</smiles>
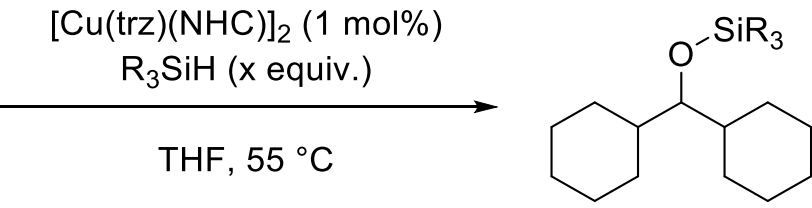

\begin{tabular}{|c|c|c|c|c|c|}
\hline Entry & Catalyst & Silane & Amount silane (equiv.) & Time (h) & Conversion $(\%)^{[\mathrm{b}]}$ \\
\hline 1 & - & $\mathrm{Me}(\mathrm{OEt})_{2} \mathrm{SiH}$ & 2 & 0.75 & 0 \\
\hline 2 & {$[\mathrm{Cu}(\operatorname{trz})(\mathrm{IPr})]_{2}$} & $\mathrm{Me}(\mathrm{OEt})_{2} \mathrm{SiH}$ & 2 & 0.75 & $>99$ \\
\hline 3 & {$[\mathrm{Cu}(\operatorname{trz})(\mathrm{IMes})]_{2}$} & $\mathrm{Me}(\mathrm{OEt})_{2} \mathrm{SiH}$ & 2 & 0.75 & 95 \\
\hline 4 & {$[\mathrm{Cu}(\operatorname{trz})(\mathrm{SIMes})]_{2}$} & $\mathrm{Me}(\mathrm{OEt})_{2} \mathrm{SiH}$ & 2 & 0.75 & 84 \\
\hline 5 & {$\left[\mathrm{Cu}(\operatorname{trz})\left(\mathrm{I}^{\mathrm{t}} \mathrm{Bu}\right)\right]_{2}$} & $\mathrm{Me}(\mathrm{OEt})_{2} \mathrm{SiH}$ & 2 & 0.75 & 0 \\
\hline 6 & {$[\mathrm{Cu}(\operatorname{trz})(\mathrm{ICy})]_{2}$} & $\mathrm{Me}(\mathrm{OEt})_{2} \mathrm{SiH}$ & 2 & 0.75 & 5 \\
\hline 7 & {$[\mathrm{Cu}(\operatorname{trz})(\mathrm{IPr})]_{2}$} & $\mathrm{Me}(\mathrm{OEt})_{2} \mathrm{SiH}$ & 1.5 & 0.75 & $>99$ \\
\hline
\end{tabular}

${ }^{[\mathrm{a}]}$ Reaction conditions: dicyclohexylketone $\mathbf{3 a}(0.5 \mathrm{mmol}), \mathrm{Me}(\mathrm{OEt})_{2} \mathrm{SiH}(0.75-1 \mathrm{mmol}),[\mathrm{Cu}(\mathrm{trz})(\mathrm{NHC})]_{2}(1 \mathrm{~mol} \%)$, THF $(0.75 \mathrm{~mL}), 55^{\circ} \mathrm{C} .{ }^{[b]}$ Determined by GC, based on dicyclohexylketone, average of 2 reactions. 
Screening of catalyst loading was performed using freshly prepared stock solutions of the catalyst dissolved in the same solvent used for the reaction. All reactions were performed twice using two different freshly prepared stock solutions.

Preparation of stock solution (A) for $0.5 \mathrm{~mol} \%$ and $0.25 \mathrm{~mol} \%$ tests.

$5.26 \mathrm{mg}$ of $[\mathrm{Cu}(\mathrm{trz})(\mathrm{IPr})]_{2}$ were dissolved in $2 \mathrm{~mL}$ and $500 \mu \mathrm{L}$ were taken for $0.5 \mathrm{~mol} \%$ reaction or $250 \mu \mathrm{L}$ for 0.25 mol\% reaction.

Preparation stock solution (B) for $0.1 \mathrm{~mol} \%, 0.05 \mathrm{~mol} \%$ and $0.01 \mathrm{~mol} \%$ tests.

$5.26 \mathrm{mg}$ of $[\mathrm{Cu}(\mathrm{trz})(\mathrm{IPr})]_{2}$ were dissolved in $4 \mathrm{~mL}$ of solvent, $200 \mu \mathrm{L}$ were taken for $0.1 \mathrm{~mol} \%$ reaction; $100 \mu \mathrm{L}$ for 0.05 $\mathrm{mol} \%$ reaction or $20 \mu \mathrm{L}$ for $0.01 \mathrm{~mol} \%$ reaction.

Table S9. Optimisation of catalyst loading ${ }^{[\mathrm{a}]}$<smiles>O=C(C1CCCCC1)C1CCCCC1</smiles>
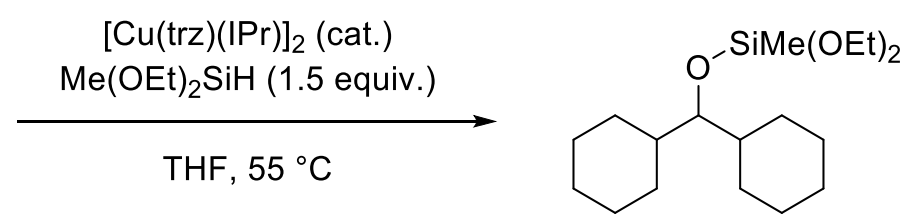

\begin{tabular}{llll} 
Entry & Catalyst loading $(\mathrm{mol} \%)$ & Time $(\mathrm{h})$ & GC conversion $(\%)^{[\mathrm{b}]}$ \\
\hline 1 & 0.5 & 0.75 & $>99$ \\
2 & 0.25 & 0.75 & $>99$ \\
3 & 0.1 & 0.75 & $>99$ \\
4 & 0.05 & 0.75 & 85 \\
5 & 0.05 & 2 & $>99$ \\
6 & 0.01 & 0.75 & 0 \\
7 & 0.01 & 2 & 0
\end{tabular}

${ }^{[\mathrm{a}]}$ Reaction conditions: 3a $(0.5 \mathrm{mmol}), \mathrm{Me}(\mathrm{OEt})_{2} \mathrm{SiH}(0.75 \mathrm{mmol}),[\mathrm{Cu}(\mathrm{trz})(\mathrm{IPr})]_{2}(0.01-0.5 \mathrm{~mol} \%), \mathrm{THF}(0.75 \mathrm{~mL}), 55$ ${ }^{\circ} \mathrm{C}, 0.75-2$ h. ${ }^{[\mathrm{b}]}$ Determined by GC, based on dicyclohexylketone, average of 2 reactions.

Table S10. Optimisation of amount of silane ${ }^{[a]}$<smiles>O=C(C1CCCCC1)C1CCCCC1</smiles>
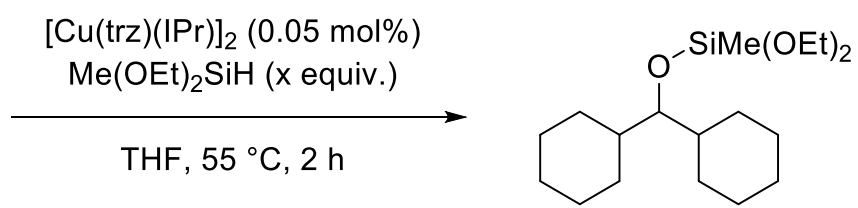

\begin{tabular}{lll} 
Entry & Silane (equiv.) & GC conversion $(\%)^{[\mathrm{b}]}$ \\
\hline 1 & 1.3 & $>99$ \\
2 & 1.1 & $>99$ \\
\hline${ }^{[\mathrm{a}]}$ Reaction conditions: 3a $(0.5 \mathrm{mmol}), \mathrm{Me}(\mathrm{OEt})_{2} \mathrm{SiH}(0.55-0.65 \mathrm{mmol}),[\mathrm{Cu}(\mathrm{trz})(\mathrm{IPr})]_{2}(0.05 \mathrm{~mol} \%), \mathrm{THF}(0.75 \mathrm{~mL})$, \\
$55^{\circ} \mathrm{C}, 2$ h. ${ }^{[\mathrm{b}]}$ Determined by GC, based on dicyclohexylketone, average of 2 reactions.
\end{tabular}

Table S11. Optimisation of solvent and temperature ${ }^{[\mathrm{a}]}$ 


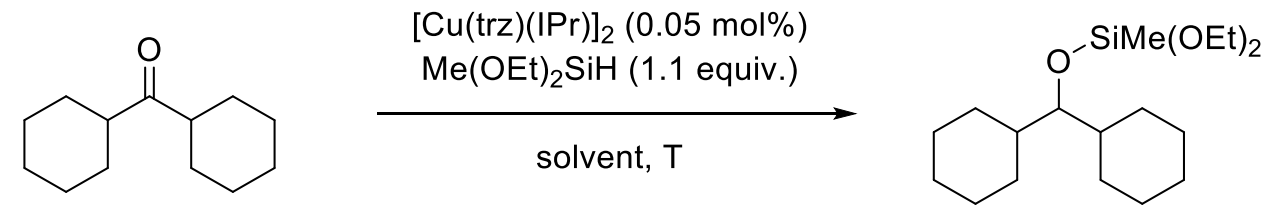

\begin{tabular}{llllll} 
Entry & Solvent & Silane & Time $(\mathrm{h})$ & $\mathrm{GC}$ conversion $(\%)^{[\mathrm{b}]}$ & $\mathrm{T}\left({ }^{\circ} \mathrm{C}\right)$ \\
\hline 1 & THF & $\mathrm{Me}(\mathrm{OEt})_{2} \mathrm{SiH}$ & 2 & $>99$ & 55 \\
2 & 1,4 -Dioxane & $\mathrm{Me}(\mathrm{OEt})_{2} \mathrm{SiH}$ & 2 & 93 & 55 \\
3 & Methyl $t$-butyl ether & $\mathrm{Me}(\mathrm{OEt})_{2} \mathrm{SiH}$ & 2 & 91 & 55 \\
4 & Toluene & $\mathrm{Me}(\mathrm{OEt})_{2} \mathrm{SiH}$ & 2 & 82 & 55 \\
5 & DME & $\mathrm{Me}(\mathrm{OEt})_{2} \mathrm{SiH}$ & 2 & 73 & 55 \\
6 & $\mathrm{MCE}$ & $\mathrm{Me}(\mathrm{OEt})_{2} \mathrm{SiH}$ & 2 & 0 & 55 \\
7 & $\mathrm{MeOH}$ & $\mathrm{Me}(\mathrm{OEt})_{2} \mathrm{SiH}$ & 2 & 0 & 55 \\
8 & $\mathrm{IPrOH}$ & $\mathrm{Me}(\mathrm{OEt})_{2} \mathrm{SiH}$ & 2 & 0 & 55 \\
9 & $t$ BuOH & $\mathrm{Me}(\mathrm{OEt})_{2} \mathrm{SiH}$ & 2 & 0 & 55 \\
\hline 10 & THF & $\mathrm{Me}(\mathrm{OEt})_{2} \mathrm{SiH}$ & 3 & 92 & 45 \\
11 & $1,4-$ Dioxane & $\mathrm{Me}(\mathrm{OEt})_{2} \mathrm{SiH}$ & 3 & 87 & 45 \\
12 & Methyl $t$-butyl ether & $\mathrm{Me}(\mathrm{OEt})_{2} \mathrm{SiH}$ & 3 & 81 & 45 \\
13 & Toluene & $\mathrm{Me}(\mathrm{OEt})_{2} \mathrm{SiH}$ & 3 & 51 & 45 \\
\hline 14 & THF & $\mathrm{Me}(\mathrm{OEt})_{2} \mathrm{SiH}$ & 4 & 72 & 35 \\
15 & $1,4-$-Dioxane & $\mathrm{Me}(\mathrm{OEt})_{2} \mathrm{SiH}$ & 4 & 60 & 35 \\
16 & Methyl $t$-butyl ether & $\mathrm{Me}(\mathrm{OEt})_{2} \mathrm{SiH}$ & 4 & 74 & 35 \\
17 & Toluene & $\mathrm{Me}(\mathrm{OEt})_{2} \mathrm{SiH}$ & 4 & 13 & 35 \\
\hline
\end{tabular}

${ }^{[\mathrm{a}]}$ Reaction conditions: 3a $(0.5 \mathrm{mmol}), \mathrm{Me}(\mathrm{OEt}){ }_{2} \mathrm{SiH}(0.55 \mathrm{mmol}),[\mathrm{Cu}(\mathrm{trz})(\mathrm{IPr})]_{2}(0.05 \mathrm{~mol} \%)$, solvent $(0.75 \mathrm{~mL}), 2-4$ h. ${ }^{[\mathrm{b}]}$ Determined by GC, based on dicyclohexylketone, average of 2 reactions.

\section{Characterisation of the synthesised alcohols 5a-5s}

General procedure for the hydrosilylation of ketones using PMHS: In a glovebox, a screw capped vial was charged with $200 \mu \mathrm{L}$ of a stock solution of $[\mathrm{Cu} \text { (trz) IPr) }]_{2}(0.05 \mathrm{~mol} \%)$ in THF $(5.3 \mathrm{mg}$ in $4 \mathrm{~mL})$, THF (1.3 mL), ketone (1 mmol) and PMHS (2 mmol). The reaction mixture was stirred at $55{ }^{\circ} \mathrm{C}$ for the specified time. $\mathrm{MeOH}(1 \mathrm{~mL})$ and an aqueous solution of $\mathrm{NaOH}(2 \mathrm{M}, 2 \mathrm{~mL})$ were added and the reaction mixture was stirred at $25^{\circ} \mathrm{C}$ for 90 min. The crude product was washed with brine $(2 \times 10 \mathrm{~mL})$ and the aqueous layer was extracted with diethyl ether $(3 \mathrm{x} 10 \mathrm{~mL})$. The combined organic layers were dried over $\mathrm{MgSO}_{4}$, filtered and dried under vacuum to yield the desired product.

Dicyclohexylmethanol, $5 \mathbf{a}^{[1]}$<smiles>OC(C1CCCCC1)C1CCCCC1</smiles>

The general procedure, using $0.25 \mathrm{~mol} \%$ of $\mathbf{2 a}$, yielded the title compound as a colourless solid (190 $\mathrm{mg}, 97 \%)$.

${ }^{1}$ H NMR (500 MHz, CDCl 3 , 298K, TMS): $\delta(\mathrm{ppm})=3.05\left(\mathrm{t},{ }^{3} J_{\mathrm{H}-\mathrm{H}}=5.6 \mathrm{~Hz}, 1 \mathrm{H}, \mathrm{CH}\right), 1.87-0.94$ (m, 23H cyclohexyl and $\mathrm{OH})$.

${ }^{13} \mathbf{C}-\left\{{ }^{1} \mathbf{H}\right\}$ NMR (126 MHz, $\left.\mathbf{C D C l}_{3}, \mathbf{2 9 8 K}\right): \delta(\mathrm{ppm})=80.8(\mathrm{~s}, \mathrm{CH}), 40.2(\mathrm{~s}, \mathrm{CH}), 30.3\left(\mathrm{~s}, \mathrm{CH}_{2}\right), 27.7\left(\mathrm{~s}, \mathrm{CH}_{2}\right), 26.9(\mathrm{~s}$, $\left.\mathrm{CH}_{2}\right), 26.8\left(\mathrm{~s}, \mathrm{CH}_{2}\right), 26.5\left(\mathrm{~s}, \mathrm{CH}_{2}\right)$. 
<smiles>CC(O)c1ccccc1</smiles>

The general procedure yielded the title compound as a colourless oil (122 mg, 99\%).

${ }^{1}$ H NMR (500 MHz, CDCl 3 , 298K, TMS): $\delta(\mathrm{ppm})=7.39-7.34\left(\mathrm{~m}, 4 \mathrm{H}, \mathrm{C}_{\mathrm{Ar}} \mathrm{H}\right), 7.29-7.26\left(\mathrm{~m}, 1 \mathrm{H}, \mathrm{C}_{\mathrm{Ar}} \mathrm{H}\right), 4.09\left(\mathrm{q},{ }^{3} J_{\mathrm{H}-\mathrm{H}}\right.$ $=6.6 \mathrm{~Hz}, 1 \mathrm{H}, \mathrm{CH}), 1.85($ br s, $1 \mathrm{H}, \mathrm{OH}), 1.50\left(\mathrm{~d},{ }^{3} \mathrm{~J}_{\mathrm{HH}}=6.6 \mathrm{~Hz}, 3 \mathrm{H}, \mathrm{CH}_{3}\right)$.

${ }^{13} \mathbf{C}-\left\{{ }^{1} \mathbf{H}\right\}$ NMR (126 MHz, $\left.\mathbf{C D C l}_{3}, \mathbf{2 9 8 K}\right): \delta(\mathrm{ppm})=146.1\left(\mathrm{~s}, \mathrm{C}^{\mathrm{IV}}\right), 128.9\left(\mathrm{~s}, \mathrm{C}_{\mathrm{Ar}} \mathrm{H}\right), 127.8\left(\mathrm{~s}, \mathrm{C}_{\mathrm{Ar}} \mathrm{H}\right), 125.7\left(\mathrm{~s}, \mathrm{C}_{\mathrm{Ar}} \mathrm{H}\right)$, $70.8(\mathrm{~s}, \mathrm{CH}), 25.5\left(\mathrm{~s}, \mathrm{CH}_{3}\right)$.

\section{1-(4-Nitrophenyl)ethan-1-ol, $5 c^{[2]}$}<smiles>CC(O)c1ccc([N+](=O)[O-])cc1</smiles>

The general procedure yielded the title compound as a yellow oil (166 mg, 99\%).

${ }^{1}$ H NMR (400 MHz, CDCl 3 , 298K, TMS): $\delta(\mathrm{ppm})=8.23-8.19\left(\mathrm{~m}, 2 \mathrm{H}, \mathrm{C}_{\mathrm{Ar}} \mathrm{H}\right), 7.57-7.53\left(\mathrm{~m}, 2 \mathrm{H}, \mathrm{C}_{\mathrm{Ar}} \mathrm{H}\right), 5.03\left(\mathrm{q},{ }^{3} J_{\mathrm{H}-\mathrm{H}}\right.$ $=6.6 \mathrm{~Hz}, 1 \mathrm{H}, \mathrm{CH}), 2.05($ br s, $1 \mathrm{H}, \mathrm{OH}), 1.52\left(\mathrm{~d},{ }^{3} \mathrm{~J}_{\mathrm{HH}}=6.6 \mathrm{~Hz}, 3 \mathrm{H}, \mathrm{CH}_{3}\right)$.

${ }^{13} \mathbf{C}-\left\{{ }^{1} \mathbf{H}\right\}$ NMR (100 MHz, $\left.\mathbf{C D C l}_{3}, \mathbf{2 9 8 K}\right): \delta(\mathrm{ppm})=153.4\left(\mathrm{~s}, \mathrm{C}^{\mathrm{IV}}\right), 147.5\left(\mathrm{~s}, \mathrm{C}^{\mathrm{IV}}\right), 126.5\left(\mathrm{~s}, \mathrm{C}_{\mathrm{Ar}} \mathrm{H}\right), 124.1\left(\mathrm{~s}, \mathrm{C}_{\mathrm{Ar}} \mathrm{H}\right)$, $69.9(\mathrm{~s}, \mathrm{CH}), 25.9\left(\mathrm{~s}, \mathrm{CH}_{3}\right)$.

4-(1-Hydroxyethyl)benzonitrile, $5 d^{[2]}$<smiles>CC(O)c1ccc(C#N)cc1</smiles>

The general procedure yielded the title compound as a colourless oil (146 mg, 99\%).

${ }^{1}$ H NMR (500 MHz, CDCl 3 , 298K, TMS): $\delta(\mathrm{ppm})=7.64\left(\mathrm{~d},{ }^{3} J_{\mathrm{H}-\mathrm{H}}=8.1 \mathrm{~Hz}, 2 \mathrm{H}, \mathrm{C}_{\mathrm{Ar}} \mathrm{H}\right), 7.49\left(\mathrm{~d},{ }^{3} J_{\mathrm{H}-\mathrm{H}}=8.1 \mathrm{~Hz}, 2 \mathrm{H}\right.$, $\left.\mathrm{C}_{\mathrm{Ar}} \mathrm{H}\right), 4.97\left(\mathrm{q},{ }^{3} J_{\mathrm{H}-\mathrm{H}}=6.5 \mathrm{~Hz}, 1 \mathrm{H}, \mathrm{CH}\right), 2.02(\mathrm{br} \mathrm{s}, 1 \mathrm{H}, \mathrm{OH}), 1.50\left(\mathrm{~d},{ }^{3} J_{\mathrm{H}-\mathrm{H}}=6.5 \mathrm{~Hz}, 3 \mathrm{H}, \mathrm{CH}_{3}\right)$.

${ }^{13} \mathrm{C}-\left\{{ }^{1} \mathrm{H}\right\}$ NMR (126 MHz, $\left.\mathbf{C D C l}_{3}, \mathbf{2 9 8 K}\right): \delta(\mathrm{ppm})=151.4\left(\mathrm{~s}, \mathrm{C}^{\mathrm{IV}}\right), 132.7\left(\mathrm{~s}, C_{\mathrm{Ar}} \mathrm{H}\right), 126.4\left(\mathrm{~s}, C_{\mathrm{Ar}} \mathrm{H}\right), 119.2\left(\mathrm{~s}, \mathrm{C}^{\mathrm{IV}}\right)$, $111.4\left(\mathrm{~s}, \mathrm{C}^{\mathrm{IV}}\right), 70.0(\mathrm{~s}, \mathrm{CH}), 25.8\left(\mathrm{~s}, \mathrm{CH}_{3}\right)$.

1-(4-Chlorophenyl)ethan-1-ol, $5 \mathrm{e}^{[3]}$<smiles>CC(O)c1ccc(Cl)cc1</smiles>

The general procedure yielded the title compound as a colourless oil (144 mg, 92\%). 
${ }^{1} \mathrm{H}$ NMR (400 MHz, $\mathbf{C D C l}_{3}, \mathbf{2 9 8 K}$, TMS): $\delta(\mathrm{ppm})=7.31\left(\mathrm{~s}, 4 \mathrm{H}, \mathrm{C}_{\mathrm{Ar}} \mathrm{H}\right), 4.89\left(\mathrm{q},{ }^{3} J_{\mathrm{H}-\mathrm{H}}=6.5 \mathrm{~Hz}, 1 \mathrm{H}, \mathrm{CH}\right), 1.84(\mathrm{br} \mathrm{s}$, $1 \mathrm{H}, \mathrm{OH}), 1.48\left(\mathrm{~d},{ }^{3} J_{\mathrm{H}-\mathrm{H}}=6.5 \mathrm{~Hz}, 3 \mathrm{H}, \mathrm{CH}_{3}\right)$.

${ }^{13} \mathrm{C}-\left\{{ }^{1} \mathrm{H}\right\}$ NMR $\left(100 \mathrm{MHz}, \mathbf{C D C l}_{3}, \mathbf{2 9 8 K}\right): \delta(\mathrm{ppm})=144.6\left(\mathrm{~s}, \mathrm{C}^{\mathrm{IV}}\right), 133.4\left(\mathrm{~s}, \mathrm{C}^{\mathrm{IV}}\right), 129.0\left(\mathrm{~s}, C_{\mathrm{Ar}} \mathrm{H}\right), 127.1\left(\mathrm{~s}, C_{\mathrm{Ar}} \mathrm{H}\right)$, $70.1(\mathrm{~s}, \mathrm{CH}), 25.6\left(\mathrm{~s}, \mathrm{CH}_{3}\right)$.

1-(4-Methoxyphenyl)ethan-1-ol, $5 \mathbf{f}^{[3]}$<smiles>COc1ccc(C(C)O)cc1</smiles>

The general procedure yielded the title compound as a colourless oil (149 mg, 98\%).

${ }^{1} \mathbf{H}$ NMR (500 MHz, $\mathbf{C D C l}_{\mathbf{3}}, \mathbf{2 9 8 K}$, TMS): $\delta(\mathrm{ppm})=7.30\left(\mathrm{~d},{ }^{3} J_{\mathrm{H}-\mathrm{H}}=8.6 \mathrm{~Hz}, 2 \mathrm{H}, \mathrm{C}_{\mathrm{Ar}} \mathrm{H}\right), 6.89\left(\mathrm{~d},{ }^{3} J_{\mathrm{H}-\mathrm{H}}=8.6 \mathrm{~Hz}, 2 \mathrm{H}\right.$, $\left.\mathrm{C}_{\mathrm{Ar}} \mathrm{H}\right), 4.85\left(\mathrm{q},{ }^{3} J_{\mathrm{H}-\mathrm{H}}=6.5 \mathrm{~Hz}, 1 \mathrm{H}, \mathrm{CH}\right), 3.80\left(\mathrm{~s}, 3 \mathrm{H}, \mathrm{OCH}_{3}\right), 1.83(\mathrm{br} \mathrm{s}, 1 \mathrm{H}, \mathrm{OH}), 1.48\left(\mathrm{~d},{ }^{3} J_{\mathrm{H}-\mathrm{H}}=6.5 \mathrm{~Hz}, 3 \mathrm{H}, \mathrm{CH}_{3}\right)$.

${ }^{13} \mathrm{C}-\left\{{ }^{1} \mathrm{H}\right\}$ NMR (126 MHz, $\left.\mathbf{C D C l}_{3}, \mathbf{2 9 8 K}\right): \delta(\mathrm{ppm})=159.3\left(\mathrm{~s}, \mathrm{C}^{\mathrm{IV}}\right), 138.3\left(\mathrm{~s}, \mathrm{C}^{\mathrm{IV}}\right), 127.0\left(\mathrm{~s}, \mathrm{C}_{\mathrm{Ar}} \mathrm{H}\right), 114.1\left(\mathrm{~s}, \mathrm{C}_{\mathrm{Ar}} \mathrm{H}\right)$, $70.3(\mathrm{~s}, \mathrm{CH}), 55.6\left(\mathrm{~s}, \mathrm{OCH}_{3}\right), 25.3\left(\mathrm{~s}, \mathrm{CH}_{3}\right)$.

1-(o-Tolyl)ethan-1-ol, $\mathbf{5 g}^{[4]}$<smiles>Cc1ccccc1C(C)O</smiles>

The general procedure yielded the title compound as a colourless oil (135 mg, 99\%).

${ }^{1} \mathbf{H}$ NMR (500 MHz, $\left.\mathbf{C D C l}_{3}, \mathbf{2 9 8 K}, \mathbf{T M S}\right): \delta(\mathrm{ppm})=7.51\left(\mathrm{~d},{ }^{3} J_{\mathrm{H}-\mathrm{H}}=7.8 \mathrm{~Hz}, 1 \mathrm{H}, \mathrm{C}_{\mathrm{Ar}} \mathrm{H}\right), 7.23\left(\mathrm{t},{ }^{3} J_{\mathrm{H}-\mathrm{H}}=7.6 \mathrm{~Hz}, 1 \mathrm{H}\right.$, $\left.\mathrm{C}_{\mathrm{Ar}} \mathrm{H}\right), 7.17\left(\mathrm{t},{ }^{3} J_{\mathrm{H}-\mathrm{H}}=7.6 \mathrm{~Hz}, 1 \mathrm{H}, \mathrm{C}_{\mathrm{Ar}} \mathrm{H}\right), 7.13\left(\mathrm{~d},{ }^{3} J_{\mathrm{H}-\mathrm{H}}=7.8 \mathrm{~Hz}, 1 \mathrm{H}, \mathrm{C}_{\mathrm{Ar}} \mathrm{H}\right), 5.13\left(\mathrm{q},{ }^{3} J_{\mathrm{H}-\mathrm{H}}=6.5 \mathrm{~Hz}, 1 \mathrm{H}, \mathrm{CH}\right), 2.34(\mathrm{~s}$, $\left.3 \mathrm{H}, \mathrm{CH}_{3}\right), 1.76$ (br. s, $\left.1 \mathrm{H}, \mathrm{OH}\right), 1.47\left(\mathrm{~d},{ }^{3} \mathrm{~J}_{\mathrm{H}-\mathrm{H}}=6.5 \mathrm{~Hz}, 3 \mathrm{H}, \mathrm{CH}_{3}\right)$.

${ }^{13} \mathrm{C}-\left\{{ }^{1} \mathrm{H}\right\}$ NMR (126 MHz, $\left.\mathbf{C D C l}_{3}, \mathbf{2 9 8 K}\right): \delta(\mathrm{ppm})=144.2\left(\mathrm{~s}, \mathrm{C}^{\mathrm{IV}}\right), 134.6\left(\mathrm{~s}, \mathrm{C}^{\mathrm{IV}}\right), 130.7\left(\mathrm{~s}, \mathrm{C}_{\mathrm{Ar}} \mathrm{H}\right), 127.5\left(\mathrm{~s}, \mathrm{C}_{\mathrm{Ar}} \mathrm{H}\right)$, $126.7\left(\mathrm{~s}, \mathrm{C}_{\mathrm{Ar}} \mathrm{H}\right), 124.8\left(\mathrm{~s}, \mathrm{C}_{\mathrm{Ar}} \mathrm{H}\right), 67.2(\mathrm{~s}, \mathrm{CH}), 24.3\left(\mathrm{~s}, \mathrm{CH}_{3}\right), 19.3\left(\mathrm{~s}, \mathrm{CH}_{3}\right)$.

1-(6-Methoxynaphthalen-2-yl)ethan-1-ol, $5 h^{[5]}$<smiles>COc1ccc2cc(C(C)O)ccc2c1</smiles>

The general procedure yielded the title compound as a colourless solid (202 mg, 99\%).

${ }^{1} \mathrm{H}$ NMR (500 MHz, CDCl 3 , 298K, TMS): $\delta(\mathrm{ppm})=7.74\left(\mathrm{~m}, 3 \mathrm{H}, \mathrm{C}_{\mathrm{Ar}} \mathrm{H}\right), 7.47\left(\mathrm{dd},{ }^{3} J_{\mathrm{H}-\mathrm{H}}=8.5 \mathrm{~Hz},{ }^{5} J_{\mathrm{HH}}=1.5 \mathrm{~Hz}, 1 \mathrm{H}\right.$, $\left.\mathrm{C}_{\mathrm{Ar}} \mathrm{H}\right), 7.16-7.12\left(\mathrm{~m}, 2 \mathrm{H}, \mathrm{C}_{\mathrm{Ar}} \mathrm{H}\right), 5.04\left(\mathrm{q},{ }^{3} J_{\mathrm{H}-\mathrm{H}}=6.5 \mathrm{~Hz}, 1 \mathrm{H}, \mathrm{CH}\right), 3.92\left(\mathrm{~s}, 3 \mathrm{H}, \mathrm{OCH}_{3}\right), 1.88(\mathrm{br} \mathrm{s}, 1 \mathrm{H}, \mathrm{OH}), 1.57\left(\mathrm{~d},{ }^{3} J_{\mathrm{H}-}\right.$ $\mathrm{H}=6.5 \mathrm{~Hz}, 3 \mathrm{H}, \mathrm{CH}_{3}$ ).

${ }^{13} \mathrm{C}-\left\{{ }^{1} \mathrm{H}\right\}$ NMR (126 MHz, $\left.\mathbf{C D C l}_{3}, \mathbf{2 9 8 K}\right): \delta(\mathrm{ppm})=158.0\left(\mathrm{~s}, \mathrm{C}^{\mathrm{IV}}\right), 141.2\left(\mathrm{~s}, \mathrm{C}^{\mathrm{IV}}\right), 134.4\left(\mathrm{~s}, \mathrm{C}^{\mathrm{IV}}\right), 129.7\left(\mathrm{~s}, \mathrm{C}_{\mathrm{Ar}} \mathrm{H}\right)$, $129.1\left(\mathrm{~s}, \mathrm{C}^{\mathrm{IV}}\right), 127.5\left(\mathrm{~s}, \mathrm{C}_{\mathrm{Ar}} \mathrm{H}\right), 124.7\left(\mathrm{~s}, \mathrm{C}_{\mathrm{Ar}} \mathrm{H}\right), 124.1\left(\mathrm{~s}, \mathrm{C}_{\mathrm{Ar}} \mathrm{H}\right), 119.3\left(\mathrm{~s}, \mathrm{C}_{\mathrm{Ar}} \mathrm{H}\right), 106.0\left(\mathrm{~s}, \mathrm{C}_{\mathrm{Ar}} \mathrm{H}\right), 70.9(\mathrm{~s}, \mathrm{CH}), 55.6(\mathrm{~s}$, $\left.\mathrm{OCH}_{3}\right), 25.4\left(\mathrm{~s}, \mathrm{CH}_{3}\right)$. 
<smiles>CC(C)(C)C(O)c1ccccc1</smiles>

The general procedure yielded the title compound as a colourless solid (151 mg, 92\%).

${ }^{1}$ H NMR (500 MHz, CDCl 3 , 298K, TMS): $\delta(\mathrm{ppm})=7.33-7.24\left(\mathrm{~m}, 5 \mathrm{H}, \mathrm{C}_{\mathrm{Ar}} \mathrm{H}\right), 4.40(\mathrm{~s}, 1 \mathrm{H}, \mathrm{CH}), 1.86(\mathrm{~s}, 1 \mathrm{H}, \mathrm{OH})$, $0.92\left(\mathrm{~s}, 9 \mathrm{H}, \mathrm{CH}_{3}\right)$.

${ }^{13} \mathbf{C}-\left\{{ }^{1} \mathbf{H}\right\}$ NMR (126 MHz, $\left.\mathbf{C D C l}_{3}, \mathbf{2 9 8 K}\right): \delta(\mathrm{ppm})=142.5\left(\mathrm{~s}, \mathrm{C}^{\mathrm{IV}}\right), 127.9\left(\mathrm{~s}, \mathrm{C}_{\mathrm{Ar}} \mathrm{H}\right), 127.9\left(\mathrm{~s}, \mathrm{C}_{\mathrm{Ar}} \mathrm{H}\right), 127.6\left(\mathrm{~s}, \mathrm{C}_{\mathrm{Ar}} \mathrm{H}\right)$, $82.7(\mathrm{~s}, \mathrm{CH}), 36.0\left(\mathrm{~s}, \mathrm{C}^{\mathrm{IV}}\right), 26.2\left(\mathrm{~s}, \mathrm{CH}_{3}\right)$.

Diphenylmethanol, $\mathbf{5 j}^{[7]}$<smiles>OC(c1ccccc1)c1ccccc1</smiles>

The general procedure yielded the title compound as a colourless solid (178 mg, 97\%).

${ }^{1}$ H NMR (500 MHz, CDCl 3 , 298K, TMS): $\delta(\mathrm{ppm})=7.40-7.37\left(\mathrm{~m}, 4 \mathrm{H}, \mathrm{C}_{\mathrm{Ar}} \mathrm{H}\right), 7.36-7.32\left(\mathrm{~m}, 4 \mathrm{H}, \mathrm{C}_{\mathrm{Ar}} \mathrm{H}\right), 7.28-7.25(\mathrm{~m}$, $\left.2 \mathrm{H}, \mathrm{C}_{\mathrm{Ar}} \mathrm{H}\right), 5.85\left(\mathrm{~d},{ }^{3} J_{\mathrm{H}-\mathrm{H}}=3.0 \mathrm{~Hz}, 1 \mathrm{H}, \mathrm{CH}\right), 2.21\left(\mathrm{~d},{ }^{3} J_{\mathrm{H}-\mathrm{H}}=3.4 \mathrm{~Hz}, 1 \mathrm{H}, \mathrm{OH}\right)$.

${ }^{13} \mathbf{C}-\left\{{ }^{1} \mathrm{H}\right\}$ NMR (126 MHz, $\left.\mathbf{C D C l}_{3}, \mathbf{2 9 8 K}\right): \delta(\mathrm{ppm})=144.1\left(\mathrm{~s}, \mathrm{C}^{\mathrm{IV}}\right), 128.8\left(\mathrm{~s}, \mathrm{C}_{\mathrm{Ar}} \mathrm{H}\right), 127.9\left(\mathrm{~s}, \mathrm{C}_{\mathrm{Ar}} \mathrm{H}\right), 126.9\left(\mathrm{~s}, \mathrm{C}_{\mathrm{Ar}} \mathrm{H}\right)$, $76.6(\mathrm{~s}, \mathrm{CH})$.

Bis(4-methoxyphenyl)methanol, $5 \mathbf{k}^{[8]}$<smiles>COc1ccc(C(O)c2ccc(OC)cc2)cc1</smiles>

The general procedure yielded the title compound as a colourless solid (239 mg, 98\%).

${ }^{1}$ H NMR (500 MHz, CDCl 3 , 298K, TMS): $\delta(\mathrm{ppm})=7.30-7.26\left(\mathrm{~m}, 4 \mathrm{H}, \mathrm{C}_{\mathrm{Ar}} \mathrm{H}\right), 6.88-6.84\left(\mathrm{~m}, 4 \mathrm{H}, \mathrm{C}_{\mathrm{Ar}} \mathrm{H}\right), 5.77\left(\mathrm{~d},{ }^{3} J_{\mathrm{H}-\mathrm{H}}\right.$ $=3.5 \mathrm{~Hz}, 1 \mathrm{H}, \mathrm{CH}), 3.79\left(\mathrm{~s}, 6 \mathrm{H}, \mathrm{OCH}_{3}\right), 2.10\left(\mathrm{~d},{ }^{3} J_{\mathrm{H}-\mathrm{H}}=3.5 \mathrm{~Hz}, 1 \mathrm{H}, \mathrm{OH}\right)$.

${ }^{13} \mathbf{C}-\left\{{ }^{1} \mathbf{H}\right\}$ NMR (126 MHz, $\left.\mathbf{C D C l}_{3}, \mathbf{2 9 8 K}\right): \delta(\mathrm{ppm})=159.3\left(\mathrm{~s}, \mathrm{C}^{\mathrm{IV}}\right), 136.7\left(\mathrm{~s}, \mathrm{C}^{\mathrm{IV}}\right), 128.1\left(\mathrm{~s}, \mathrm{C}_{\mathrm{Ar}} \mathrm{H}\right), 114.1\left(\mathrm{~s}, \mathrm{C}_{\mathrm{Ar}} \mathrm{H}\right)$, $75.7(\mathrm{~s}, \mathrm{CH}), 55.6\left(\mathrm{~s}, \mathrm{OCH}_{3}\right)$

10,11-Dihydro-5H-dibenzo[a,d][7] annulen-5-ol, $5 \mathrm{I}^{[9]}$<smiles>OC1c2ccccc2CCc2ccccc21</smiles>

The general procedure yielded the title compound as a colourless solid (208 mg, 99\%). 
${ }^{1} \mathbf{H}$ NMR (500 MHz, $\mathbf{C D C l}_{3}, \mathbf{2 9 8 K}$, TMS): $\delta(\mathrm{ppm})=7.46-7.43\left(\mathrm{~m}, 2 \mathrm{H}, \mathrm{C}_{\mathrm{Ar}} \mathrm{H}\right), 7.22-7.13\left(\mathrm{~m}, 6 \mathrm{H}, \mathrm{C}_{\mathrm{Ar}} \mathrm{H}\right), 5.96\left(\mathrm{~d},{ }^{4} J_{\mathrm{HH}}\right.$ $=2.6 \mathrm{~Hz}, 1 \mathrm{H}, \mathrm{CH}), 3.47-3.39\left(\mathrm{~m}, 2 \mathrm{H}, \mathrm{CH}_{2}\right), 3.15-3.07\left(\mathrm{~m}, 2 \mathrm{H}, \mathrm{CH}_{2}\right), 2.23\left(\mathrm{~d},{ }^{5} J_{\mathrm{HH}}=2.9 \mathrm{~Hz}, 1 \mathrm{H}, \mathrm{OH}\right)$.

${ }^{13} \mathrm{C}-\left\{{ }^{1} \mathrm{H}\right\}$ NMR $\left(126 \mathrm{MHz}, \mathbf{C D C l}_{\mathbf{3}}, \mathbf{2 9 8 K}\right): \delta(\mathrm{ppm})=140.8\left(\mathrm{~s}, \mathrm{C}^{\mathrm{IV}}\right), 139.2\left(\mathrm{~s}, \mathrm{C}^{\mathrm{IV}}\right), 130.5\left(\mathrm{~s}, C_{\mathrm{Ar}} \mathrm{H}\right), 128.3\left(\mathrm{~s}, C_{\mathrm{Ar}} \mathrm{H}\right)$, $127.4\left(\mathrm{~s}, C_{\mathrm{Ar}} \mathrm{H}\right), 126.5\left(\mathrm{~s}, C_{\mathrm{Ar}} \mathrm{H}\right), 76.9(\mathrm{~s}, \mathrm{CH}), 32.7\left(\mathrm{~s}, \mathrm{CH}_{2}\right)$.

2,3-Dihydro-1 $H$-inden-1-ol, $5 \mathrm{~m}^{[10]}$<smiles>OC1CCc2ccccc21</smiles>

The general procedure yielded the title compound as a colourless solid (131 mg, 98\%).

${ }^{1} \mathrm{H}$ NMR (500 MHz, $\mathbf{C D C l}_{3}, \mathbf{2 9 8 K}$, TMS): $\delta(\mathrm{ppm})=7.42-7.39\left(\mathrm{~m}, 1 \mathrm{H}, \mathrm{C}_{\mathrm{Ar}} \mathrm{H}\right), 7.27-7.21\left(\mathrm{~m}, 3 \mathrm{H}, \mathrm{C}_{\mathrm{Ar}} \mathrm{H}\right), 5.24\left(\mathrm{t},{ }^{3} J_{\mathrm{H}-\mathrm{H}}\right.$ $=6.0 \mathrm{~Hz}, 1 \mathrm{H}, \mathrm{CH}), 3.09-3.02\left(\mathrm{~m}, 1 \mathrm{H}, \mathrm{CH}_{2}\right), 2.85-2.78\left(\mathrm{~m}, 1 \mathrm{H}, \mathrm{CH}_{2}\right), 2.52-2.44\left(\mathrm{~m}, 1 \mathrm{H}, \mathrm{CH}_{2}\right), 1.98-1.90\left(\mathrm{~m}, 1 \mathrm{H}, \mathrm{CH}_{2}\right)$, 1.84 (br. s, $1 \mathrm{H}, \mathrm{OH}$ ).

${ }^{13} \mathrm{C}-\left\{{ }^{1} \mathrm{H}\right\}$ NMR (126 MHz, $\left.\mathbf{C D C l}_{3}, \mathbf{2 9 8 K}\right): \delta(\mathrm{ppm})=145.3\left(\mathrm{~s}, \mathrm{C}^{\mathrm{IV}}\right), 143.6\left(\mathrm{~s}, \mathrm{C}^{\mathrm{IV}}\right), 128.6\left(\mathrm{~s}, C_{\mathrm{Ar}} \mathrm{H}\right), 127.0\left(\mathrm{~s}, C_{\mathrm{Ar}} \mathrm{H}\right)$, $125.2\left(\mathrm{~s}, C_{\mathrm{Ar}} \mathrm{H}\right), 124.5\left(\mathrm{~s}, C_{\mathrm{Ar}} \mathrm{H}\right), 76.8(\mathrm{~s}, \mathrm{CH}), 36.3\left(\mathrm{~s}, \mathrm{CH}_{2}\right), 30.1\left(\mathrm{~s}, \mathrm{CH}_{2}\right)$.

1-(Pyridin-2-yl)ethan-1-ol, $5 \mathbf{n}^{[4]}$<smiles>CC(O)c1ccccn1</smiles>

The general procedure yielded the title compound as a colourless oil (109 mg, 89\%).

${ }^{1} \mathrm{H}$ NMR (500 MHz, $\mathbf{C D C l}_{3}$, 298K, TMS): $\delta(\mathrm{ppm})=8.53\left(\mathrm{~d},{ }^{3} J_{\mathrm{H}-\mathrm{H}}=4.0 \mathrm{~Hz}, 1 \mathrm{H}, \mathrm{C}_{\mathrm{Ar}} \mathrm{H}\right), 7.69\left(\mathrm{td},{ }^{3} J_{\mathrm{H}-\mathrm{H}}=7.6 \mathrm{~Hz},{ }^{4} J_{\mathrm{H}-\mathrm{H}}\right.$ $\left.=1.6 \mathrm{~Hz} 1 \mathrm{H}, \mathrm{C}_{\mathrm{Ar}} \mathrm{H}\right), 7.29\left(\mathrm{~d},{ }^{3} J_{\mathrm{H}-\mathrm{H}}=7.6 \mathrm{~Hz}, 1 \mathrm{H}, \mathrm{C}_{\mathrm{Ar}} \mathrm{H}\right), 7.22-7.18\left(\mathrm{~m}, 1 \mathrm{H}, \mathrm{C}_{\mathrm{Ar}} \mathrm{H}\right), 4.90\left(\mathrm{q},{ }^{3} J_{\mathrm{H}-\mathrm{H}}=6.5 \mathrm{~Hz}, 1 \mathrm{H}, \mathrm{CH}\right), 4.37$ (br s, $1 \mathrm{H}, \mathrm{OH}), 1.51\left(\mathrm{~d},{ }^{3} \mathrm{~J}_{\mathrm{H}-\mathrm{H}}=6.5 \mathrm{~Hz}, 3 \mathrm{H}, \mathrm{CH}_{3}\right)$.

${ }^{13} \mathrm{C}-\left\{{ }^{1} \mathrm{H}\right\}$ NMR $\left(126 \mathrm{MHz}, \mathbf{C D C l}_{3}, \mathbf{2 9 8 K}\right): \delta(\mathrm{ppm})=163.4\left(\mathrm{~s}, \mathrm{C}^{\mathrm{IV}}\right), 148.4\left(\mathrm{~s}, \mathrm{C}_{\mathrm{Ar}} \mathrm{H}\right), 137.1\left(\mathrm{~s}, \mathrm{C}_{\mathrm{Ar}} \mathrm{H}\right), 122.5\left(\mathrm{~s}, \mathrm{C}_{\mathrm{Ar}} \mathrm{H}\right)$, $120.1\left(\mathrm{~s}, \mathrm{C}_{\mathrm{Ar}} \mathrm{H}\right), 69.2(\mathrm{~s}, \mathrm{CH}), 24.5\left(\mathrm{~s}, \mathrm{CH}_{3}\right)$.

Nonan-5-ol, 50 ${ }^{[11]}$<smiles>CCCCC(O)CCCC</smiles>

The general procedure yielded the title compound as a colourless oil (131 mg, 91\%).

${ }^{1} \mathrm{H}$ NMR (500 MHz, $\mathbf{C D C l}_{3}, \mathbf{2 9 8 K}$, TMS): $\delta(\mathrm{ppm})=3.62-3.56(\mathrm{~m}, 1 \mathrm{H}, \mathrm{CH}), 1.51-1.26\left(\mathrm{~m}, 13 \mathrm{H}, \mathrm{CH}_{2}\right.$ and $\left.\mathrm{OH}\right), 0.91$ $\left(\mathrm{t},{ }^{3} \mathrm{~J}_{\mathrm{H}-\mathrm{H}}=6.9 \mathrm{~Hz}, 6 \mathrm{H}, \mathrm{CH}_{3}\right)$.

${ }^{13} \mathrm{C}-\left\{{ }^{1} \mathrm{H}\right\}$ NMR (126 MHz, $\left.\mathbf{C D C l}_{3}, \mathbf{2 9 8 K}\right): \delta(\mathrm{ppm})=72.3(\mathrm{~s}, \mathrm{CH}), 37.5\left(\mathrm{~s}, \mathrm{CH}_{2}\right), 28.2\left(\mathrm{~s}, \mathrm{CH}_{2}\right), 23.1\left(\mathrm{~s}, \mathrm{CH}_{2}\right), 14.4(\mathrm{~s}$, $\mathrm{CH}_{3}$ ). 
<smiles>CC(O)CCc1ccccc1</smiles>

The general procedure yielded the title compound as a colourless oil (150 mg, 99\%).

${ }^{1}$ H NMR (500 MHz, CDCl 3 , 298K, TMS): $\delta(\mathrm{ppm})=7.31-7.16\left(\mathrm{~m}, 5 \mathrm{H}, \mathrm{C}_{\mathrm{Ar}} \mathrm{H}\right), 3.82\left(\mathrm{sex},{ }^{3} J_{\mathrm{H}-\mathrm{H}}=6.2 \mathrm{~Hz}, 1 \mathrm{H}, \mathrm{CH}\right)$, 2.81-2.62 (m, 2H, $\left.\mathrm{CH}_{2}\right), 1.81-1.73\left(\mathrm{~m}, 2 \mathrm{H}, \mathrm{CH}_{2}\right), 1.45$ (br s, $\left.1 \mathrm{H}, \mathrm{OH}\right), 1.23\left(\mathrm{~d},{ }^{3} J_{\mathrm{H}-\mathrm{H}}=6.2 \mathrm{~Hz}, 3 \mathrm{H}, \mathrm{CH}_{3}\right)$.

${ }^{13} \mathrm{C}-\left\{{ }^{1} \mathrm{H}\right\}$ NMR (126 MHz, $\left.\mathbf{C D C l}_{3}, \mathbf{2 9 8 K}\right): \delta(\mathrm{ppm})=142.4\left(\mathrm{~s}, \mathrm{C}^{\mathrm{IV}}\right), 128.7\left(\mathrm{~s}, \mathrm{C}_{\mathrm{Ar}} \mathrm{H}\right), 126.1\left(\mathrm{~s}, \mathrm{C}_{\mathrm{Ar}} \mathrm{H}\right), 67.8(\mathrm{~s}, \mathrm{CH})$, $41.2\left(\mathrm{~s}, \mathrm{CH}_{2}\right), 32.5\left(\mathrm{~s}, \mathrm{CH}_{2}\right), 24.0\left(\mathrm{~s}, \mathrm{CH}_{3}\right)$.

\section{1-(Adamantan-2-yl)ethan-1-ol, 5q}<smiles>CC(O)C1C2CC3CC(C2)CC1C3</smiles>

The general procedure yielded the title compound as a colourless solid (178 $\mathrm{mg}, 99 \%)$.

${ }^{1}$ H NMR (500 MHz, CDCl 3 , 298K, TMS): $\delta(\mathrm{ppm})=3.29\left(\mathrm{q},{ }^{3} J_{\mathrm{H}-\mathrm{H}}=6.5 \mathrm{~Hz}, 1 \mathrm{H}, \mathrm{CH}\right), 2.02-1.46$ (m, $\left.15 \mathrm{H}\right), 1.29$ (br. s, $1 \mathrm{H}, \mathrm{OH}), 1.10\left(\mathrm{~d},{ }^{3} J_{\mathrm{H}-\mathrm{H}}=6.5 \mathrm{~Hz}, 1 \mathrm{H}, \mathrm{CH}_{3}\right)$.

${ }^{13} \mathrm{C}-\left\{{ }^{1} \mathbf{H}\right\}$ NMR $\left(126 \mathbf{M H z}, \mathbf{C D C l}_{3}, \mathbf{2 9 8 K}\right): \delta(\mathrm{ppm})=76.2(\mathrm{~s}, \mathrm{CH}), 38.1\left(\mathrm{~s}, \mathrm{CH}_{2}\right), 37.6\left(\mathrm{~s}, \mathrm{CH}_{2}\right), 28.7(\mathrm{~s}, \mathrm{CH}), 16.8(\mathrm{~s}$, $\left.\mathrm{CH}_{3}\right)$.

Adamantan-2-ol, 5r ${ }^{[13]}$<smiles>OC1C2CC3CC(C2)CC1C3</smiles>

The general procedure yielded the title compound as a colourless solid (123 mg, 81\%).

${ }^{1}$ H NMR (500 MHz, CDCl 3 , 298K, TMS): $\delta(\mathrm{ppm})=3.89-3.86(\mathrm{~m}, 1 \mathrm{H}, \mathrm{CH}), 2.10-1.20$ (m, 15H).

${ }^{13} \mathbf{C}-\left\{{ }^{1} \mathbf{H}\right\}$ NMR (126 MHz, $\left.\mathbf{C D C l}_{3}, \mathbf{2 9 8 K}\right): \delta(\mathrm{ppm})=74.9(\mathrm{~s}, \mathrm{CH}), 37.9\left(\mathrm{~s}, \mathrm{CH}_{2}\right), 36.8\left(\mathrm{~s}, \mathrm{CH}_{2}\right), 34.9(\mathrm{~s}, \mathrm{CH}), 31.4(\mathrm{~s}$, $\left.\mathrm{CH}_{2}\right), 27.8(\mathrm{~s}, \mathrm{CH}), 27.4(\mathrm{~s}, \mathrm{CH})$.

$3 \beta, 17 \beta-D i h y d r o x y a n d r o s t-5-e n e, 5 s^{[14]}$<smiles>C[C@H](O)[C@@]1(C)CC[C@H]2[C@@H]1CC=C1C[C@H](O)CC[C@@]12C</smiles> 
The general procedure yielded the title compound as a colourless solid (284 mg, 98\%).

${ }^{1} \mathbf{H}$ NMR (500 MHz, $\left.\mathbf{C D C l}_{3}, \mathbf{2 9 8 K}\right): \delta(\mathrm{ppm})=5.35\left(\mathrm{~d},{ }^{3} J_{\mathrm{H}-\mathrm{H}}=5.3 \mathrm{~Hz}, 1 \mathrm{H}, \mathrm{CH}\right), 3.65\left(\mathrm{t},{ }^{3} J_{\mathrm{H}-\mathrm{H}}=8.5 \mathrm{~Hz}, 1 \mathrm{H}, \mathrm{CH}\right), 3.57-$ $3.48(\mathrm{~m}, 1 \mathrm{H}, \mathrm{CH}), 2.34-2.20(\mathrm{~m}, 2 \mathrm{H}), 2.12-1.94(\mathrm{~m}, 2 \mathrm{H}), 1.89-1.80(\mathrm{~m}, 3 \mathrm{H}), 1.67-1.38(\mathrm{~m}, 10 \mathrm{H}), 1.34-1.23(\mathrm{~m}, 1 \mathrm{H})$, $1.14-0.90(\mathrm{~m}, 6 \mathrm{H}), 0.76(\mathrm{~s}, 3 \mathrm{H})$.

${ }^{13} \mathrm{C}-\left\{{ }^{1} \mathrm{H}\right\}$ NMR $\left(126 \mathrm{MHz}, \mathbf{C D C l}_{3}, \mathbf{2 9 8 K}\right): \delta(\mathrm{ppm})=141.2\left(\mathrm{~s}, \mathrm{C}^{\mathrm{IV}}\right), 121.7(\mathrm{~s}, \mathrm{CH}), 82.2(\mathrm{~s}, \mathrm{CH}), 72.0(\mathrm{~s}, \mathrm{CH}), 51.6(\mathrm{~s}$, $\mathrm{CH}), 50.5(\mathrm{~s}, \mathrm{CH}), 43.0\left(\mathrm{~s}, \mathrm{C}^{\mathrm{IV}}\right), 42.6\left(\mathrm{~s}, \mathrm{CH}_{2}\right), 37.6\left(\mathrm{~s}, \mathrm{CH}_{2}\right), 36.9\left(\mathrm{~s}, \mathrm{CH}_{2}\right), 32.3(\mathrm{~s}, \mathrm{CH}), 31.9\left(\mathrm{~s}, \mathrm{CH}_{2}\right), 31.8\left(\mathrm{~s}, \mathrm{CH}_{2}\right)$, $30.8\left(\mathrm{~s}, \mathrm{CH}_{2}\right), 23.8\left(\mathrm{~s}, \mathrm{CH}_{2}\right), 21.0\left(\mathrm{~s}, \mathrm{CH}_{2}\right), 19.7\left(\mathrm{~s}, \mathrm{CH}_{3}\right), 11.3\left(\mathrm{~s}, \mathrm{CH}_{3}\right)$. 
NMR spectra of $[\mathrm{Cu}(\mathrm{trz})(\mathrm{NHC})]_{2}$ complexes

${ }^{1} \mathrm{H}$ NMR (500 MHz, $\left.\mathrm{C}_{6} \mathrm{D}_{6}, 298 \mathrm{~K}\right)$ of $[\mathrm{Cu}(\mathrm{trz})(\mathrm{IPr})]_{2}, \mathbf{2 a}$

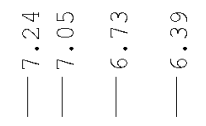

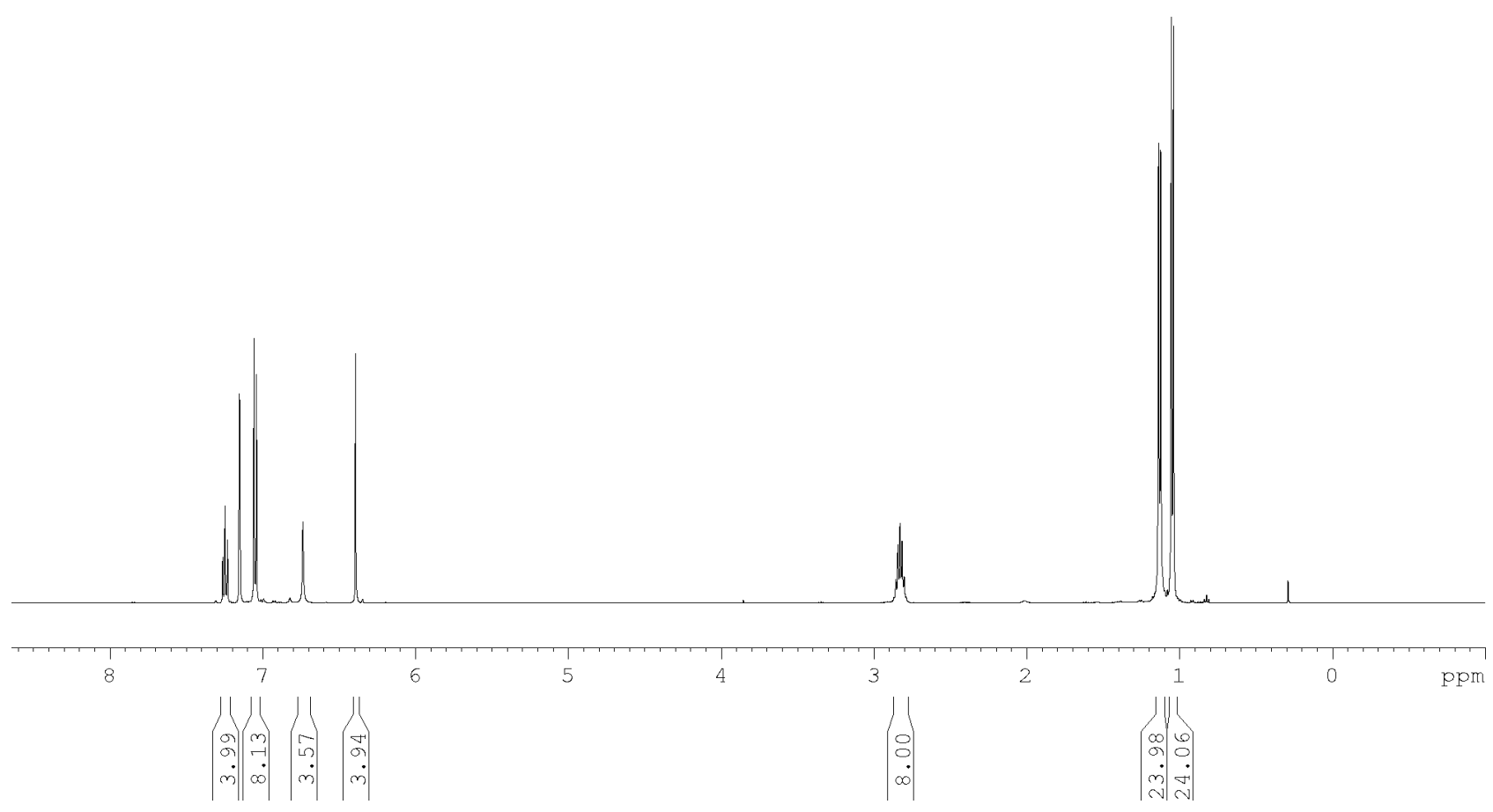

${ }^{13} \mathrm{C}-\left\{{ }^{1} \mathrm{H}\right\}$ NMR $\left(126 \mathrm{MHz}, \mathrm{C}_{6} \mathrm{D}_{6}, 298 \mathrm{~K}\right)$ of $[\mathrm{Cu}(\operatorname{trz})(\mathrm{IPr})]_{2}, \mathbf{2 a}$
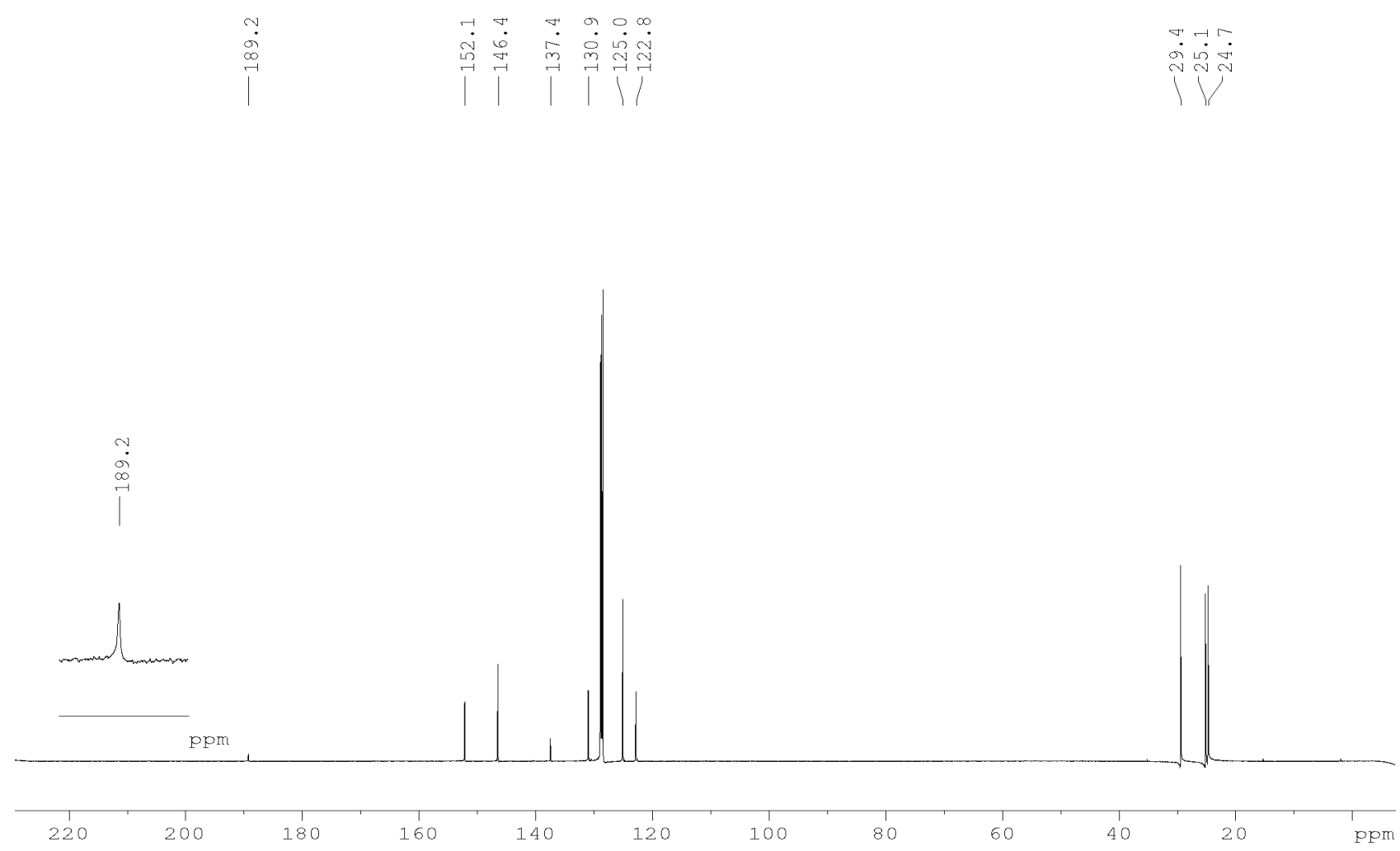
${ }^{1} \mathrm{H}$ NMR (400 MHz, $\left.\mathrm{CD}_{2} \mathrm{Cl}_{2}, 298 \mathrm{~K}\right)$ of $[\mathrm{Cu}(\mathrm{trz})(\mathrm{IMes})]_{2}, \mathbf{2 b}$

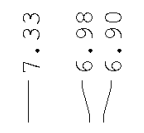

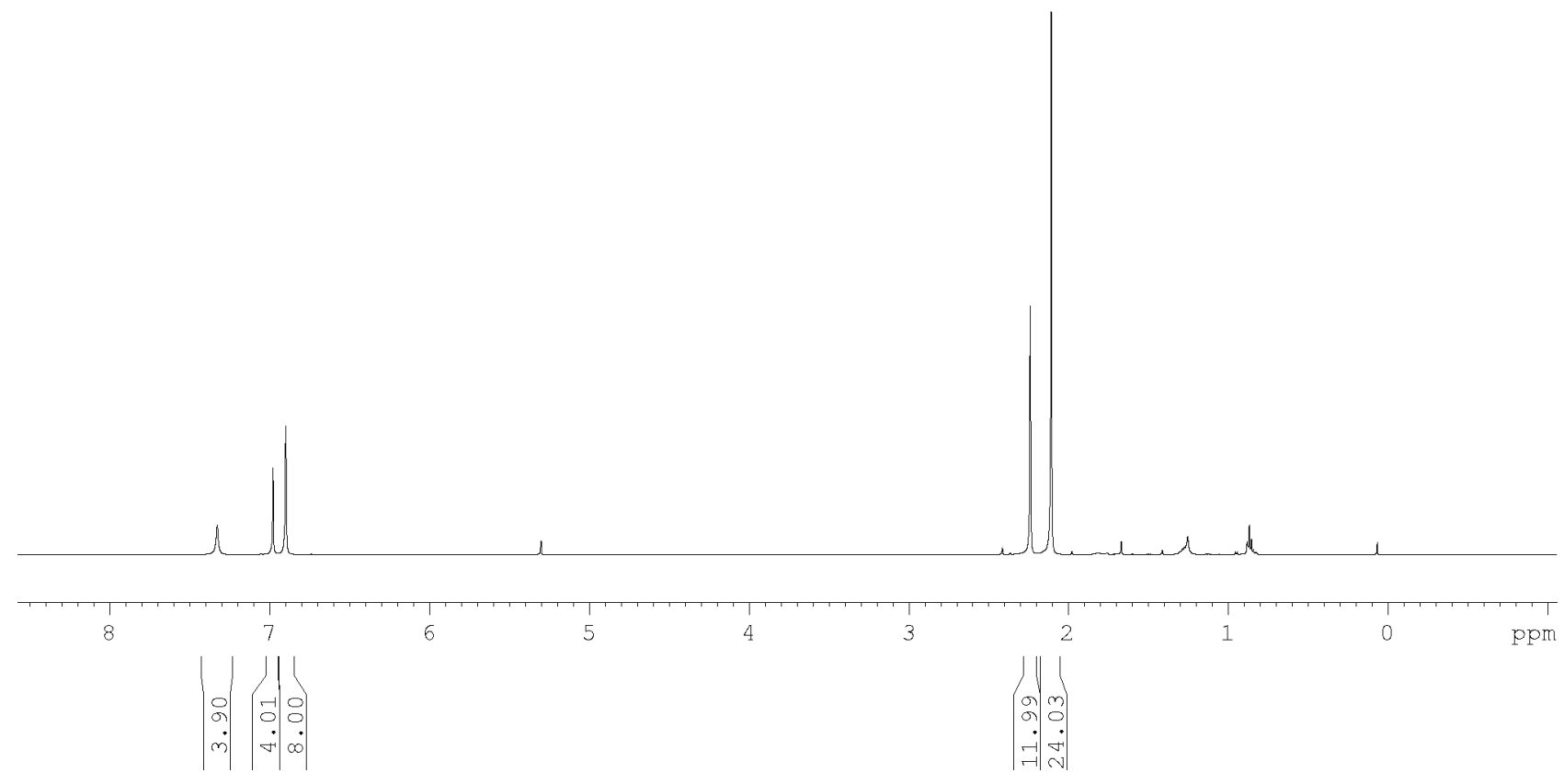

${ }^{13} \mathrm{C}-\left\{{ }^{1} \mathrm{H}\right\} \operatorname{NMR}\left(126 \mathrm{MHz}, \mathrm{CD}_{2} \mathrm{Cl}_{2}, 298 \mathrm{~K}\right)$ of $[\mathrm{Cu}(\mathrm{trz})(\mathrm{IMes})]_{2}, \mathbf{2 b}$
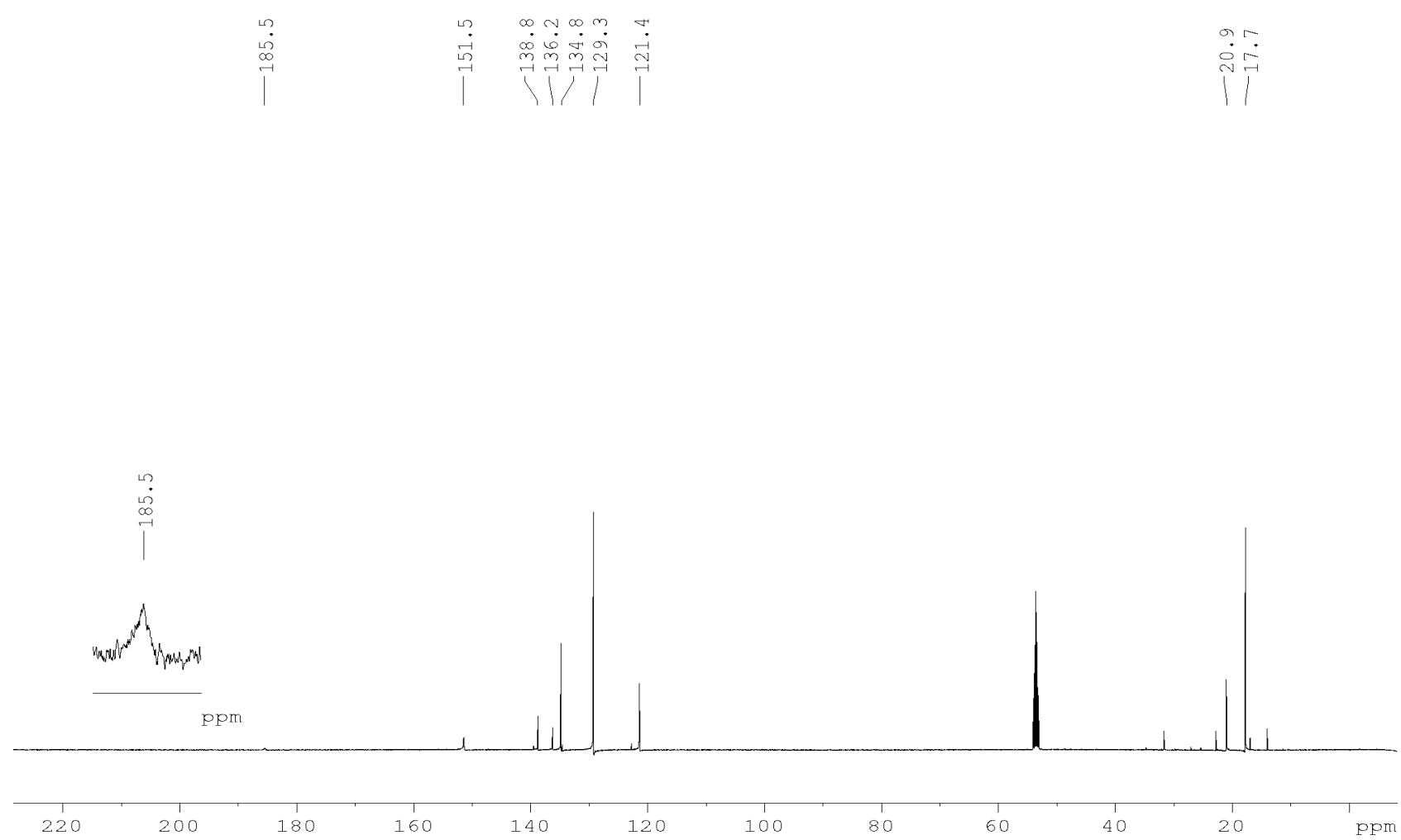
${ }^{1} \mathrm{H}$ NMR (500 MHz, $\left.\mathrm{CD}_{2} \mathrm{Cl}_{2}, 298 \mathrm{~K}\right)$ of $[\mathrm{Cu}(\mathrm{trz})(\mathrm{SIMes})]_{2}, \mathbf{2 c}$

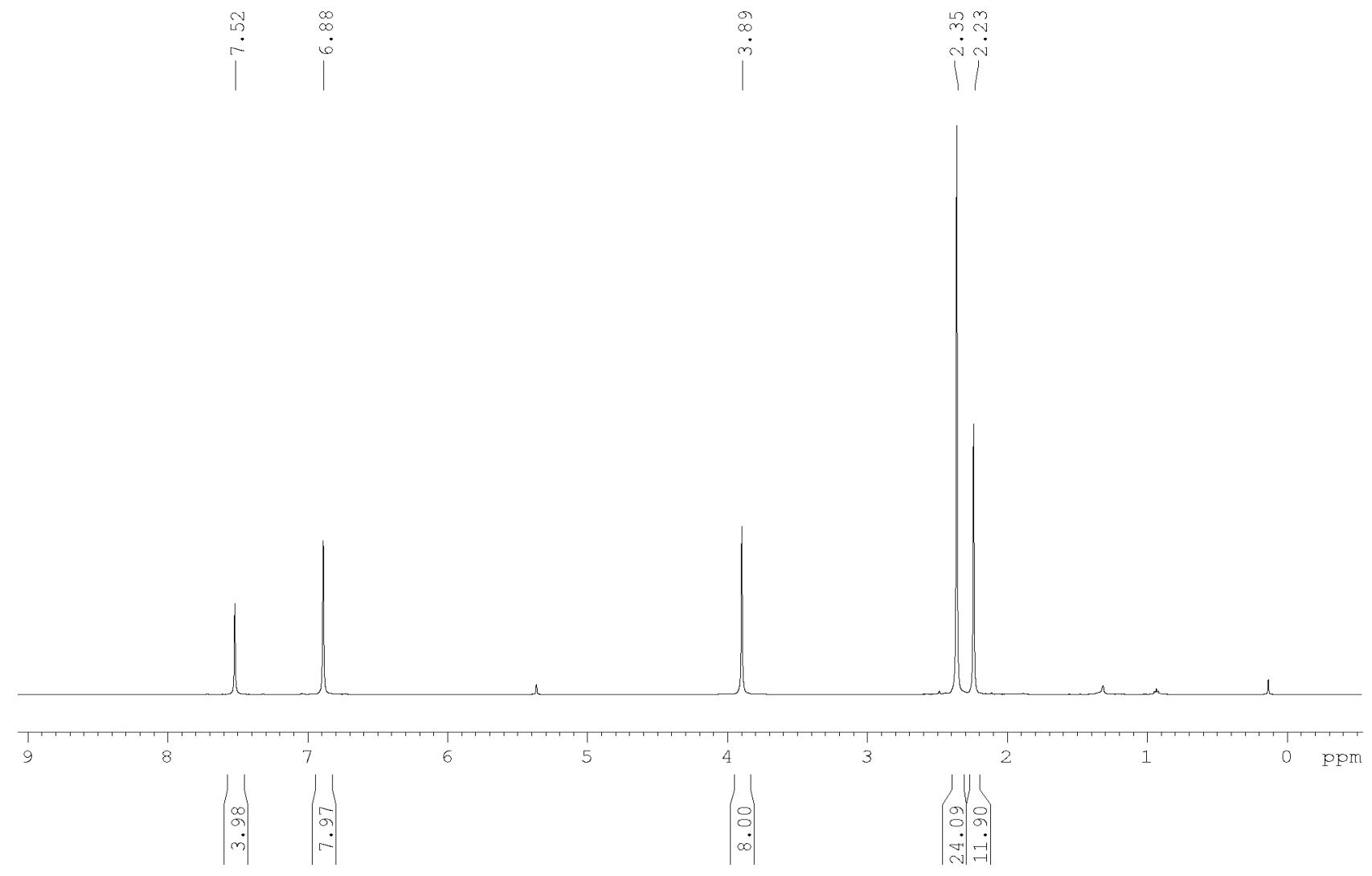

${ }^{13} \mathrm{C}-\left\{{ }^{1} \mathrm{H}\right\}$ NMR $\left(126 \mathrm{MHz}, \mathrm{CD}_{2} \mathrm{Cl}_{2}, 298 \mathrm{~K}\right)$ of $[\mathrm{Cu}(\operatorname{trz})(\mathrm{SIMes})]_{2}, \mathbf{2 c}$
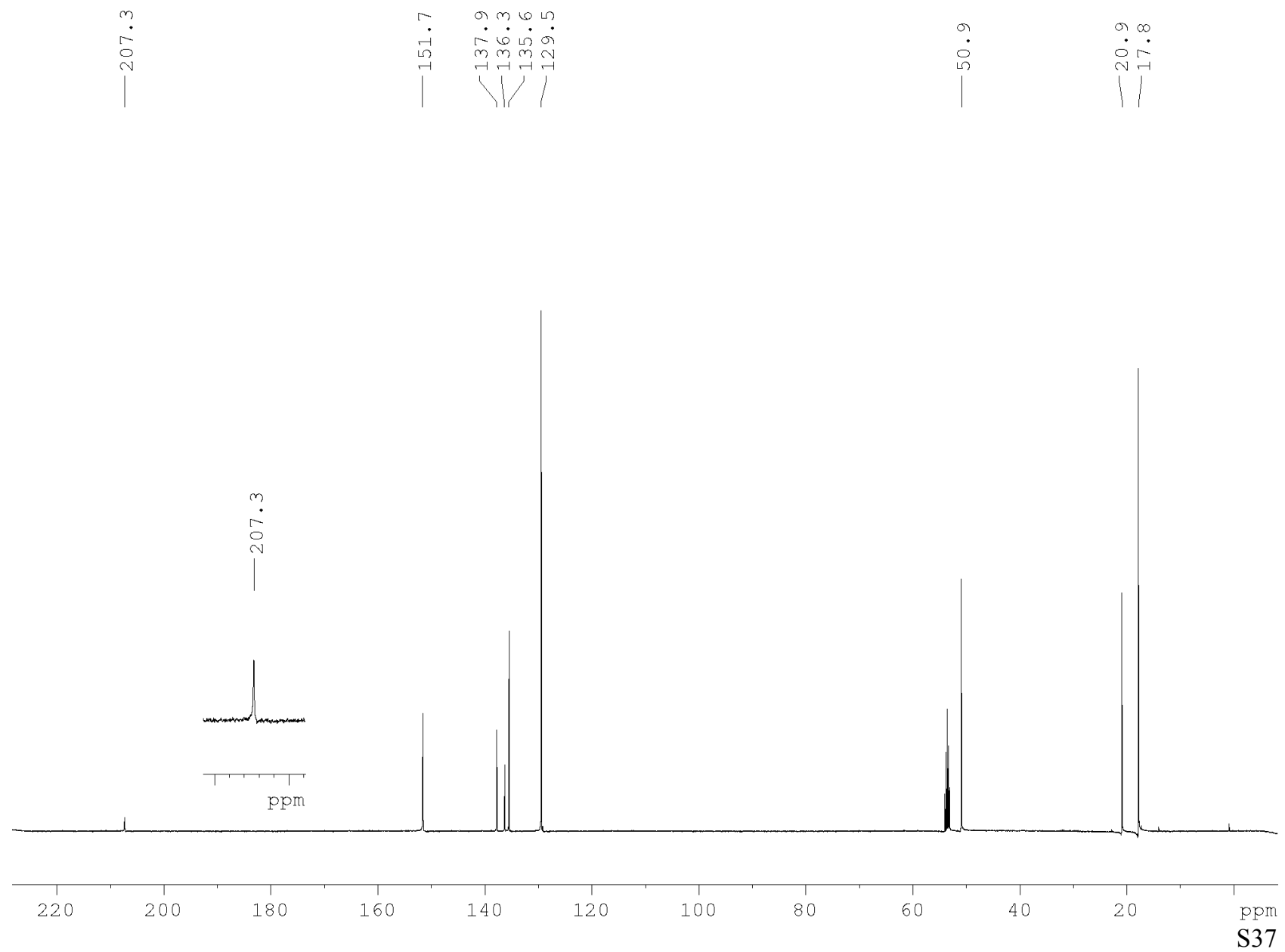
${ }^{1} \mathrm{H}$ NMR $\left(500 \mathrm{MHz}, \mathrm{CD}_{2} \mathrm{Cl}_{2}, 298 \mathrm{~K}\right)$ of $\left[\mathrm{Cu}(\operatorname{trz})\left(\mathrm{I}^{t} \mathrm{Bu}\right)\right]_{2}, \mathbf{2 d}$
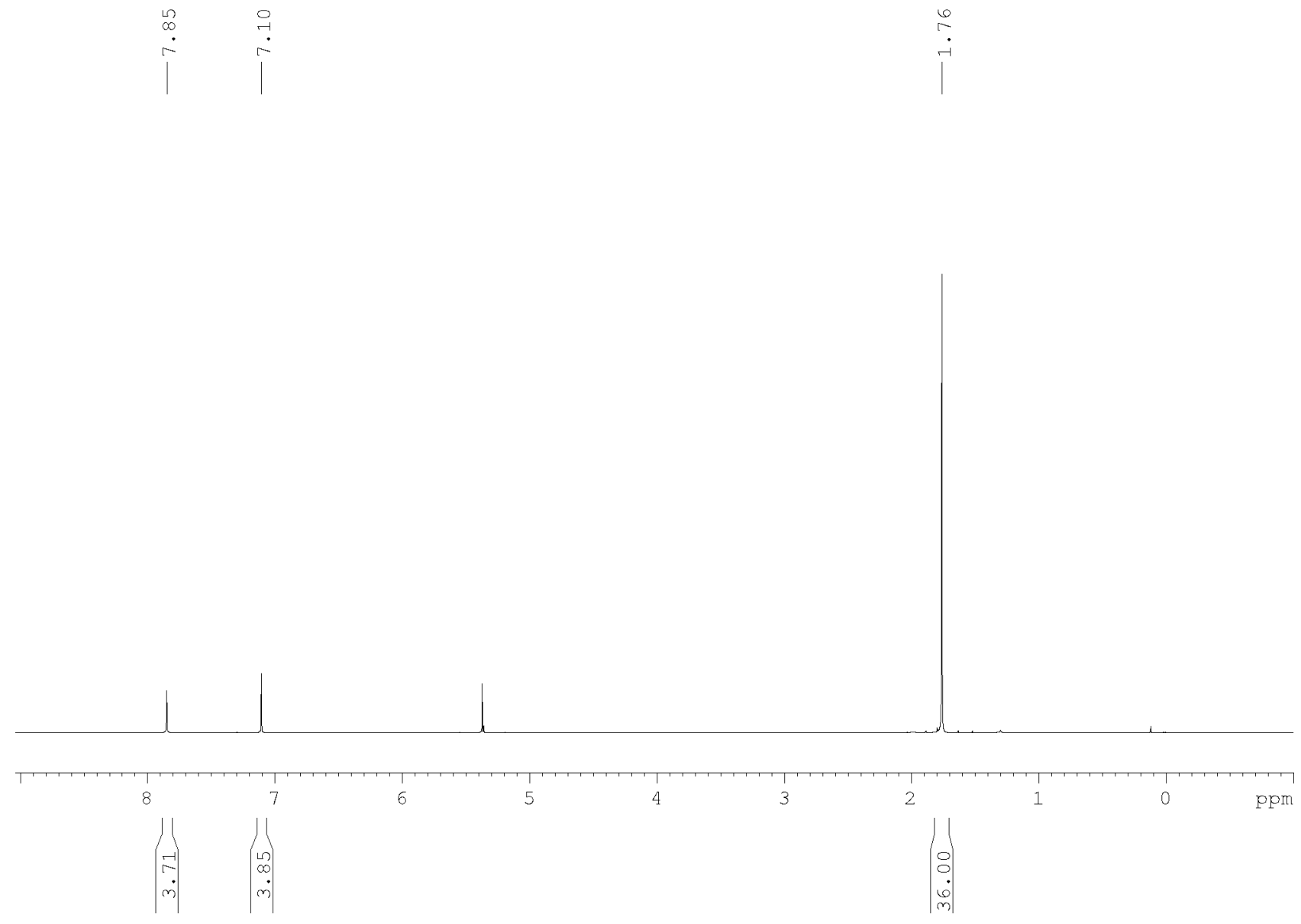

${ }^{13} \mathrm{C}-\left\{{ }^{1} \mathrm{H}\right\} \mathrm{NMR}\left(126 \mathrm{MHz}, \mathrm{CD}_{2} \mathrm{Cl}_{2}, 298 \mathrm{~K}\right)$ of $\left[\mathrm{Cu}(\operatorname{trz})\left(\mathrm{I}^{t} \mathrm{Bu}\right)\right]_{2}, \mathbf{2 d}$
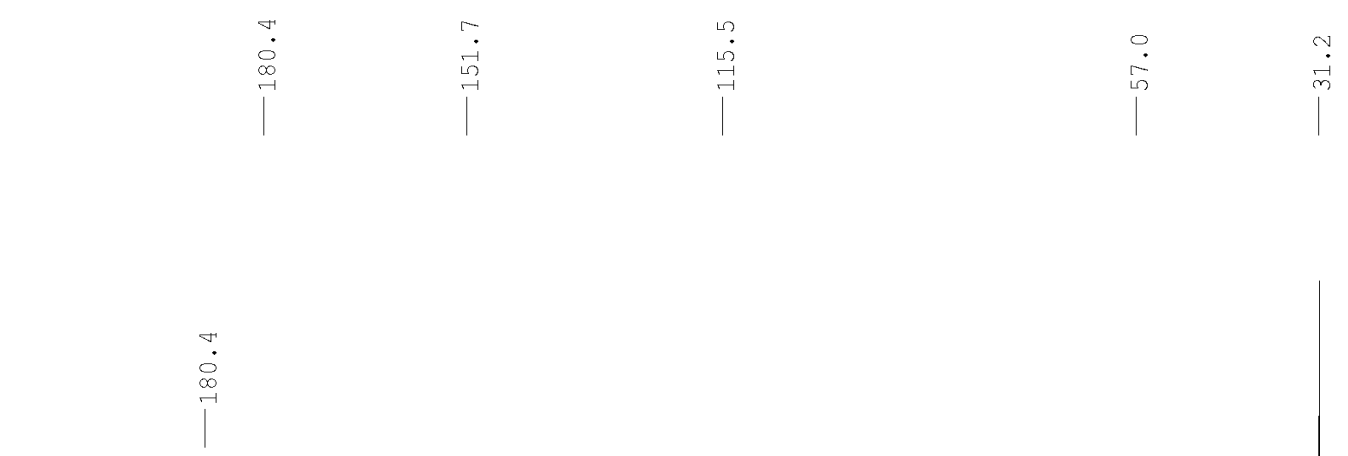

$\stackrel{i}{i}$

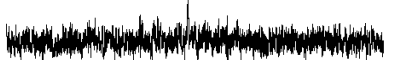

200190180170 pom

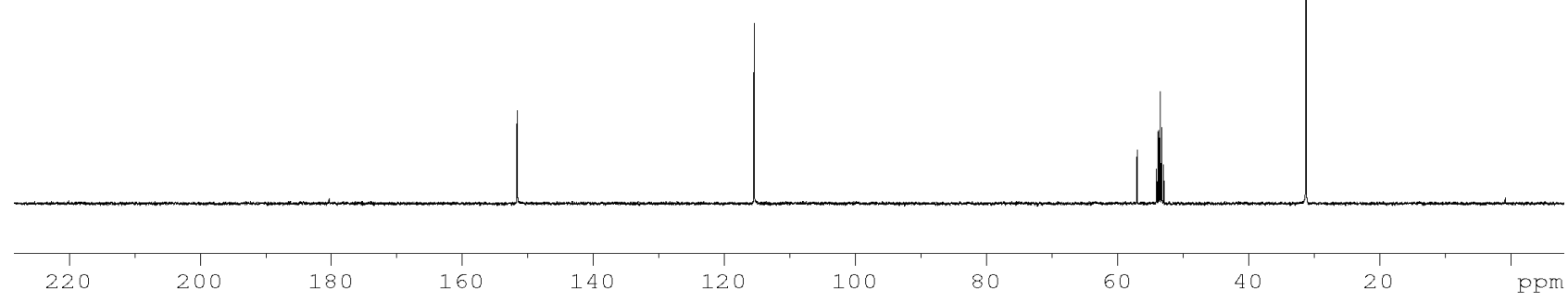


${ }^{1} \mathrm{H}$ NMR $\left(500 \mathrm{MHz}, \mathrm{CD}_{2} \mathrm{Cl}_{2}, 298 \mathrm{~K}\right)$ of $[\mathrm{Cu}(\operatorname{trz})(\mathrm{ICy})]_{2}, \mathbf{2 e}$
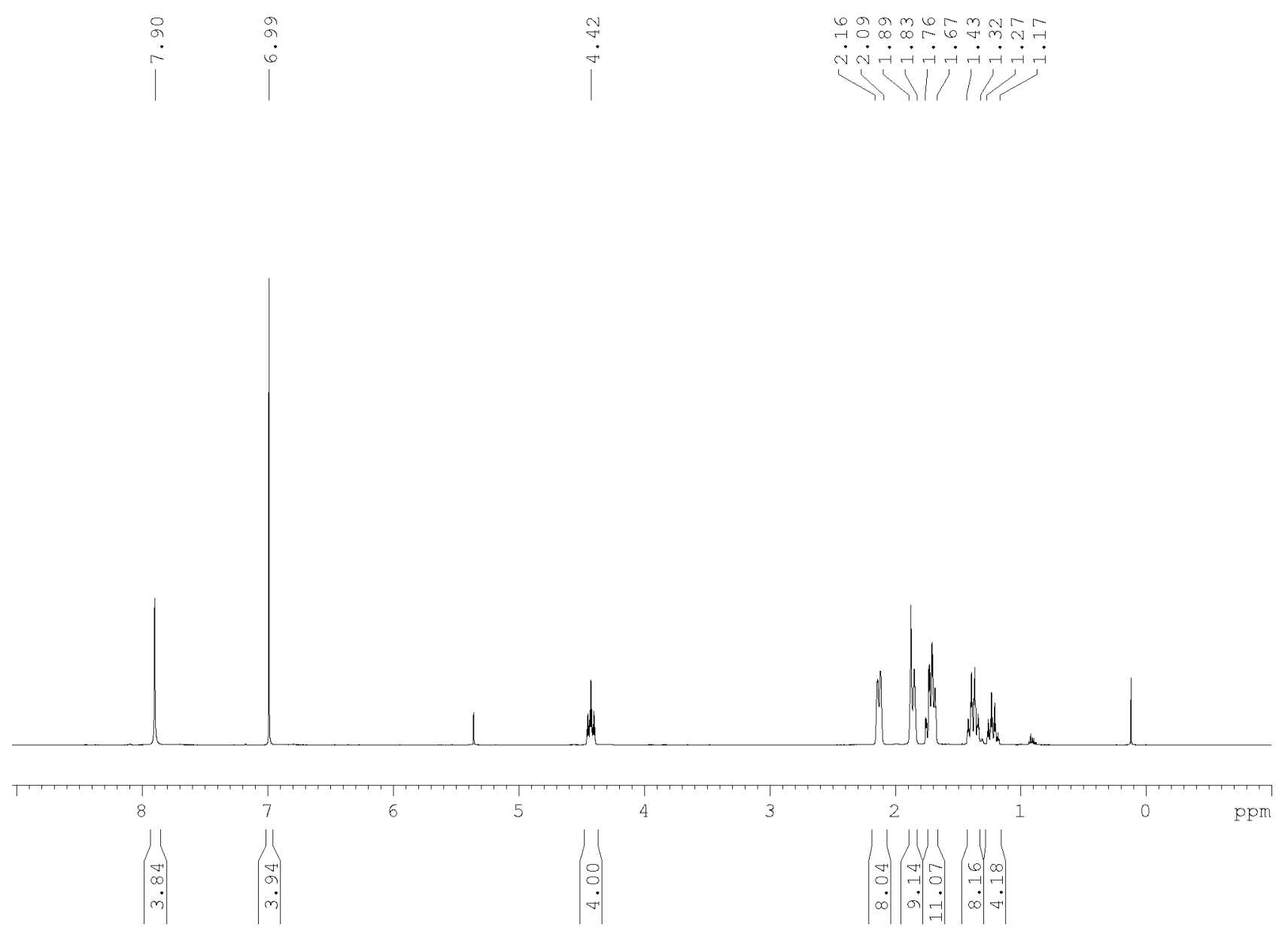

${ }^{13} \mathrm{C}-\left\{{ }^{1} \mathrm{H}\right\}$ NMR $\left(126 \mathrm{MHz}, \mathrm{CD}_{2} \mathrm{Cl}_{2}, 298 \mathrm{~K}\right)$ of $[\mathrm{Cu}(\operatorname{trz})(\mathrm{ICy})]_{2}, \mathbf{2 e}$

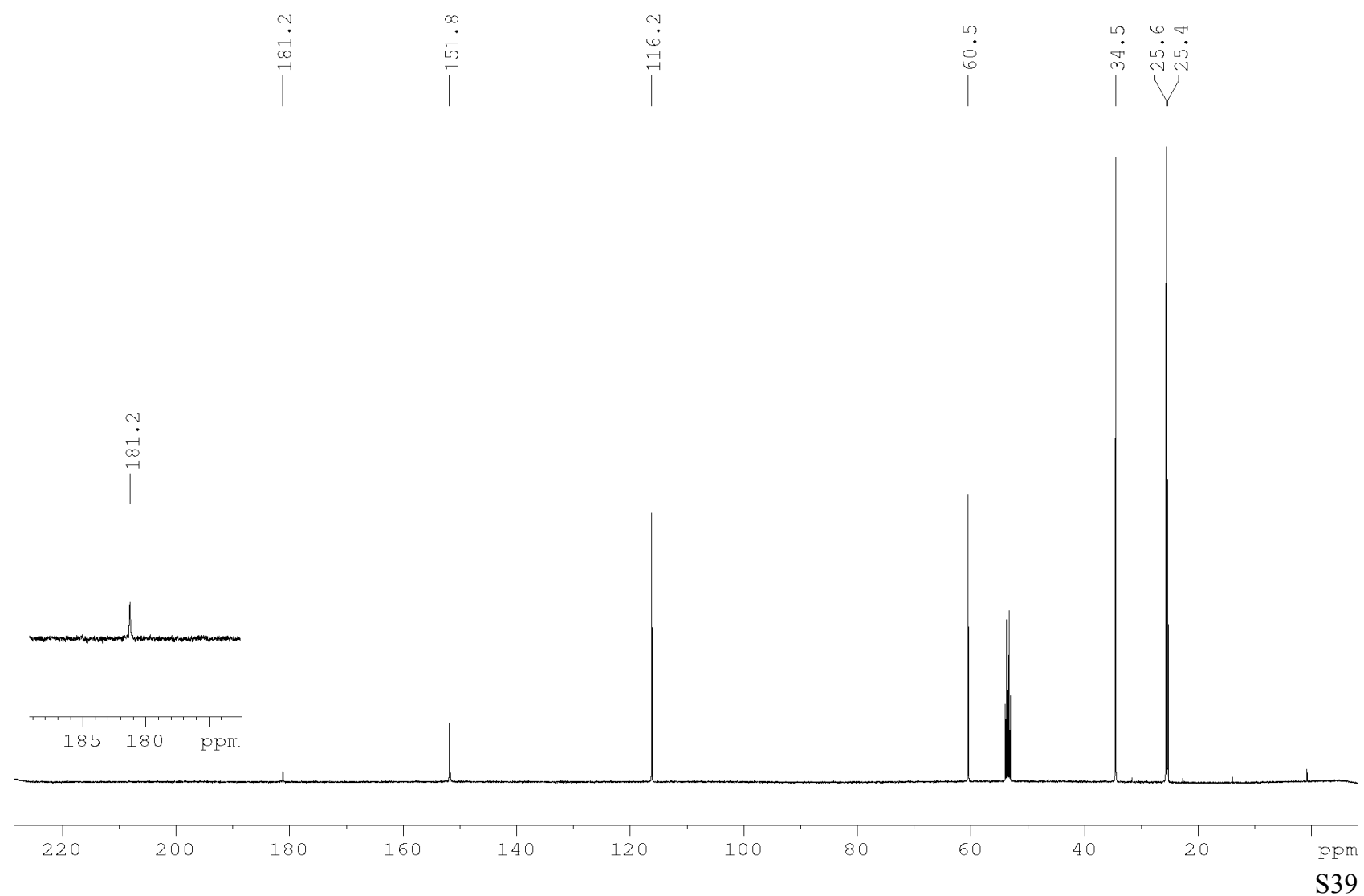


NMR spectra of the synthesised alcohols 5a-5s

${ }^{1} \mathrm{H}$ NMR (400 MHz, $\mathrm{CDCl}_{3}, 298 \mathrm{~K}$ ) of dicyclohexylmethanol, $5 \mathbf{a}$<smiles>OC(C1CCCCC1)C1CCCCC1</smiles>
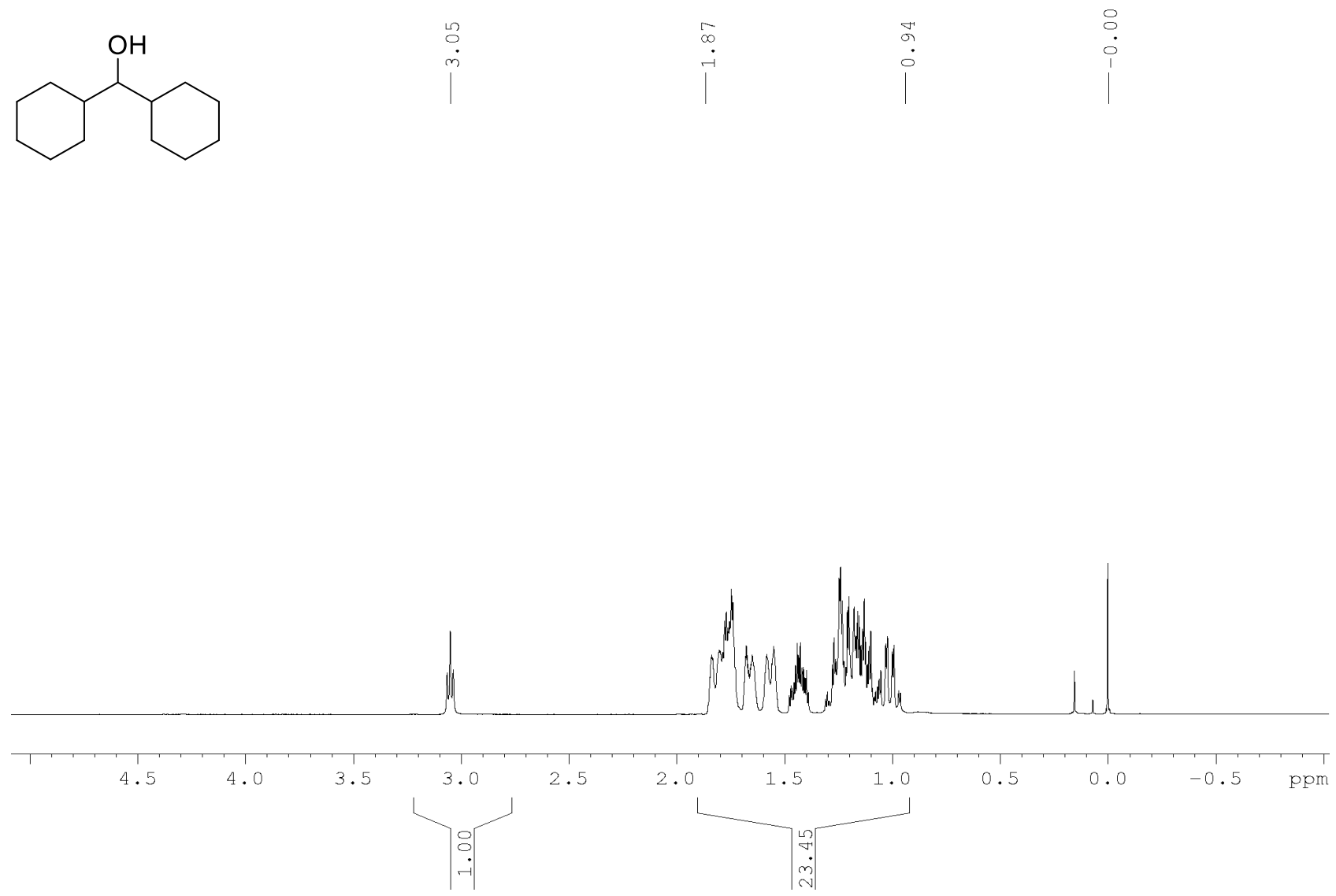

${ }^{13} \mathrm{C}-\left\{{ }^{1} \mathrm{H}\right\}$ NMR (100 MHz, $\mathrm{CDCl}$, $\left.298 \mathrm{~K}\right)$ of dicyclohexylmethanol, 5a
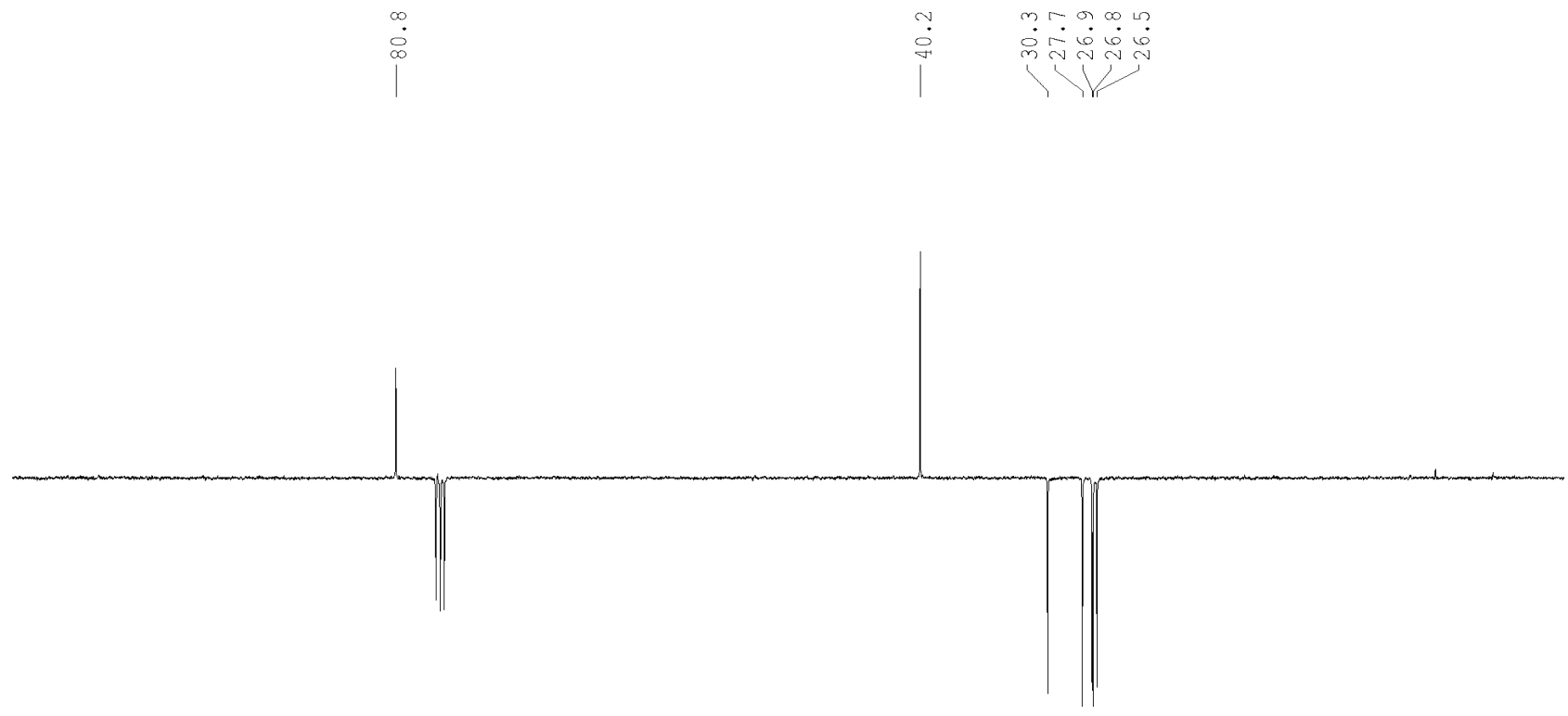

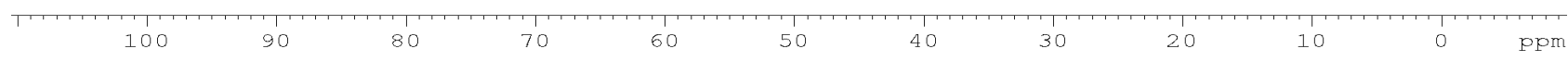


${ }^{1} \mathrm{H}$ NMR (500 MHz, $\left.\mathrm{CDCl}_{3}, 298 \mathrm{~K}\right)$ of 1-phenylethanol, $\mathbf{5 b}$

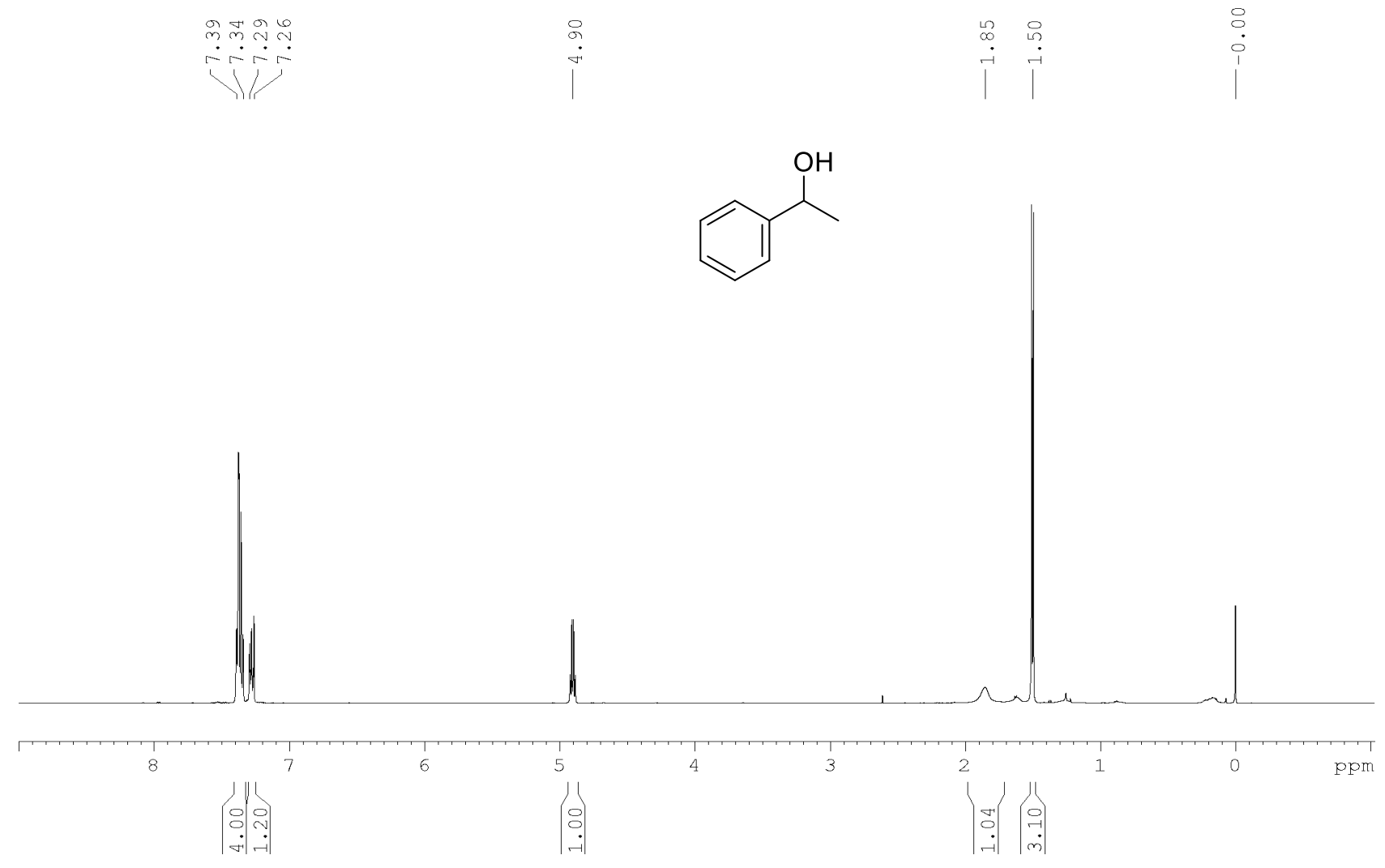

${ }^{13} \mathrm{C}-\left\{{ }^{1} \mathrm{H}\right\}$ NMR (126 MHz, $\left.\mathrm{CDCl}_{3}, 298 \mathrm{~K}\right)$ of 1-phenylethanol, 5b
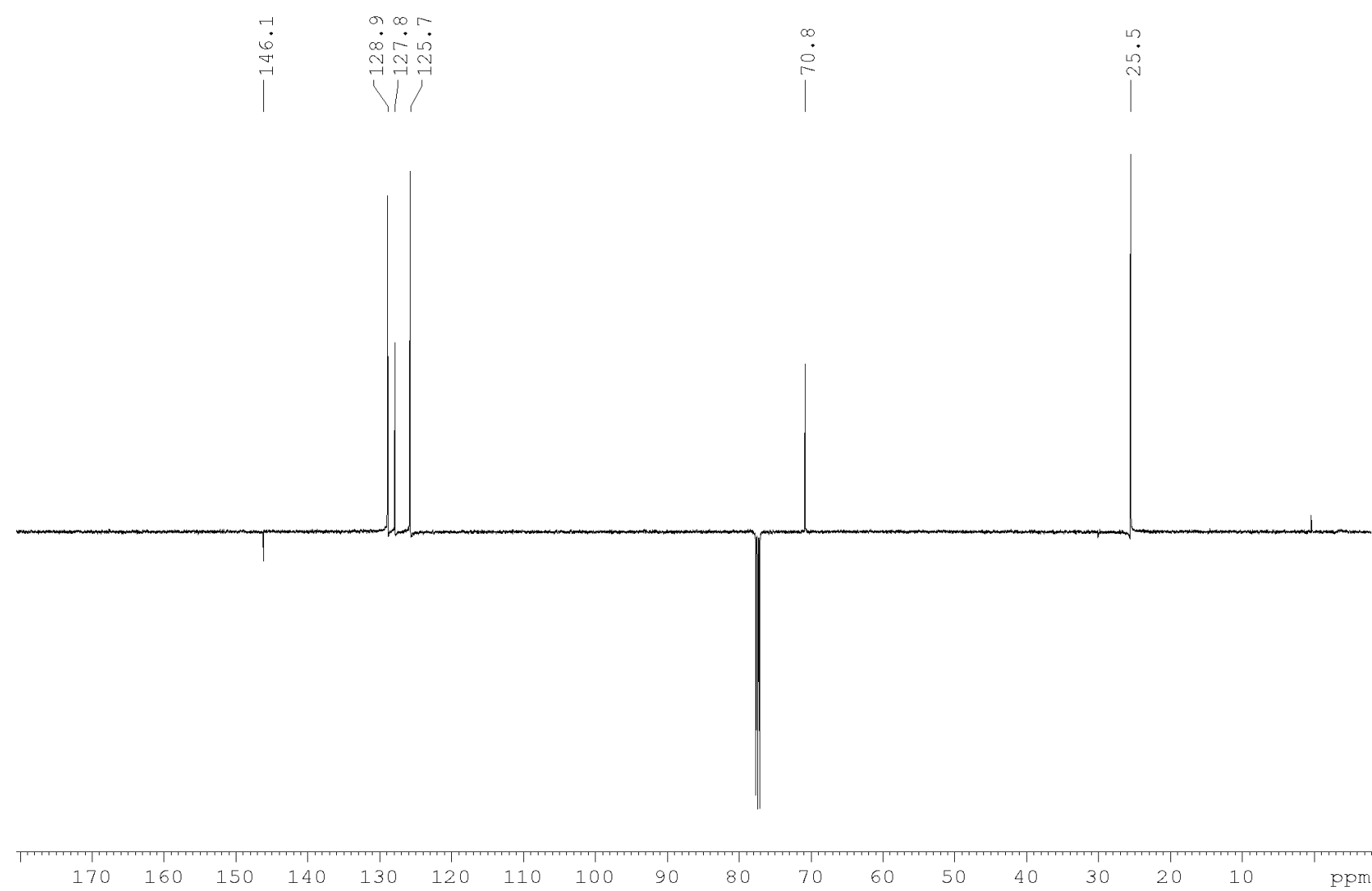
${ }^{1} \mathrm{H}$ NMR (400 MHz, $\left.\mathrm{CDCl}_{3}, 298 \mathrm{~K}\right)$ of 1-(4-nitrophenyl)ethan-1-ol, 5c

$\bigcup_{\substack{m \\ \infty}}^{\substack{m \\ \infty}}$

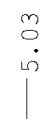
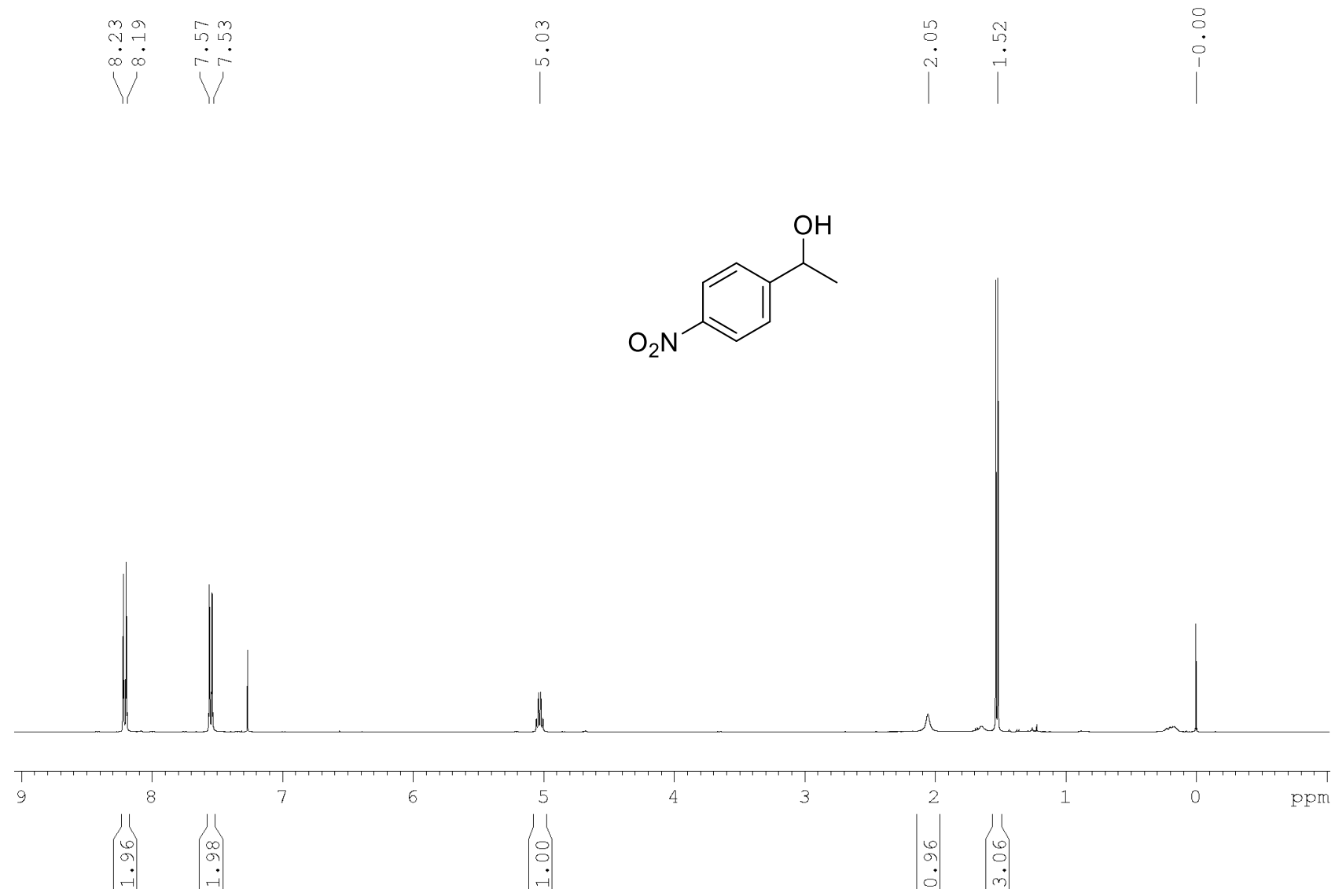

${ }^{13} \mathrm{C}-\left\{{ }^{1} \mathrm{H}\right\}$ NMR (100 MHz, $\left.\mathrm{CDCl}_{3}, 298 \mathrm{~K}\right)$ of 1-(4-nitrophenyl)ethan-1-ol, $\mathbf{5 c}$
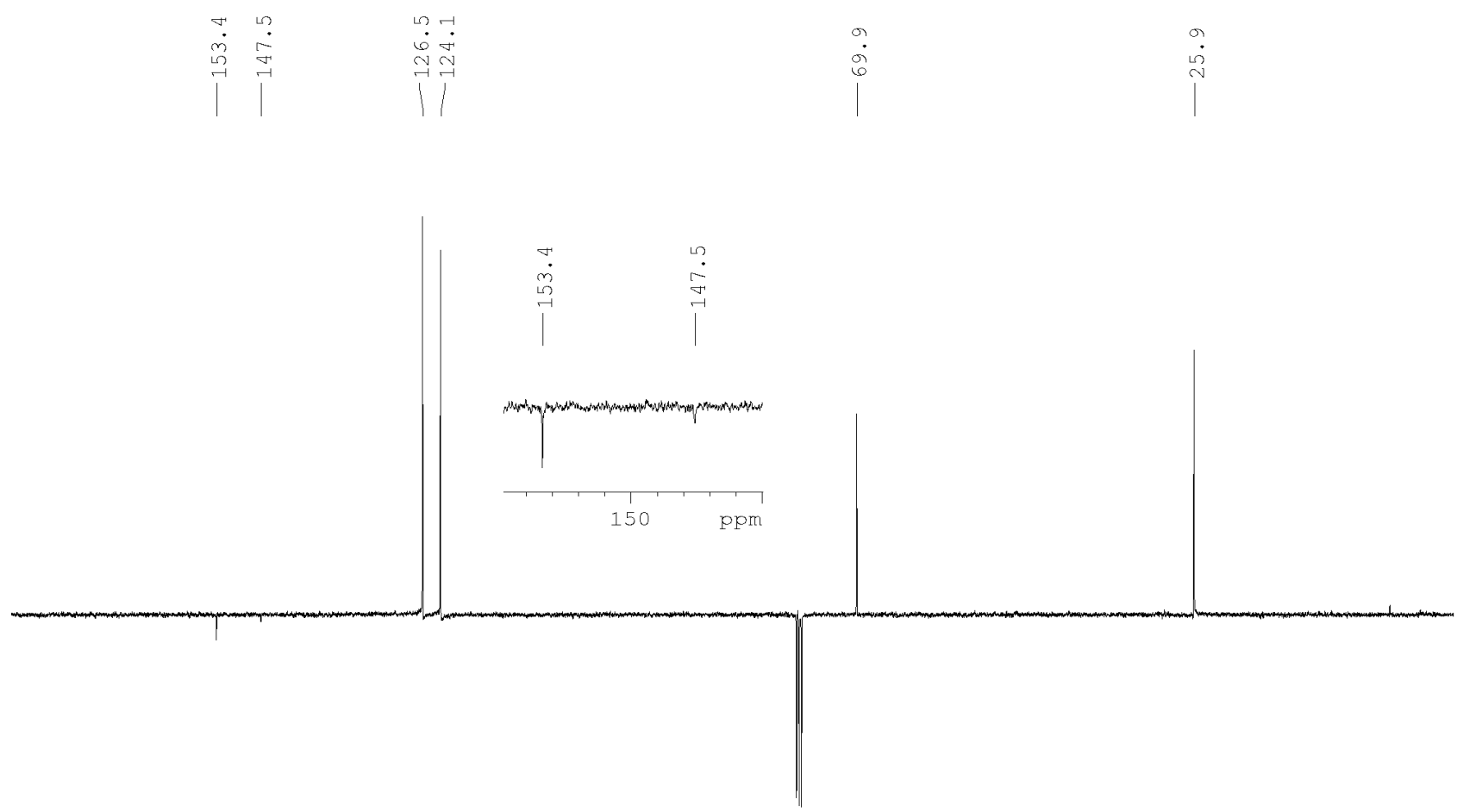

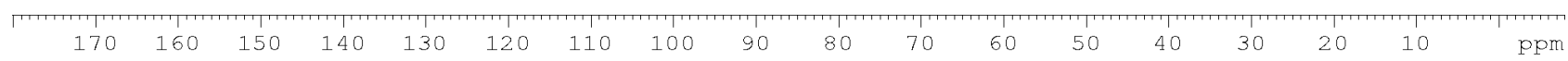


${ }^{1} \mathrm{H}$ NMR $\left(500 \mathrm{MHz}, \mathrm{CDCl}_{3}, 298 \mathrm{~K}\right)$ of 4-(1-hydroxyethyl)benzonitrile, $\mathbf{5 d}$

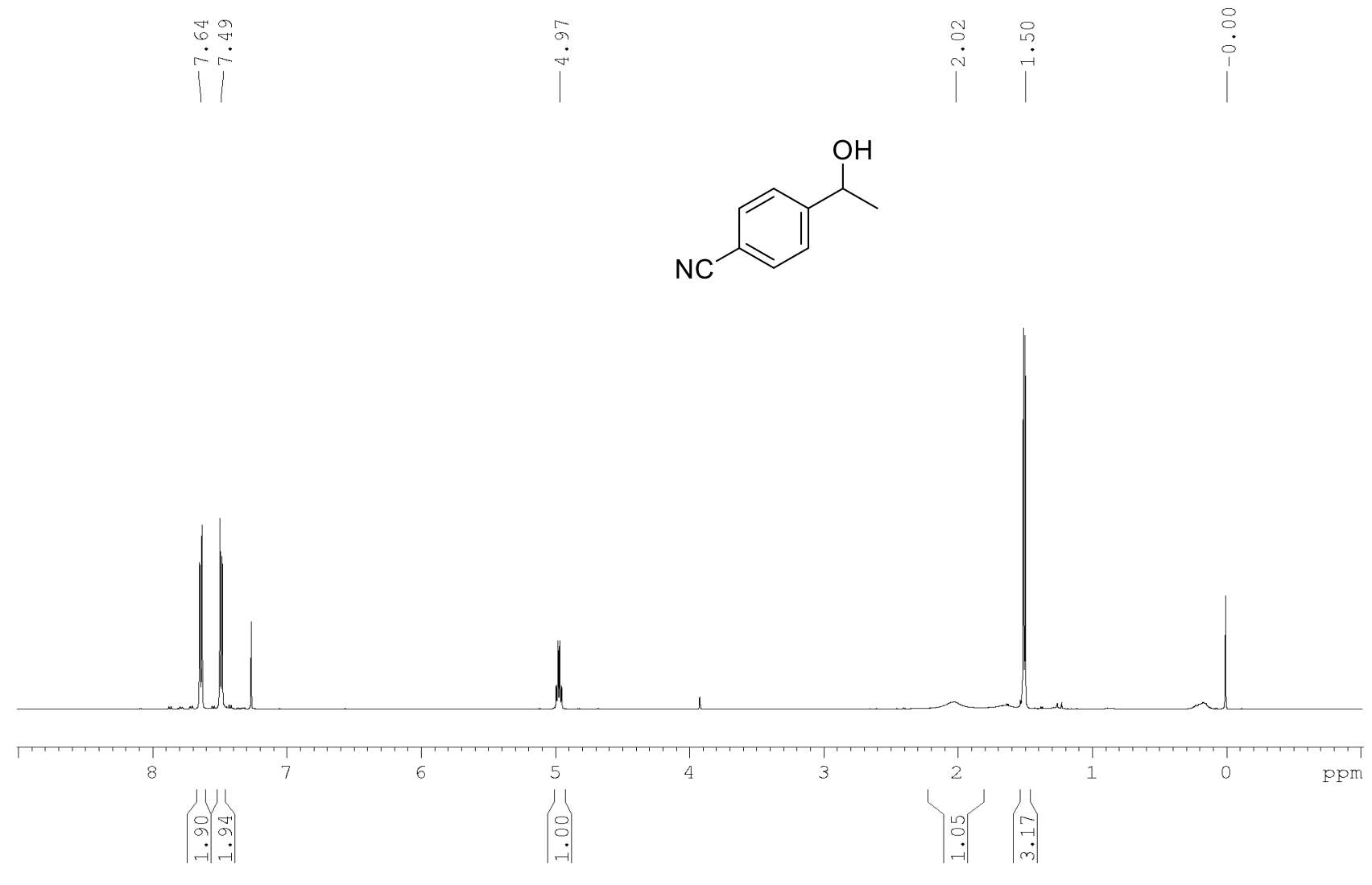

${ }^{13} \mathrm{C}-\left\{{ }^{1} \mathrm{H}\right\}$ NMR (126 MHz, $\left.\mathrm{CDCl}_{3}, 298 \mathrm{~K}\right)$ of 4-(1-hydroxyethyl)benzonitrile, 5d
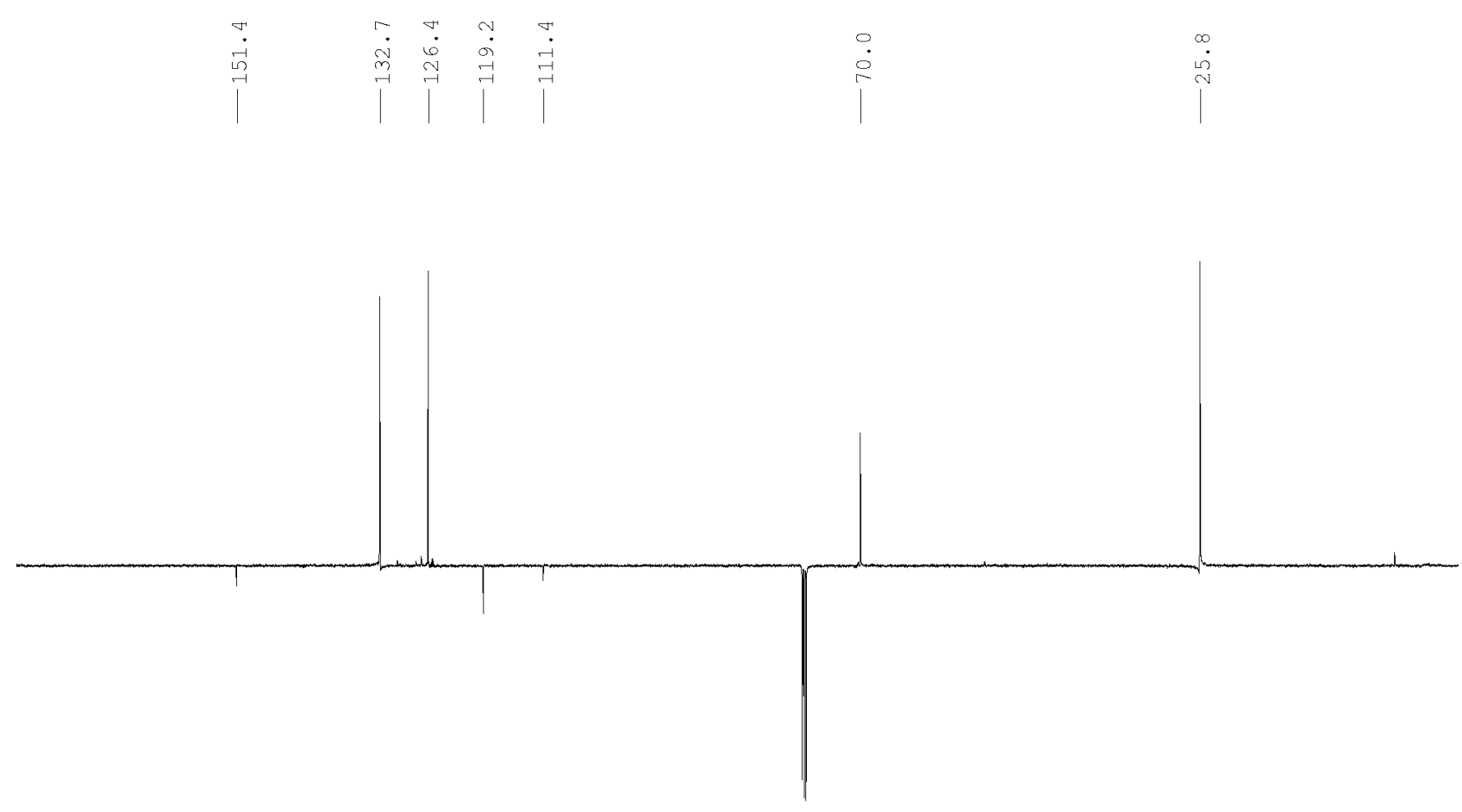

$\begin{array}{llllllllllllllllll}170 & 160 & 150 & 140 & 130 & 120 & 110 & 100 & 90 & 80 & 70 & 60 & 50 & 40 & 30 & 20 & 10 & 0\end{array}$ 
${ }^{1} \mathrm{H}$ NMR (400 MHz, $\left.\mathrm{CDCl}_{3}, 298 \mathrm{~K}\right)$ of 1-(4-chlorophenyl)ethan-1-ol, 5e

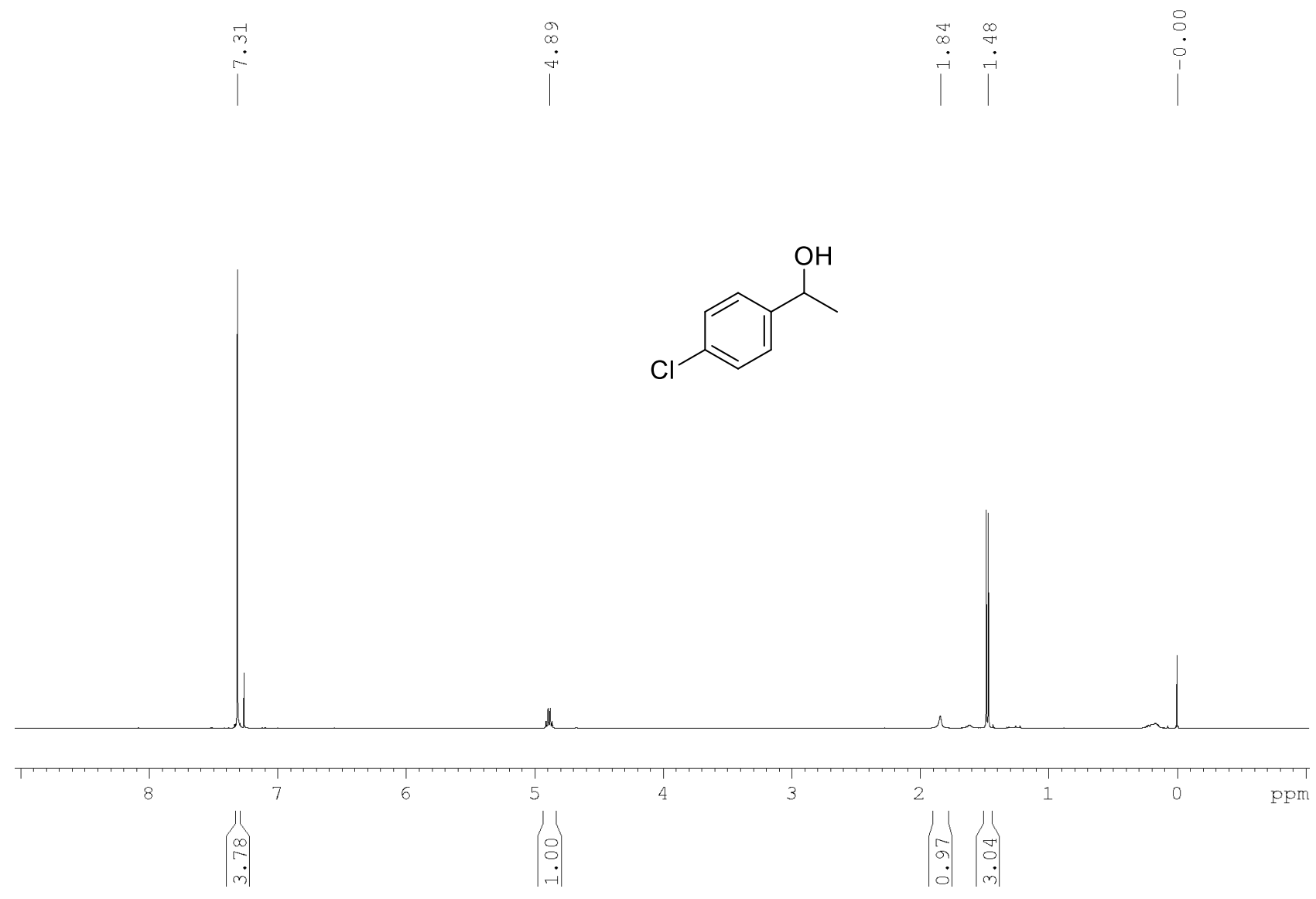

${ }^{13} \mathrm{C}-\left\{{ }^{1} \mathrm{H}\right\}$ NMR $\left(100 \mathrm{MHz}, \mathrm{CDCl}_{3}, 298 \mathrm{~K}\right)$ of 1-(4-chlorophenyl)ethan-1-ol, $\mathbf{5 e}$

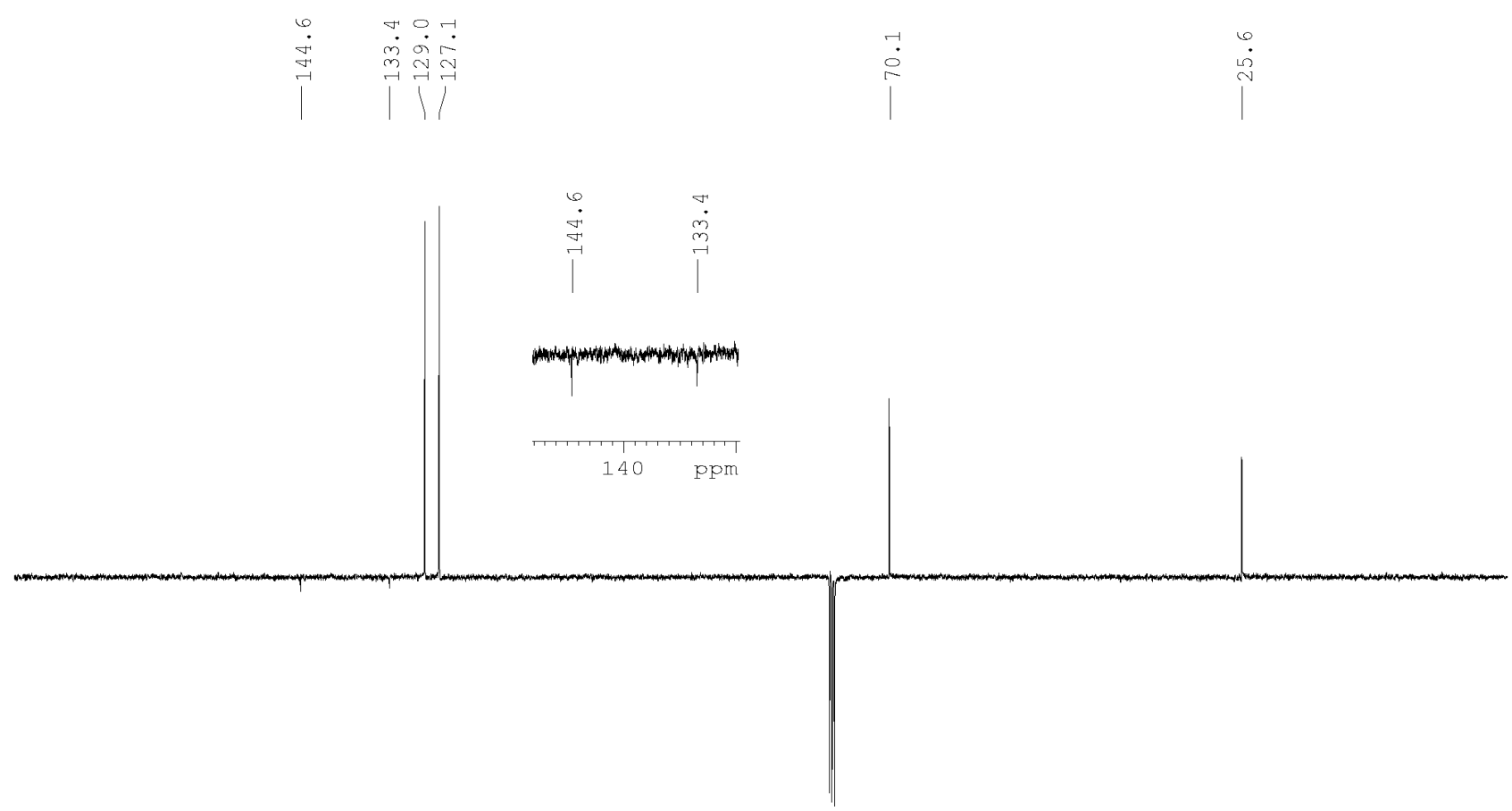

$\begin{array}{llllllllllllllllllll}170 & 160 & 150 & 140 & 130 & 120 & 110 & 100 & 90 & 80 & 70 & 60 & 50 & 40 & 30 & 20 & 10 & \text { ppm }\end{array}$ 
${ }^{1} \mathrm{H}$ NMR (500 MHz, $\left.\mathrm{CDCl}_{3}, 298 \mathrm{~K}\right)$ of 1-(4-methoxyphenyl)ethan-1-ol, $\mathbf{5 f}$
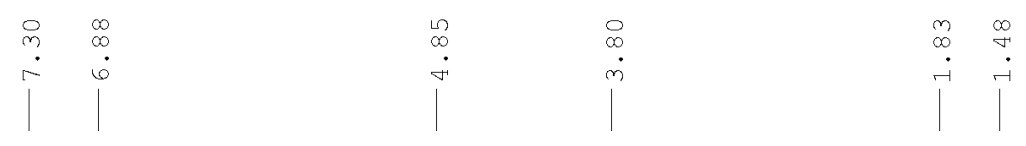

$$
\begin{aligned}
& 8 \\
& 8 \\
& 0 \\
& 1
\end{aligned}
$$

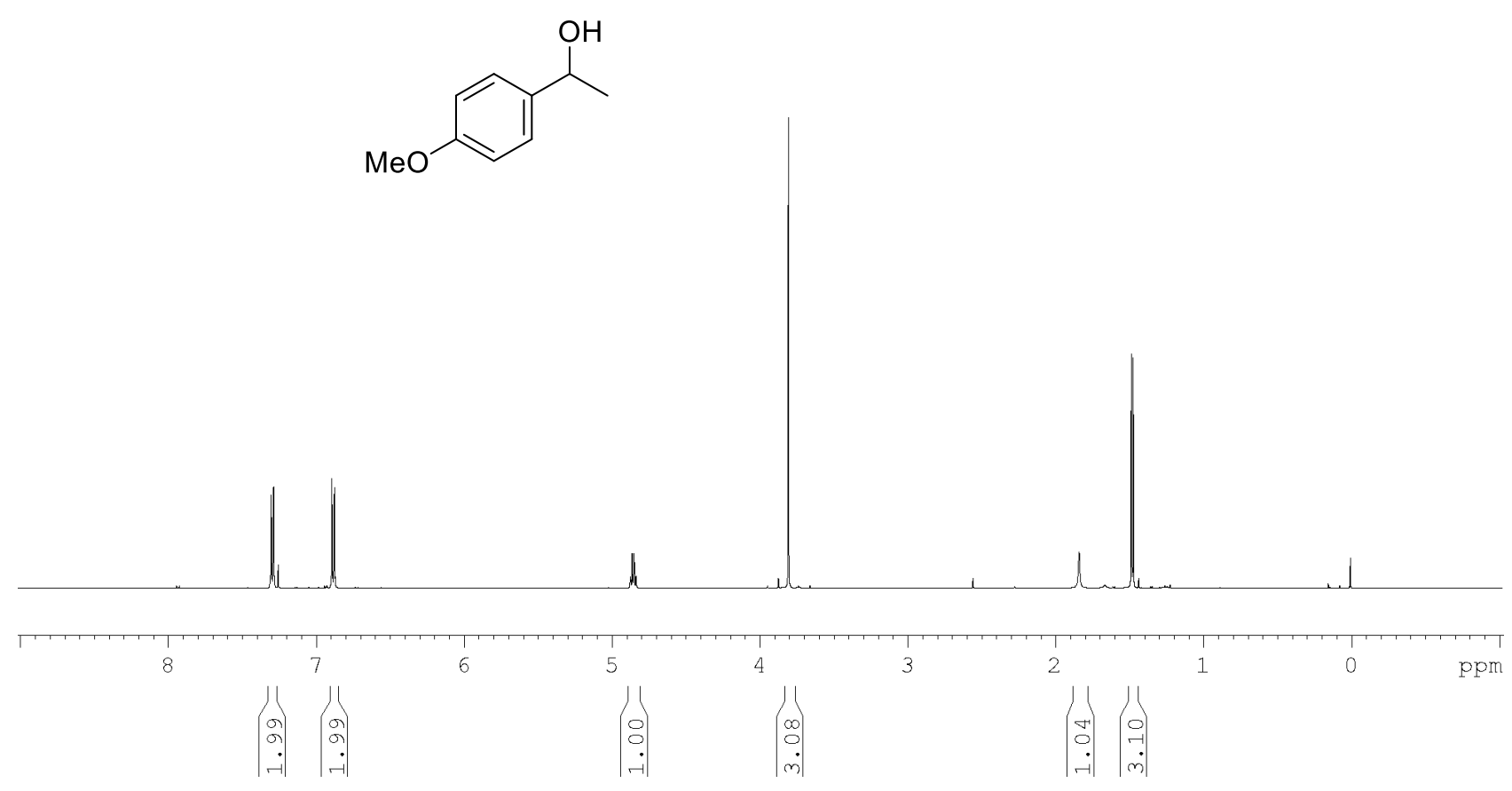

${ }^{13} \mathrm{C}-\left\{{ }^{1} \mathrm{H}\right\}$ NMR (126 MHz, $\left.\mathrm{CDCl}_{3}, 298 \mathrm{~K}\right)$ of 1-(4-methoxyphenyl)ethan-1-ol, $\mathbf{5 f}$

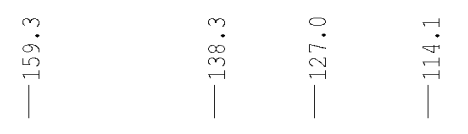

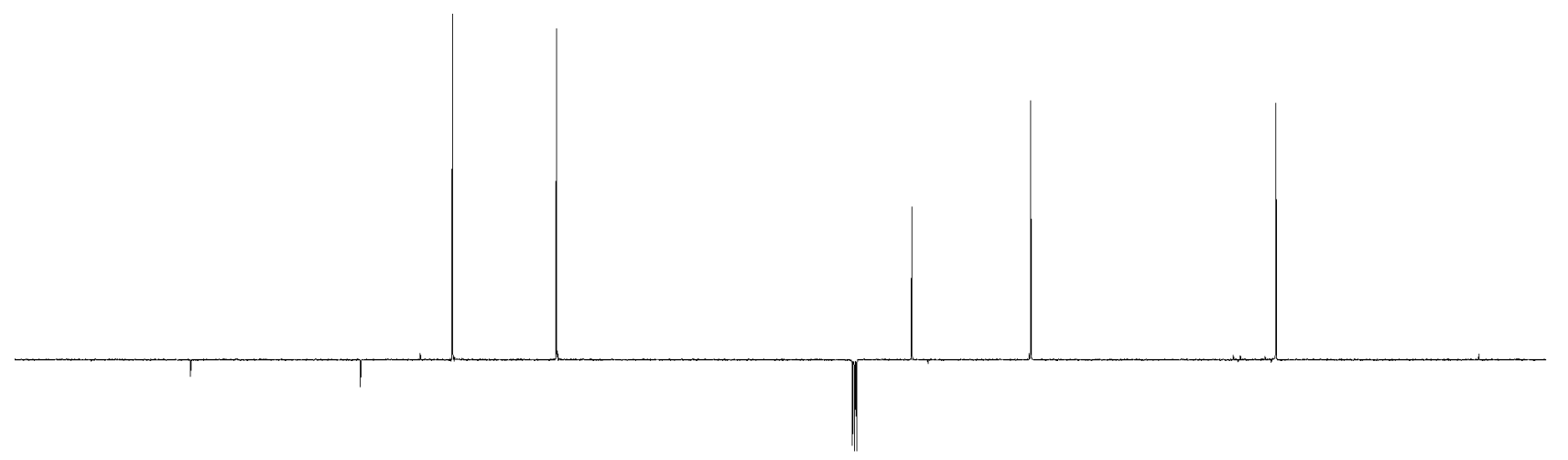


${ }^{1} \mathrm{H}$ NMR $\left(500 \mathrm{MHz}, \mathrm{CDCl}_{3}, 298 \mathrm{~K}\right)$ of 1-(o-tolyl)ethan-1-ol, $\mathbf{5 g}$

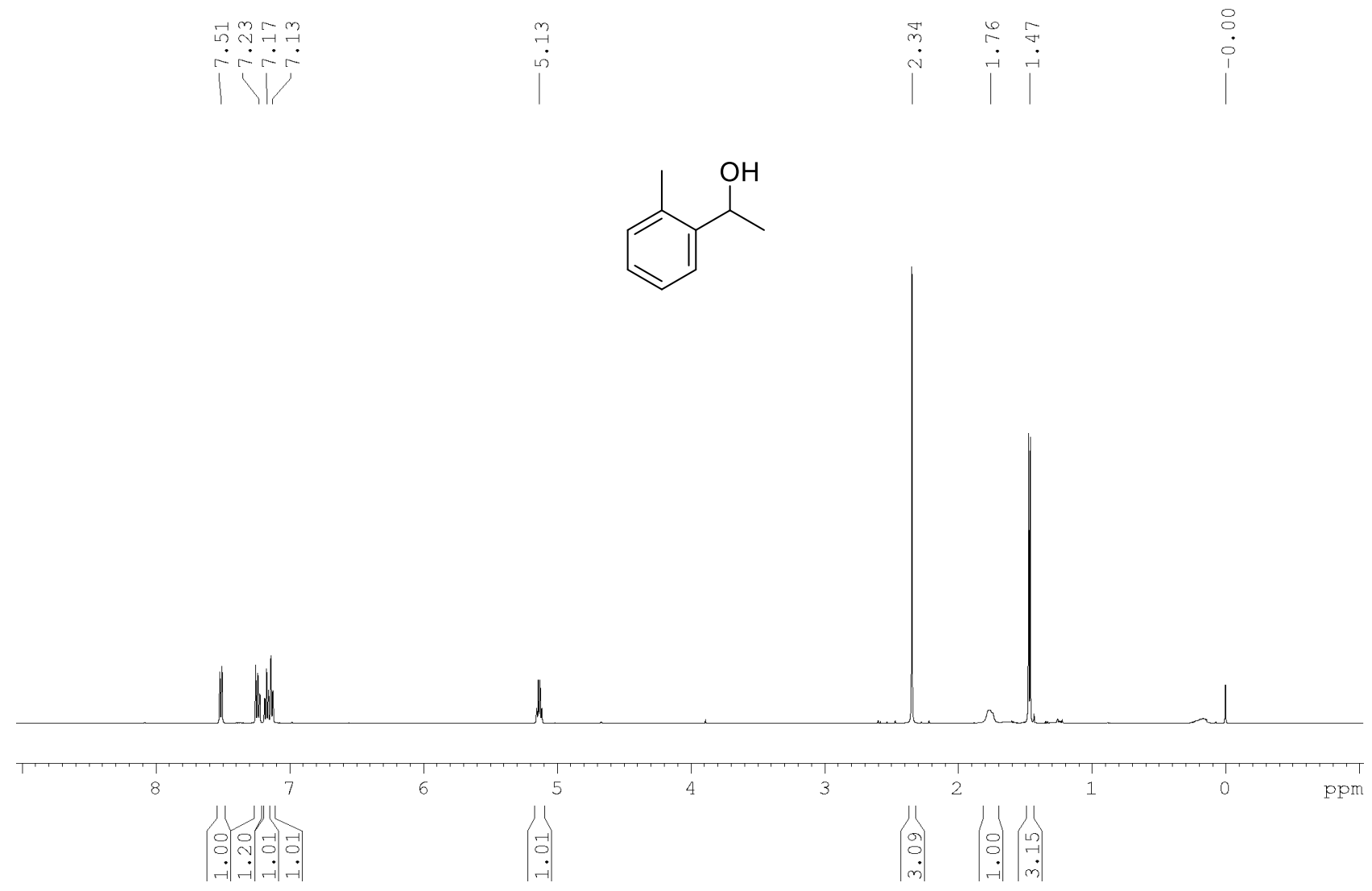

${ }^{13} \mathrm{C}-\left\{{ }^{1} \mathrm{H}\right\}$ NMR $\left(126 \mathrm{MHz}, \mathrm{CDCl}_{3}, 298 \mathrm{~K}\right)$ of 1-(o-tolyl)ethan-1-ol, $\mathbf{5 g}$

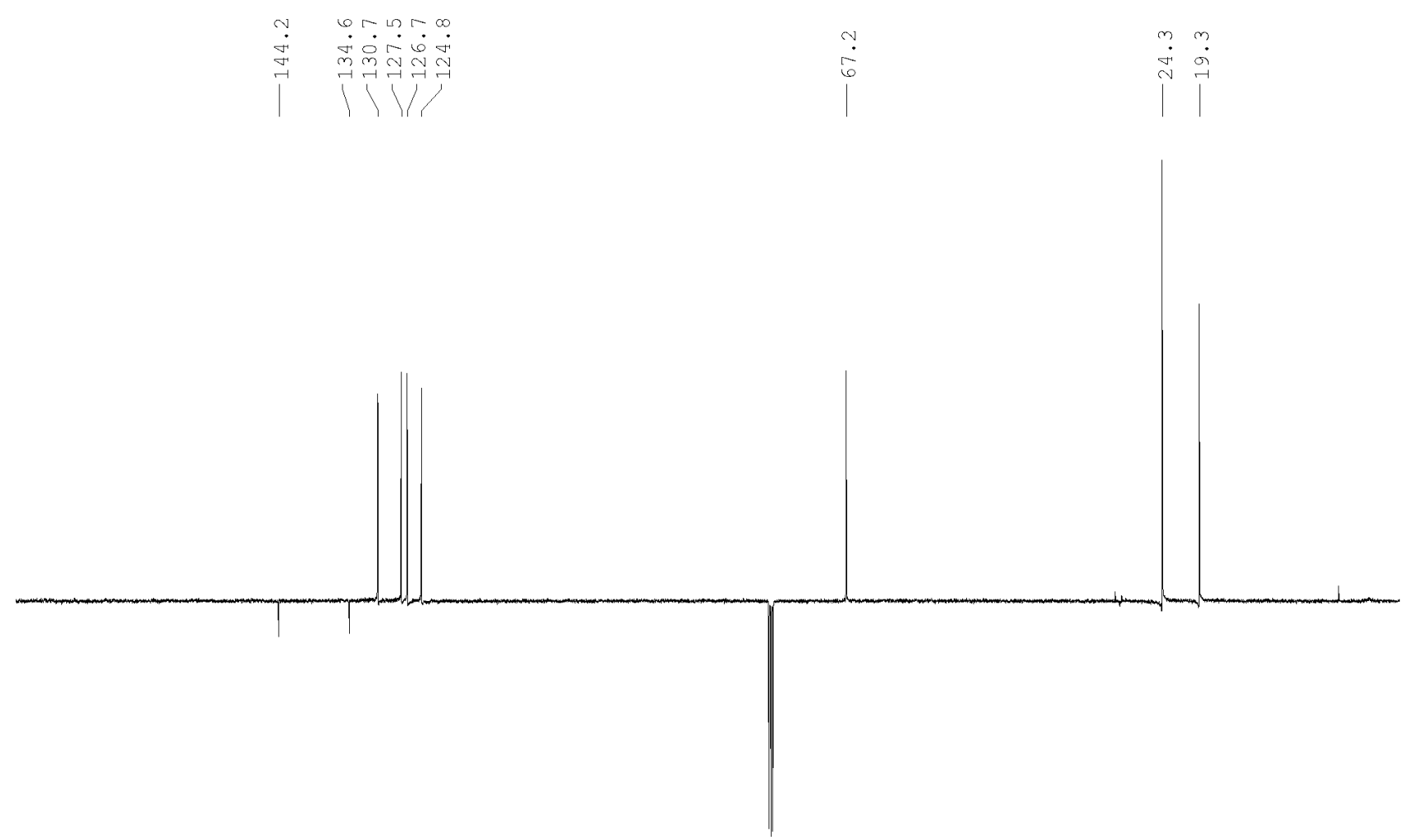

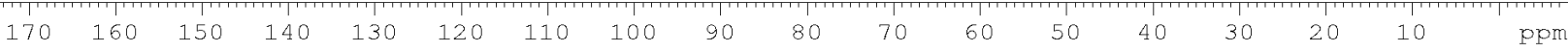


${ }^{1} \mathrm{H}$ NMR (500 MHz, $\left.\mathrm{CDCl}_{3}, 298 \mathrm{~K}\right)$ of 1-(6-methoxynaphthalen-2-yl)ethan-1-ol, $\mathbf{5 h}$

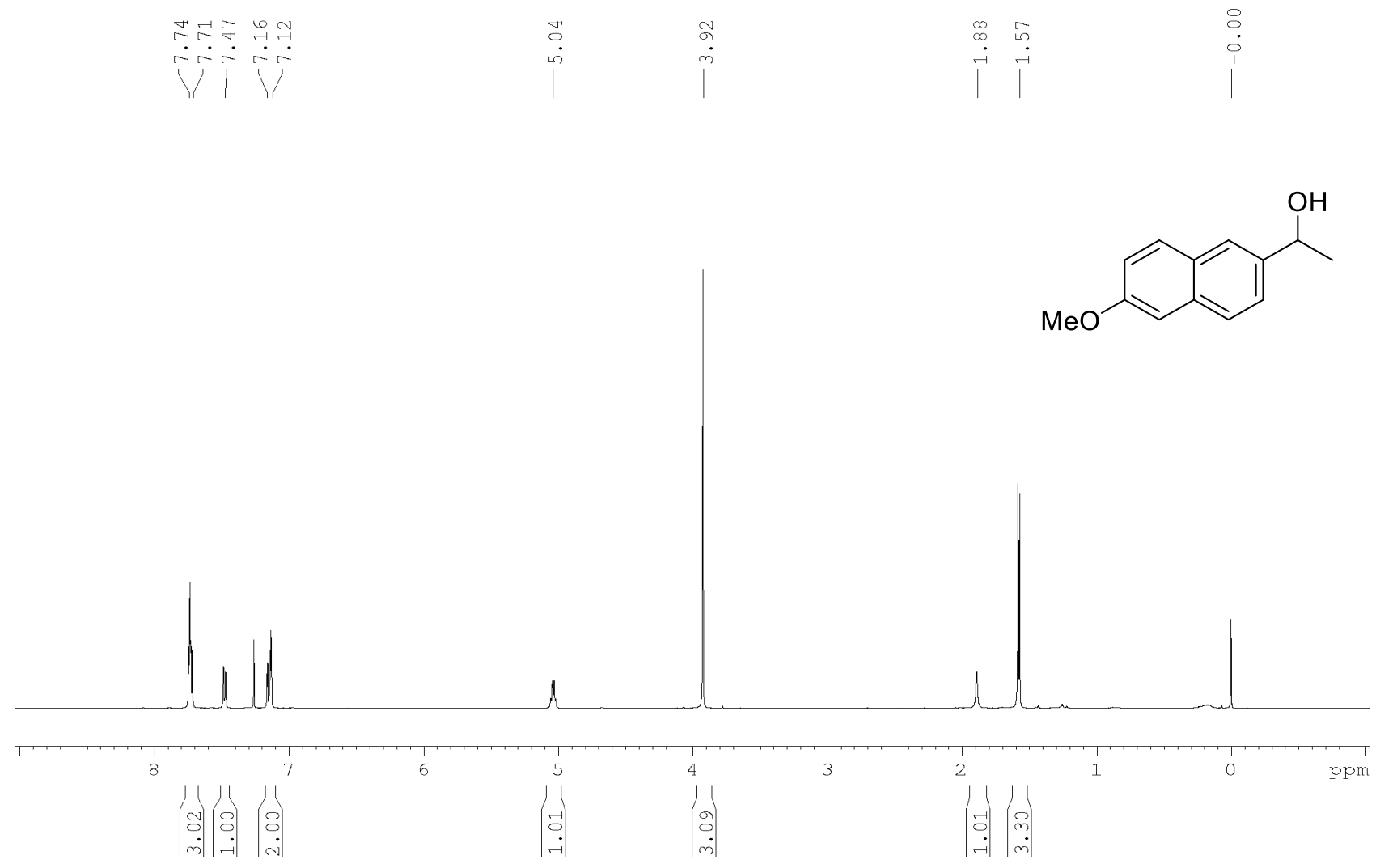

${ }^{13} \mathrm{C}-\left\{{ }^{1} \mathrm{H}\right\}$ NMR (126 MHz, $\left.\mathrm{CDCl}_{3}, 298 \mathrm{~K}\right)$ of 1-(6-methoxynaphthalen-2-yl)ethan-1-ol, $\mathbf{5 h}$
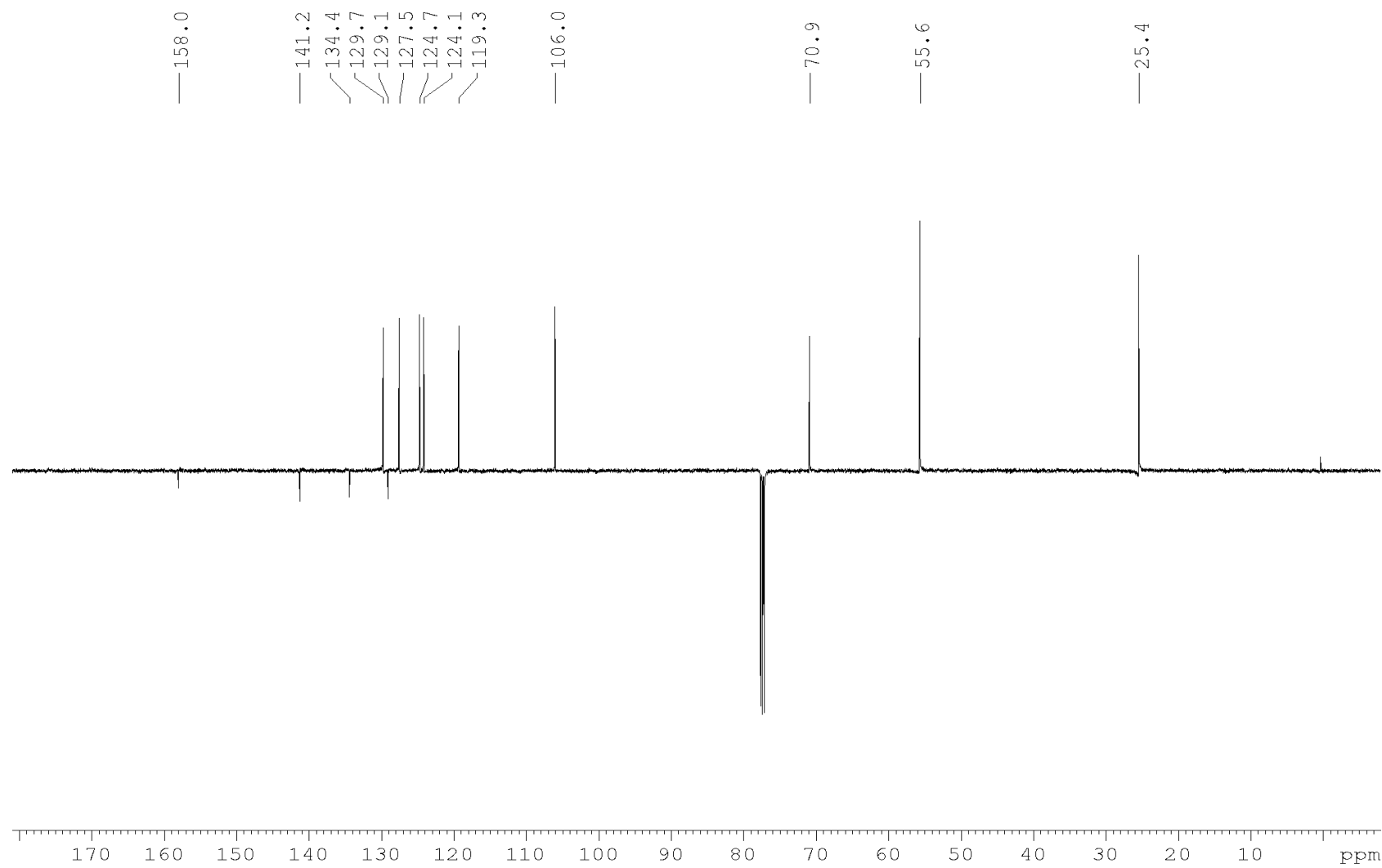
${ }^{1} \mathrm{H}$ NMR (500 MHz, CDCl 3 , 298K) of 2,2-dimethyl-1-phenylpropan-1-ol, $\mathbf{5 i}$

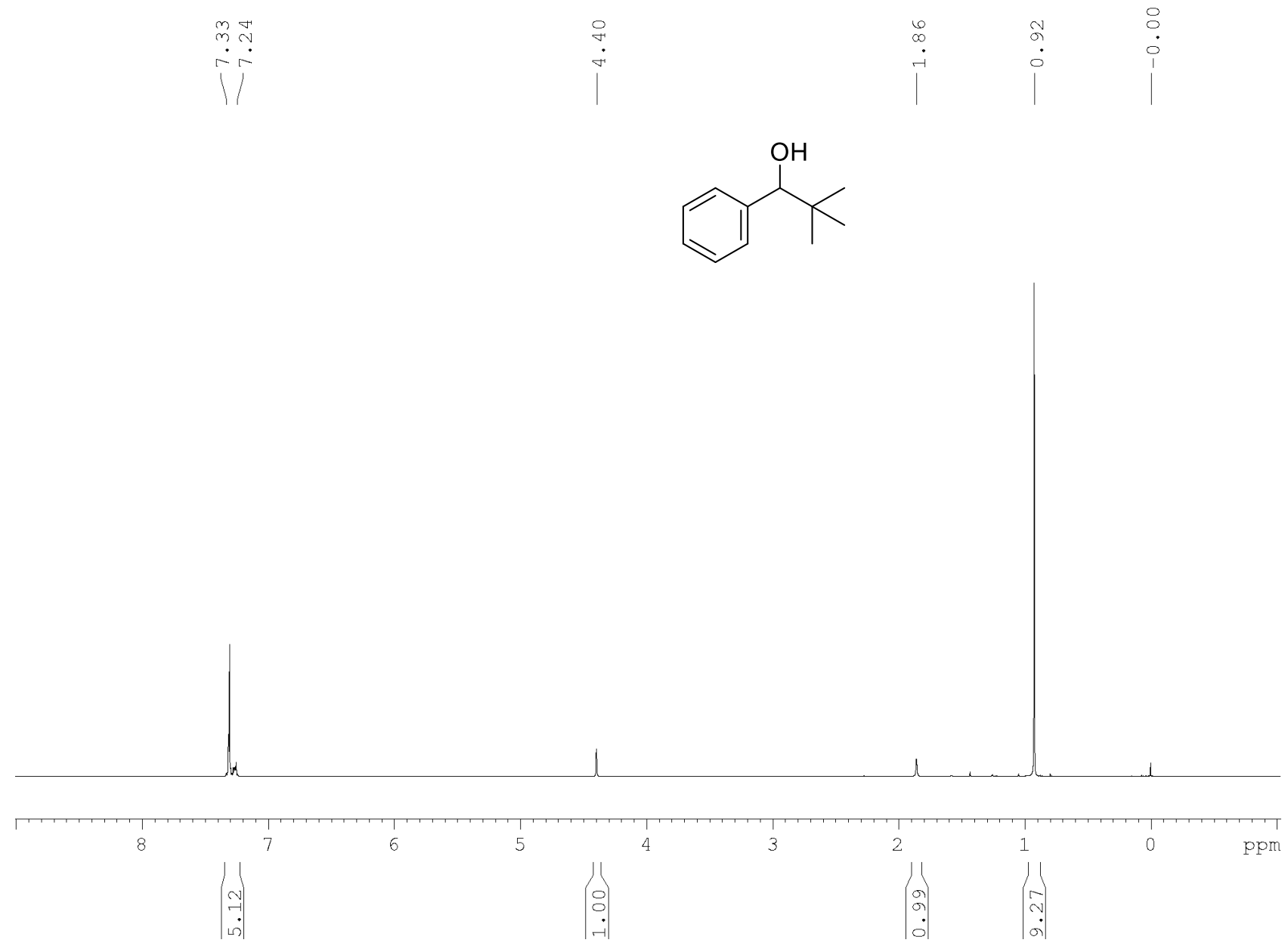

${ }^{13} \mathrm{C}-\left\{{ }^{1} \mathrm{H}\right\}$ NMR (126 MHz, $\left.\mathrm{CDCl}_{3}, 298 \mathrm{~K}\right)$ of 2,2-dimethyl-1-phenylpropan-1-ol, 5i

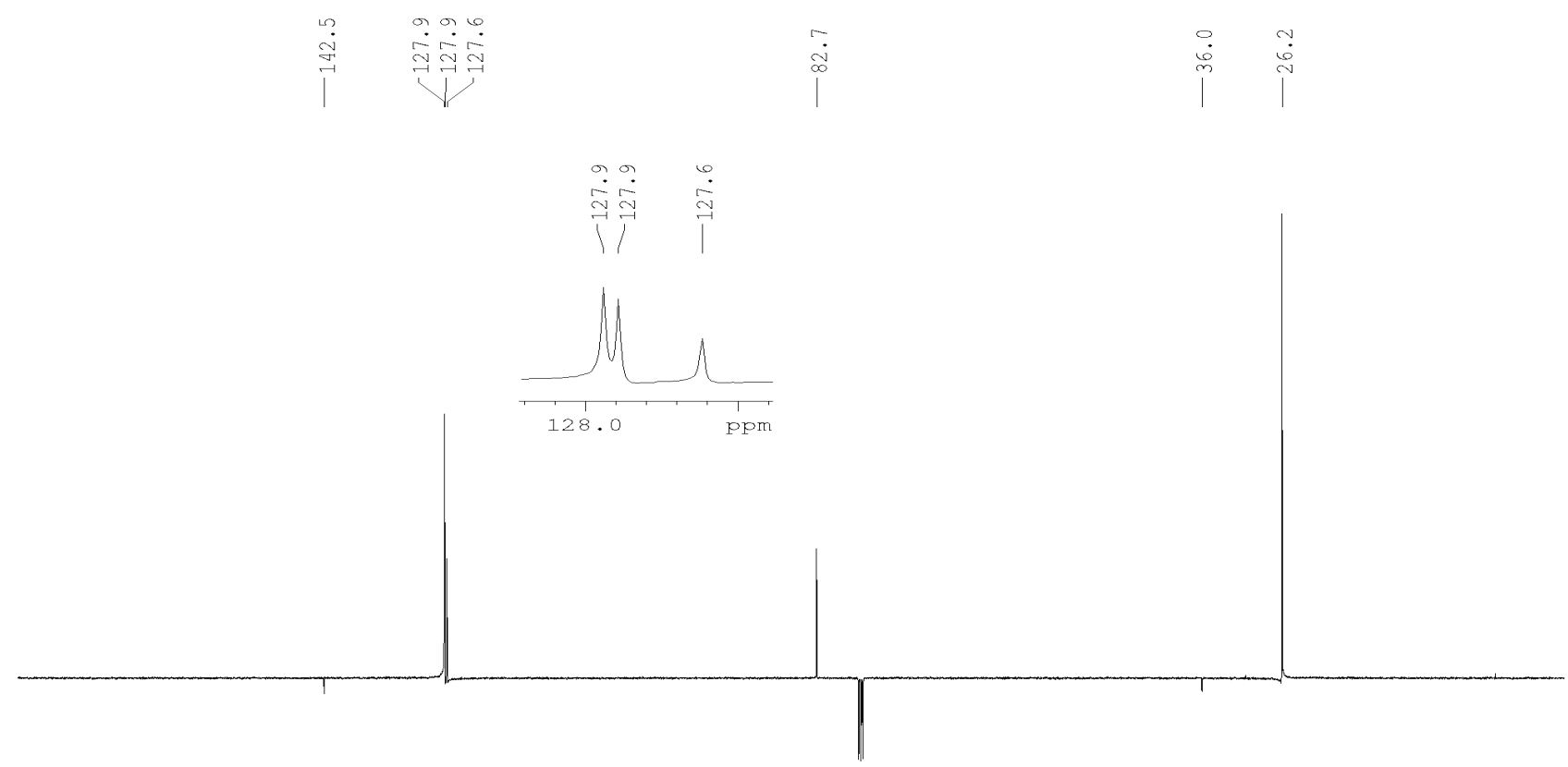

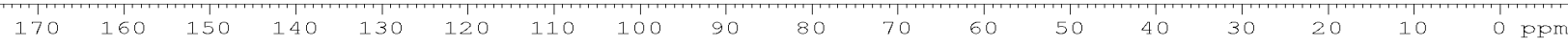


${ }^{1} \mathrm{H}$ NMR (500 MHz, $\left.\mathrm{CDCl}_{3}, 298 \mathrm{~K}\right)$ of diphenylmethanol, $\mathbf{5 j}$

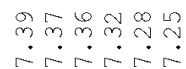

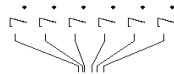

$\stackrel{\leftrightarrow}{\infty}$
$i$
$\stackrel{\leftrightarrow}{0}$<smiles>OC(c1ccccc1)c1ccccc1</smiles>

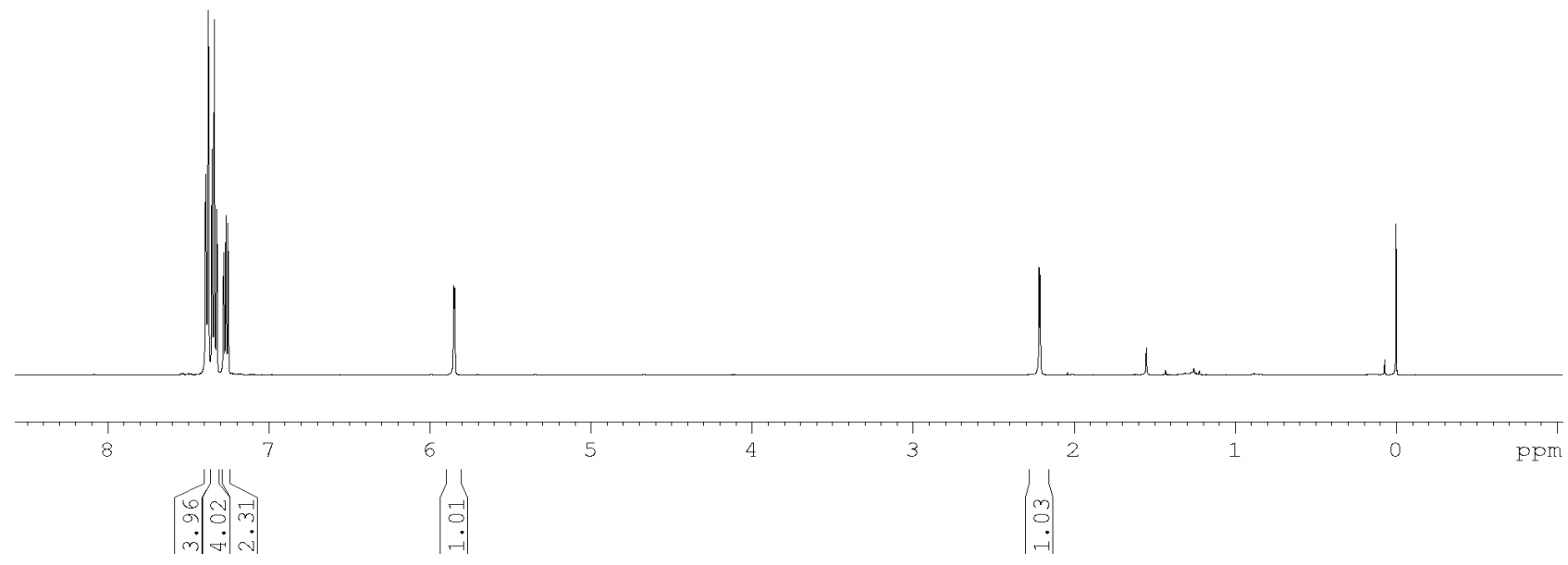

${ }^{13} \mathrm{C}-\left\{{ }^{1} \mathrm{H}\right\}$ NMR (126 MHz, $\left.\mathrm{CDCl}_{3}, 298 \mathrm{~K}\right)$ of diphenylmethanol, $\mathbf{5} \mathbf{j}$
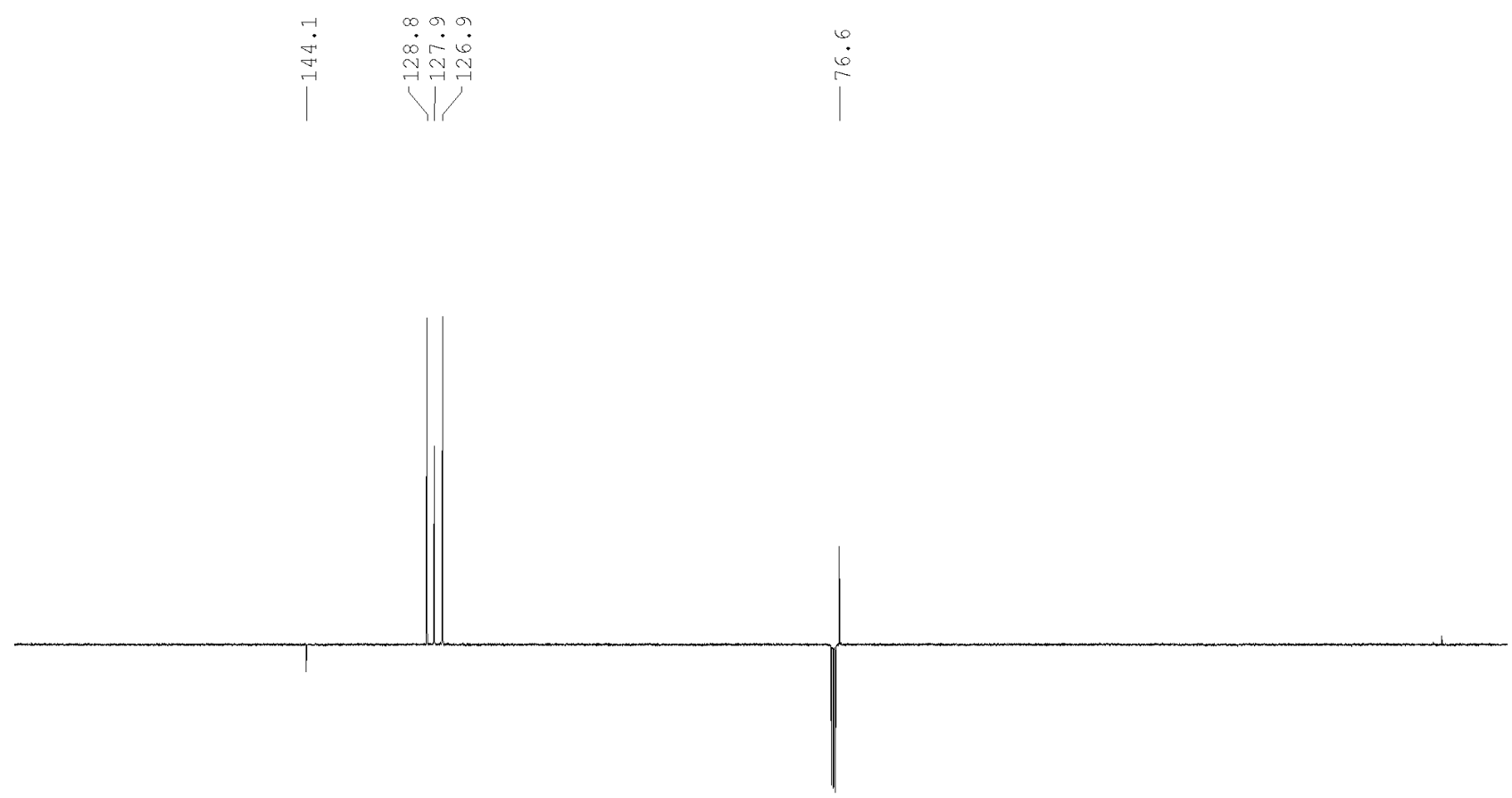
${ }^{1} \mathrm{H}$ NMR (500 MHz, $\left.\mathrm{CDCl}_{3}, 298 \mathrm{~K}\right)$ of bis(4-methoxyphenyl)methanol, 5k

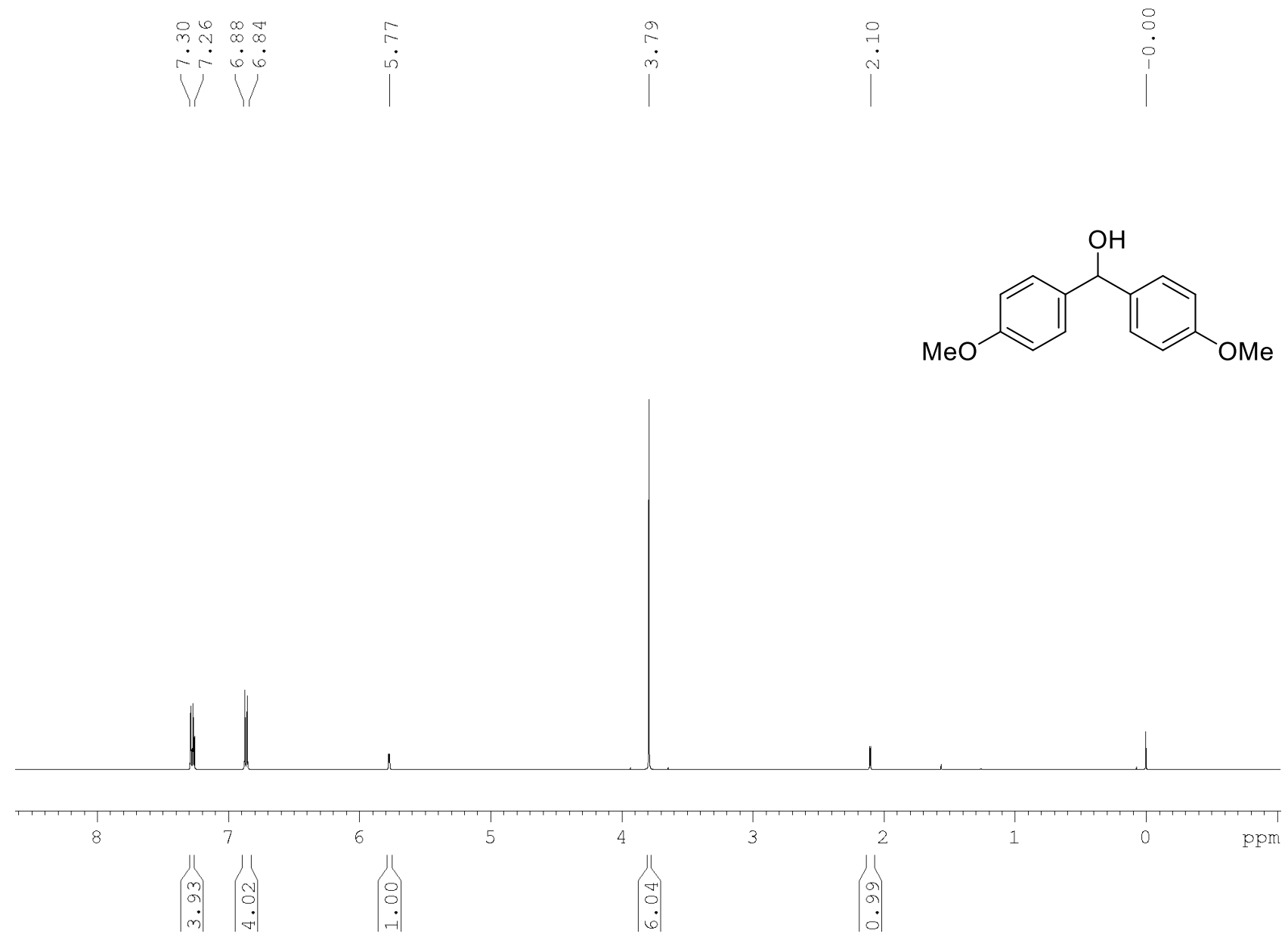

${ }^{13} \mathrm{C}-\left\{{ }^{1} \mathrm{H}\right\}$ NMR $\left(126 \mathrm{MHz}, \mathrm{CDCl}_{3}, 298 \mathrm{~K}\right)$ of bis(4-methoxyphenyl)methanol, 5k

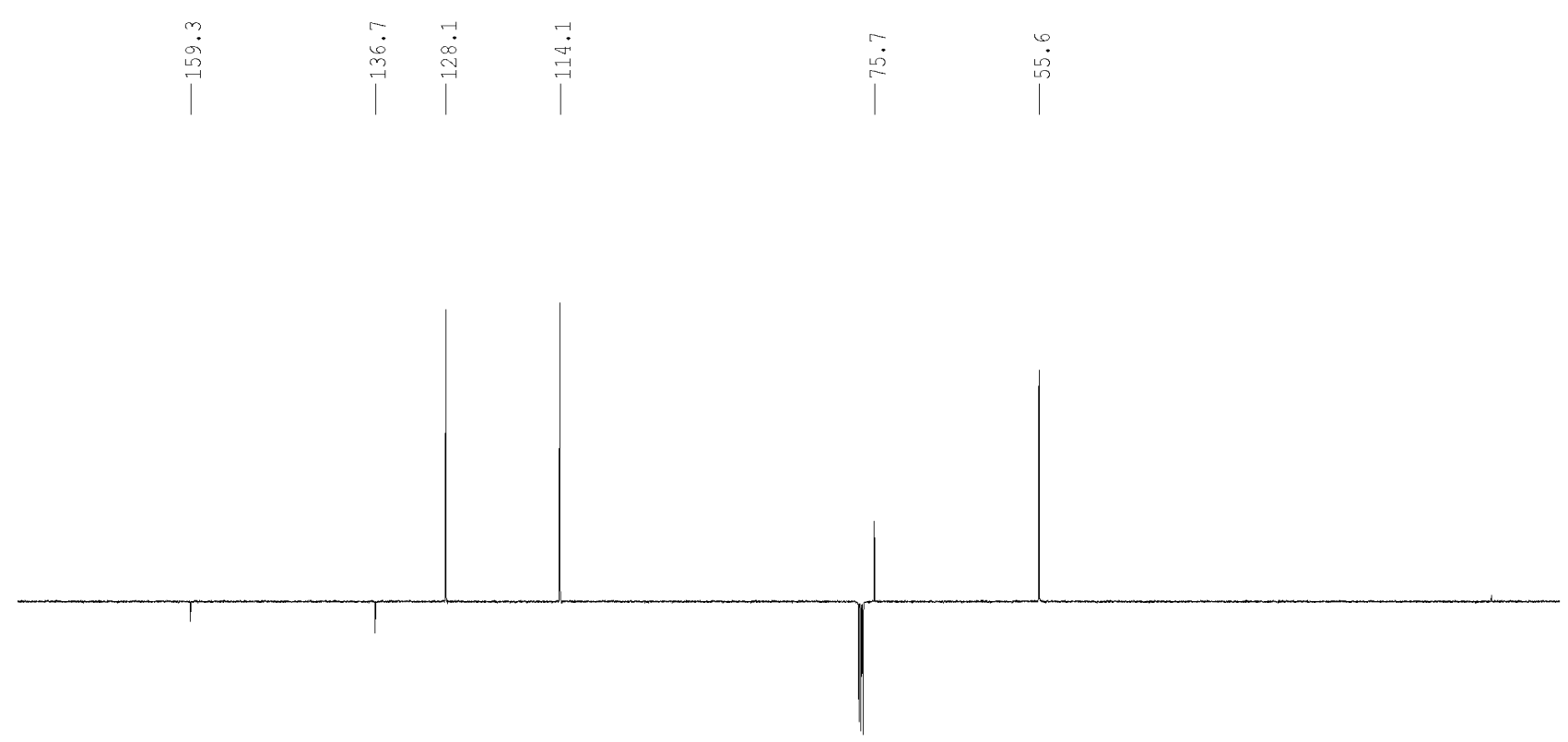

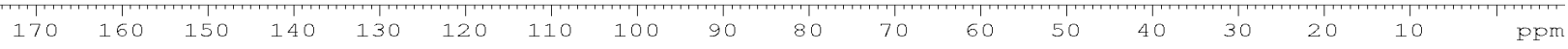


${ }^{1} \mathrm{H}$ NMR (500 MHz, $\left.\mathrm{CDCl}_{3}, 298 \mathrm{~K}\right)$ of 10,11-dihydro-5H-dibenzo[a,d][7]annulen-5-ol, 5l
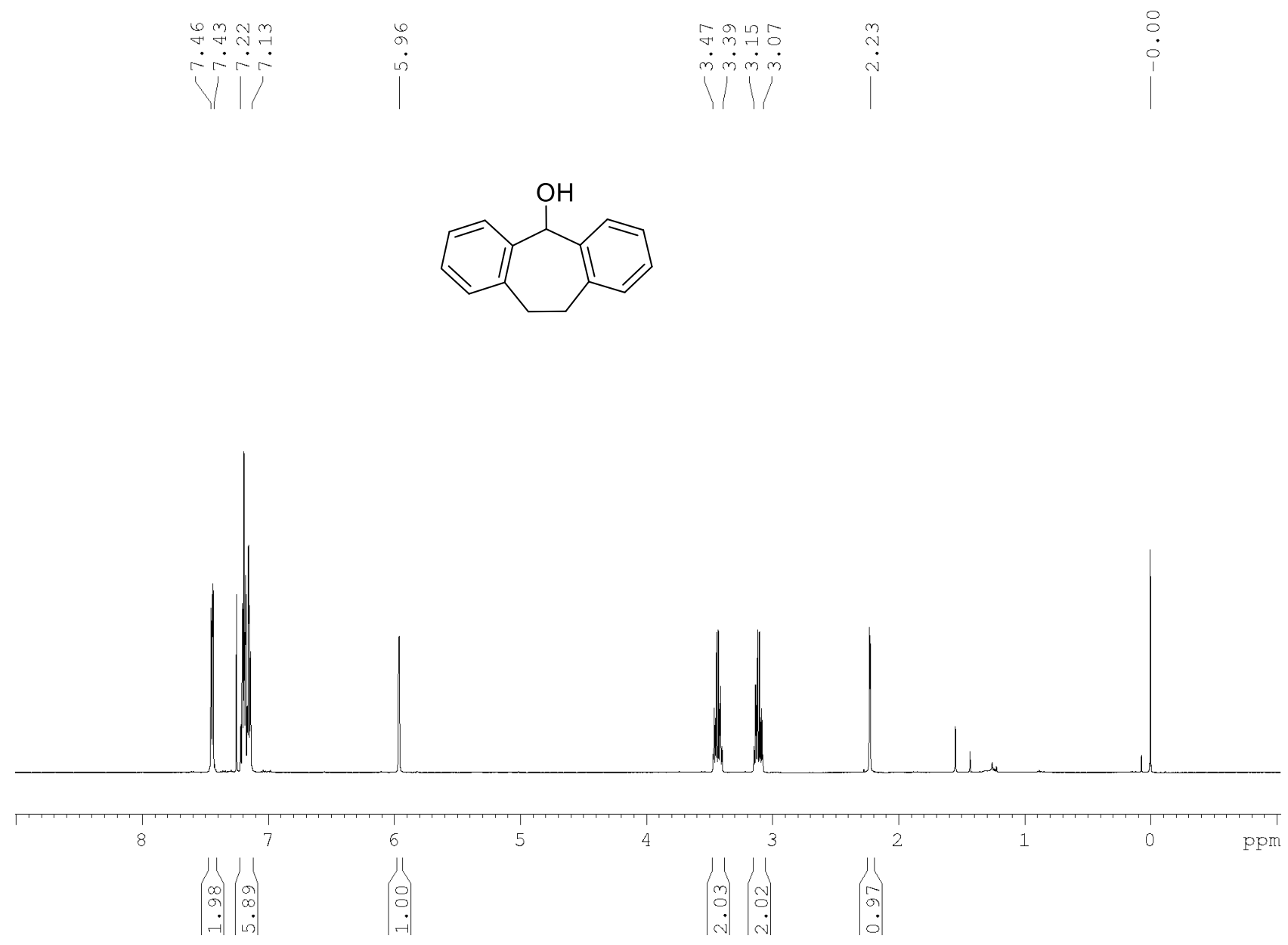

${ }^{13} \mathrm{C}-\left\{{ }^{1} \mathrm{H}\right\}$ NMR (126 MHz, $\left.\mathrm{CDCl}_{3}, 298 \mathrm{~K}\right)$ of 10,11-dihydro-5H-dibenzo[a,d][7]annulen-5-ol, $\mathbf{5 1}$
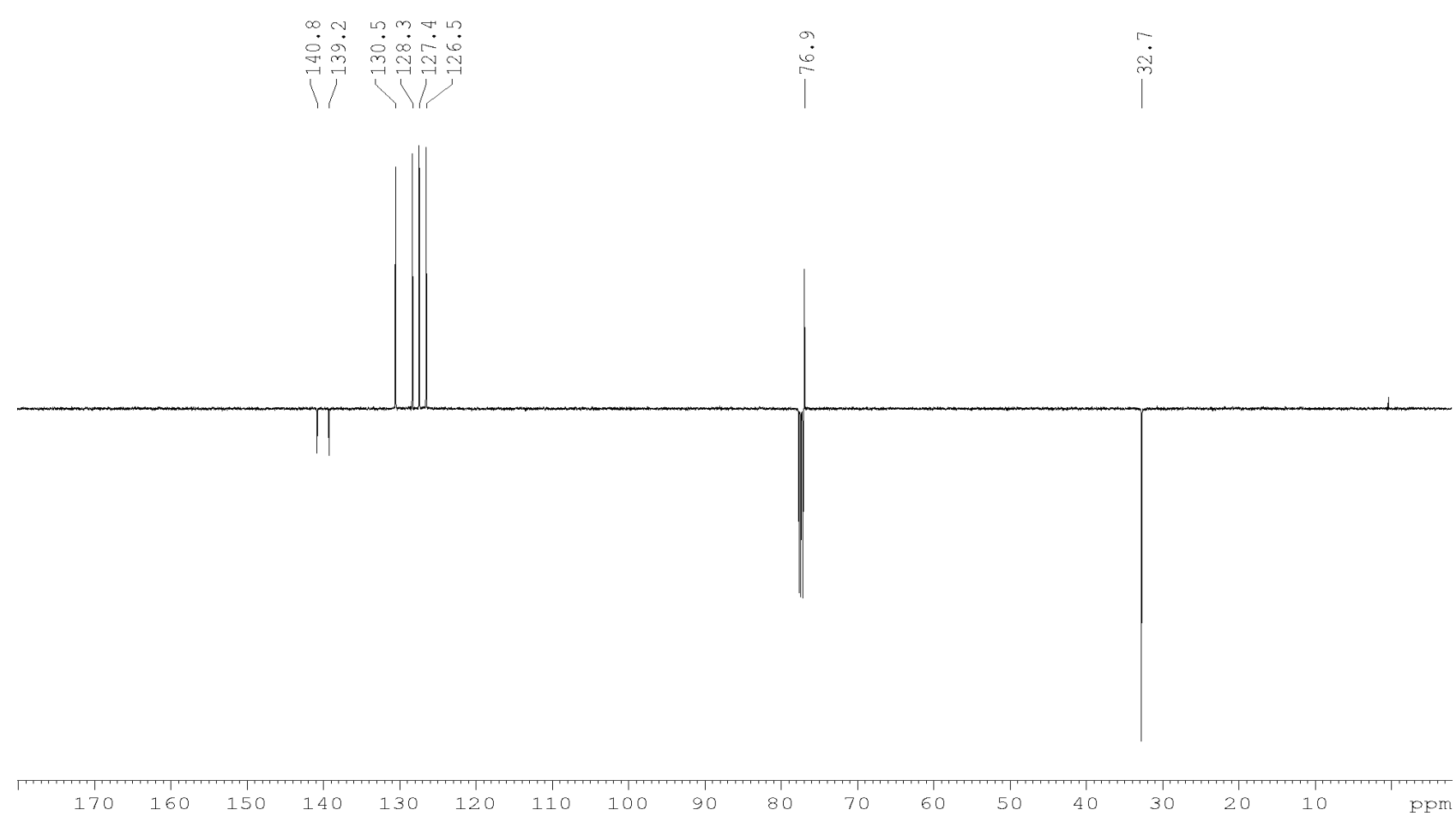
${ }^{1} \mathrm{H}$ NMR $\left(500 \mathrm{MHz}, \mathrm{CDCl}_{3}, 298 \mathrm{~K}\right)$ of 2,3-dihydro- $1 H$-inden-1-ol, $\mathbf{5 m}$

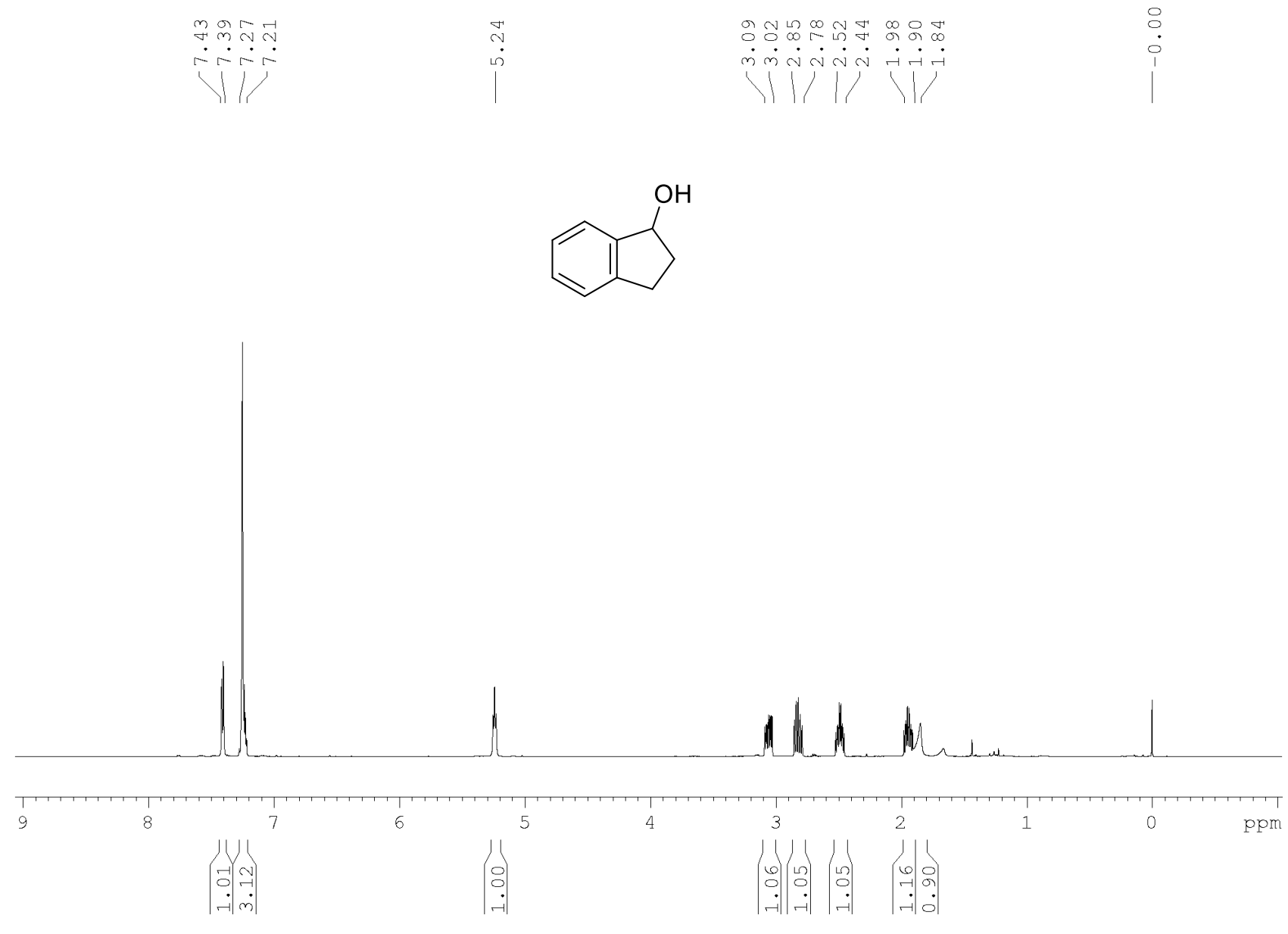

${ }^{13} \mathrm{C}-\left\{{ }^{1} \mathrm{H}\right\}$ NMR (126 MHz, $\left.\mathrm{CDCl}_{3}, 298 \mathrm{~K}\right)$ of 2,3-dihydro- $1 H$-inden-1-ol, $\mathbf{5 m}$

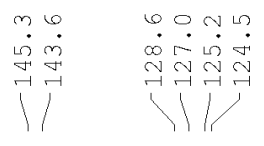

$\stackrel{\infty}{\dot{0}} \quad \dot{m}_{i}^{\infty}$

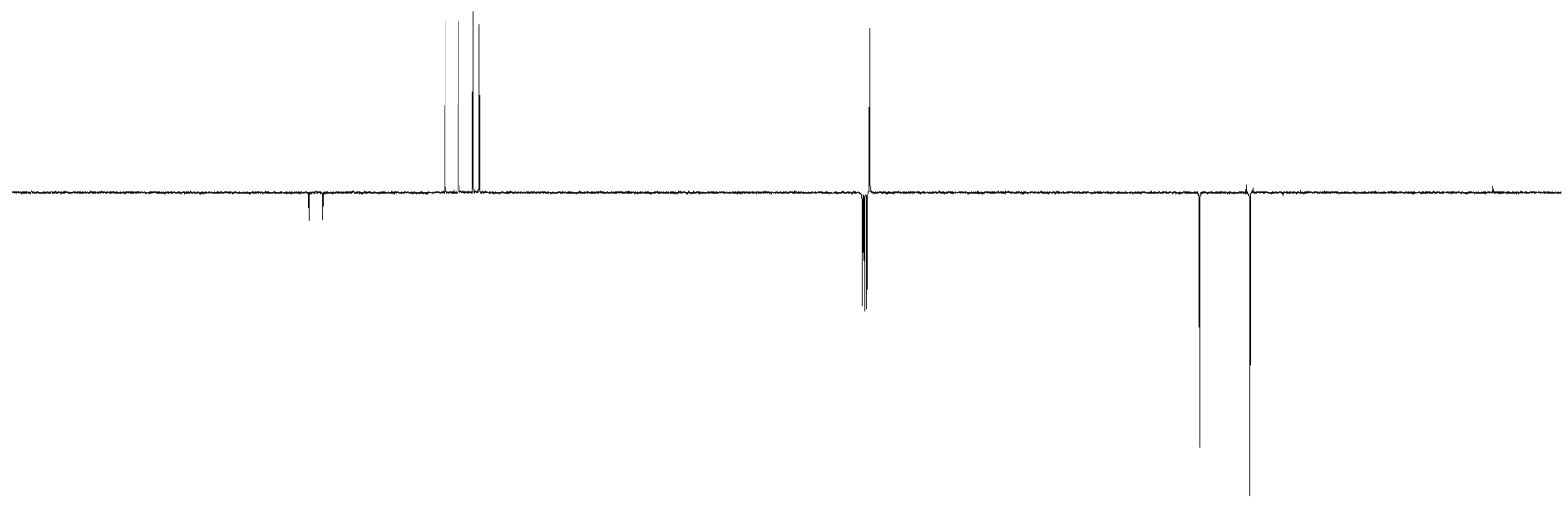

$\begin{array}{lllllllllllllllll}170 & 160 & 150 & 140 & 130 & 120 & 110 & 100 & 90 & 80 & 70 & 60 & 50 & 40 & 30 & 20 & 10\end{array}$ 
${ }^{1} \mathrm{H}$ NMR $\left(500 \mathrm{MHz}, \mathrm{CDCl}_{3}, 298 \mathrm{~K}\right)$ of 1-(pyridin-2-yl)ethan-1-ol, 5n

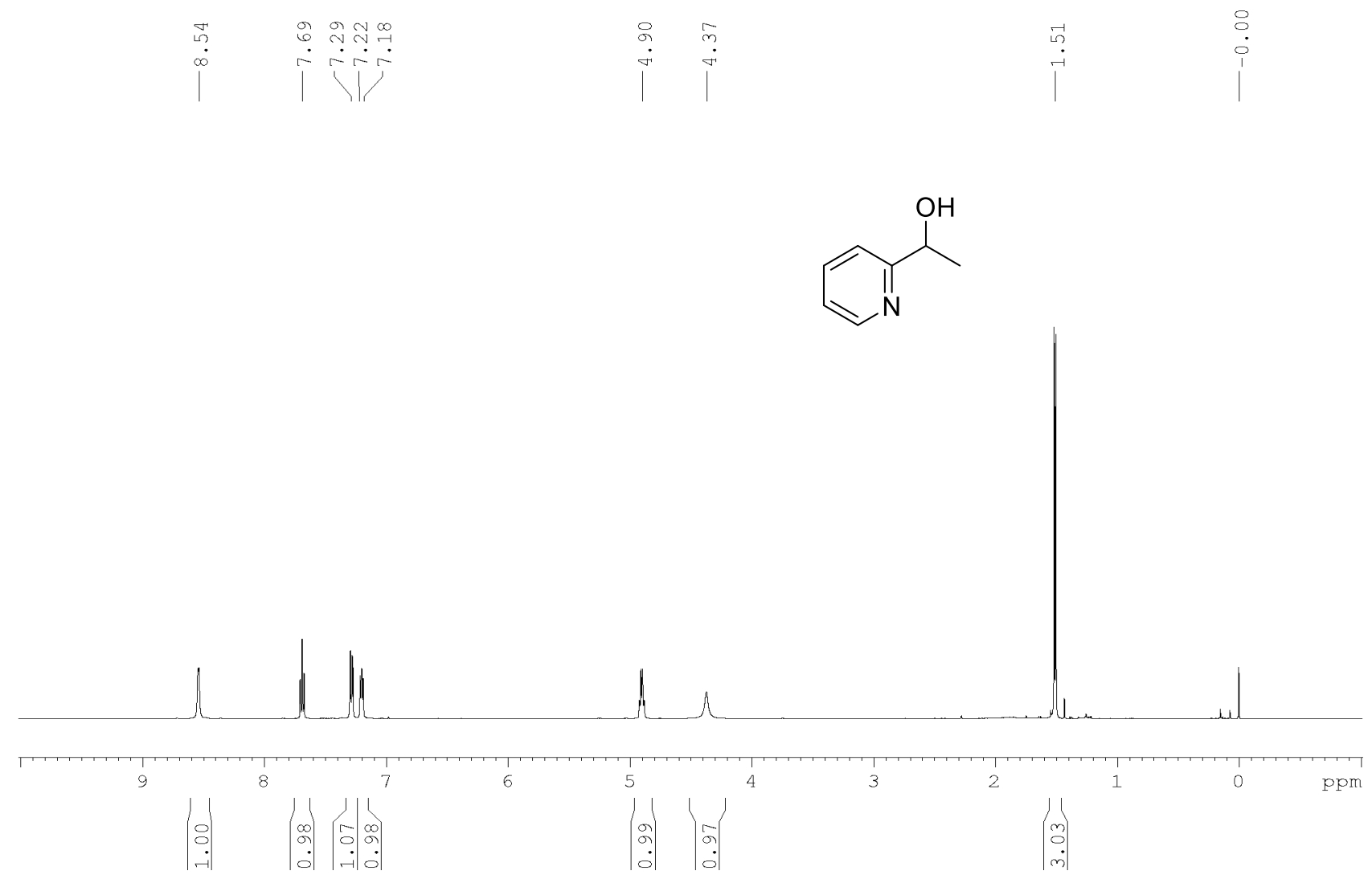

${ }^{13} \mathrm{C}-\left\{{ }^{1} \mathrm{H}\right\}$ NMR $\left(126 \mathrm{MHz}, \mathrm{CDCl}_{3}, 298 \mathrm{~K}\right)$ of 1-(pyridin-2-yl)ethan-1-ol, $\mathbf{5 n}$

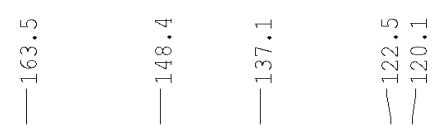
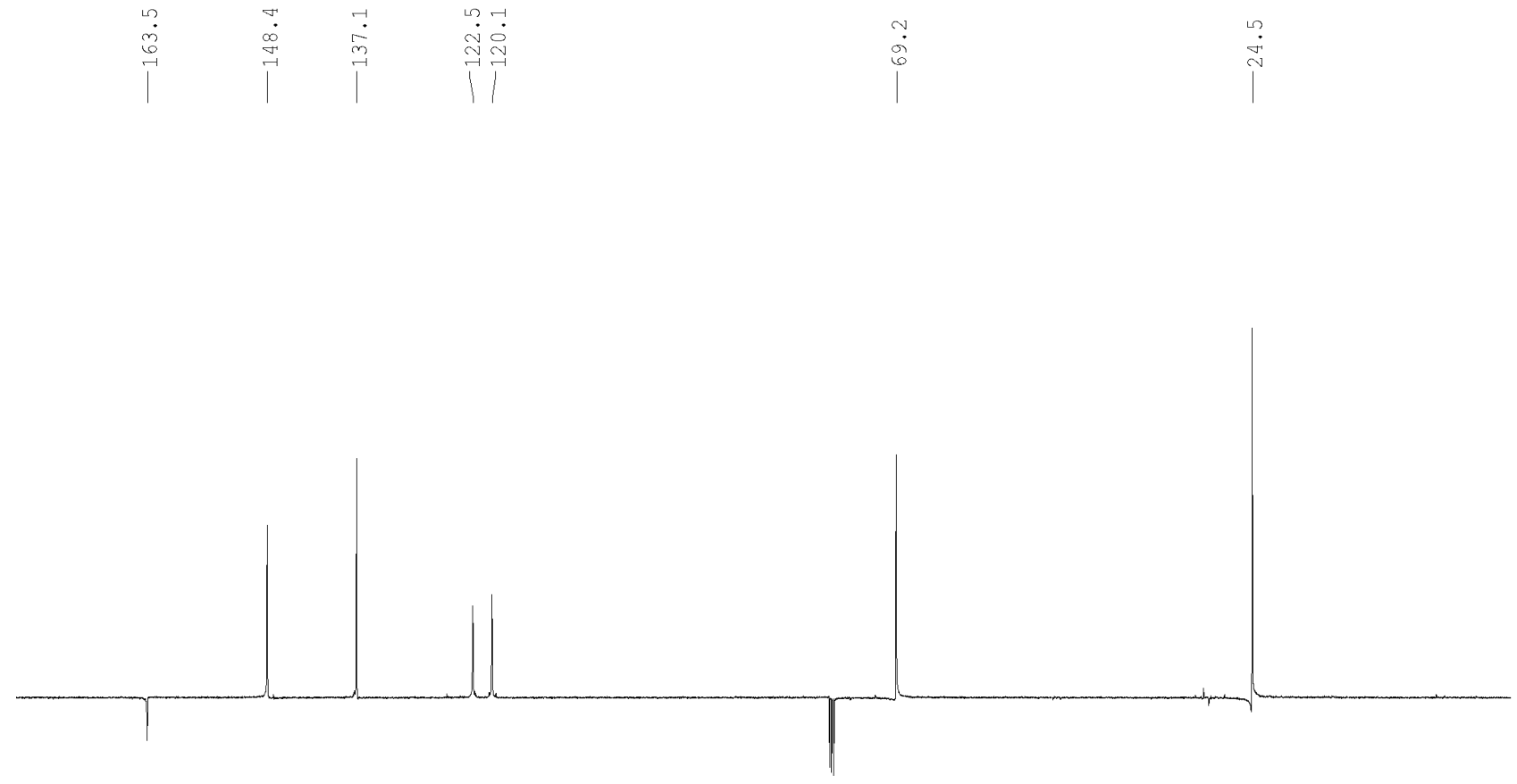

$\begin{array}{lllllllllllllllllllllllll}170 & 160 & 150 & 140 & 130 & 120 & 110 & 100 & 90 & 80 & 70 & 60 & 50 & 40 & 30 & 20 & 10 & 0 & 0\end{array}$ 
${ }^{1} \mathrm{H}$ NMR $\left(500 \mathrm{MHz}, \mathrm{CDCl}_{3}, 298 \mathrm{~K}\right)$ of nonan-5-ol, $\mathbf{5 0}$

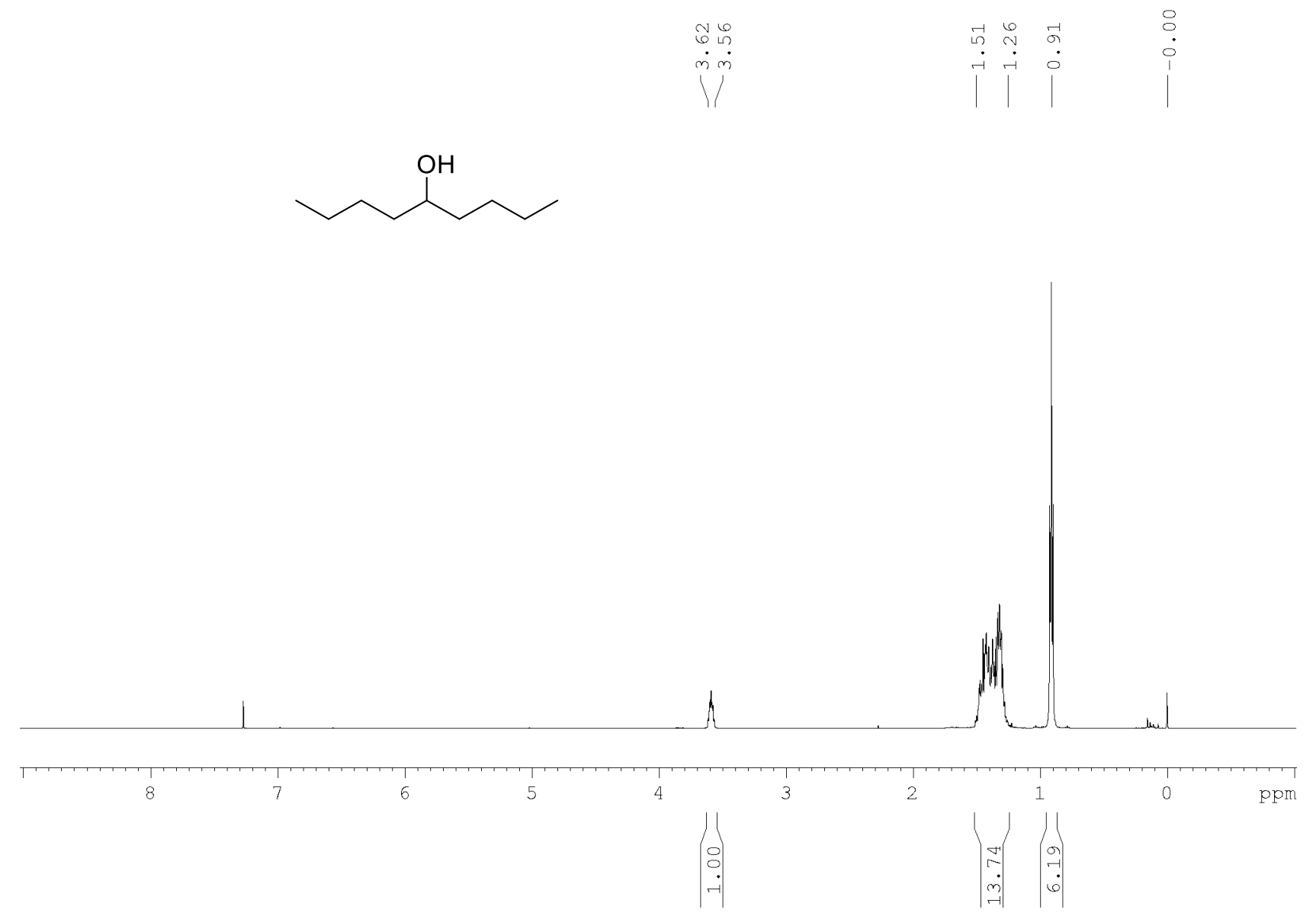

${ }^{13} \mathrm{C}-\left\{{ }^{1} \mathrm{H}\right\}$ NMR $\left(126 \mathrm{MHz}, \mathrm{CDCl}_{3}, 298 \mathrm{~K}\right)$ of nonan-5-ol, $\mathbf{5 0}$
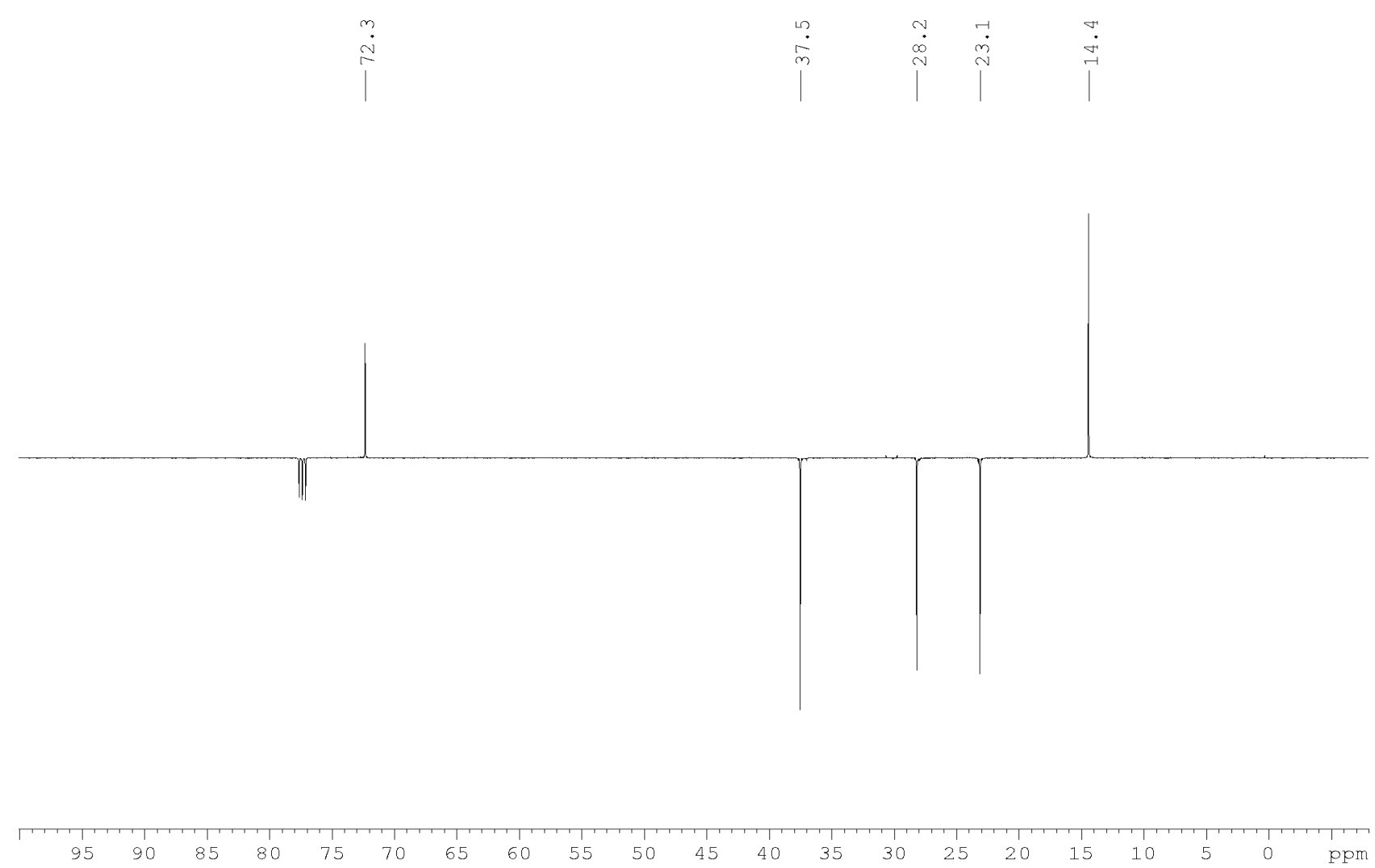

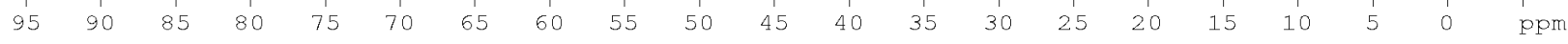


${ }^{1} \mathrm{H}$ NMR $\left(500 \mathrm{MHz}, \mathrm{CDCl}_{3}, 298 \mathrm{~K}\right)$ of 4-phenylbutan-2-ol, 5p

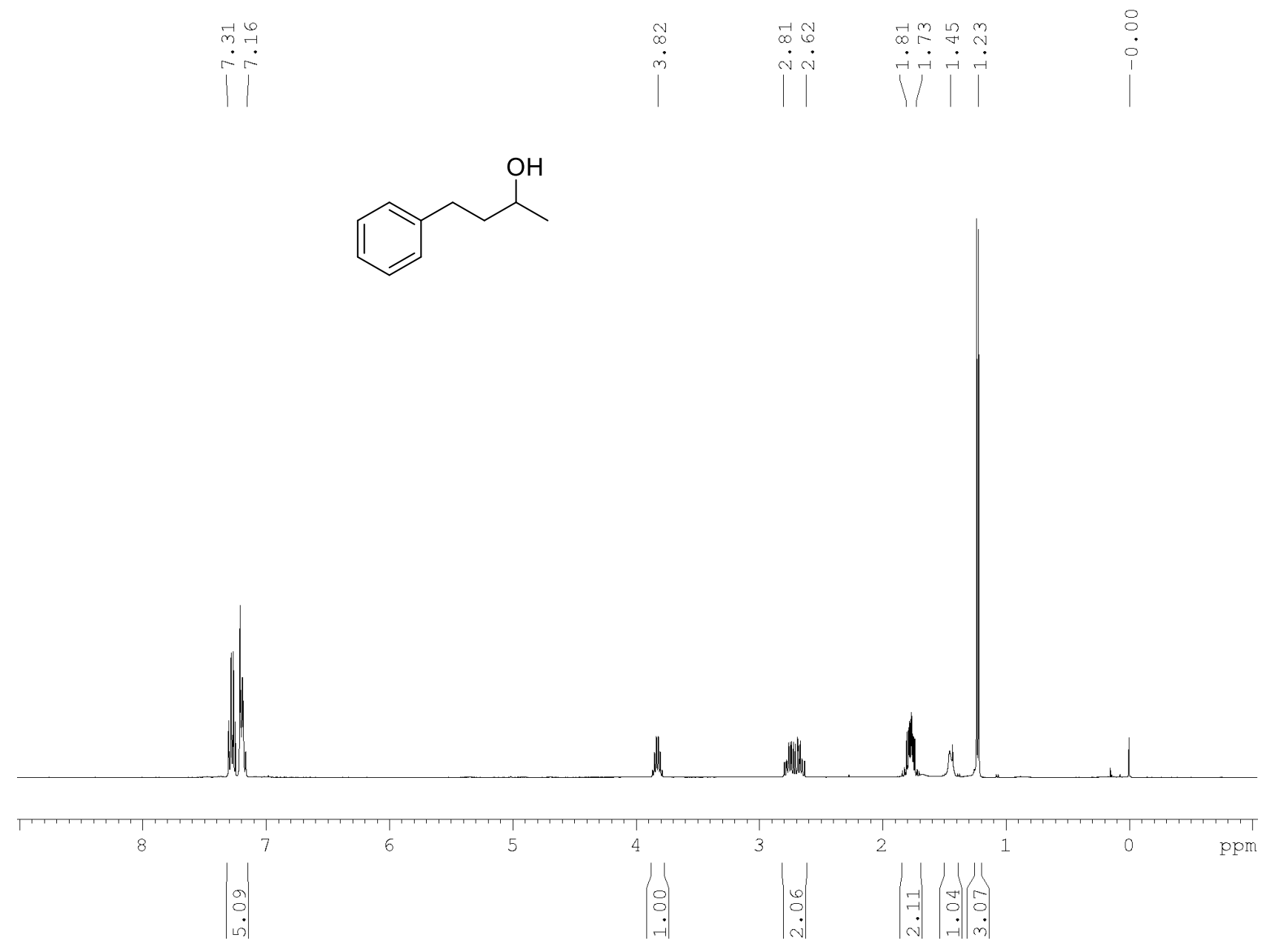

${ }^{13} \mathrm{C}-\left\{{ }^{1} \mathrm{H}\right\}$ NMR (126 MHz, $\left.\mathrm{CDCl}_{3}, 298 \mathrm{~K}\right)$ of 4-phenylbutan-2-ol, 5p

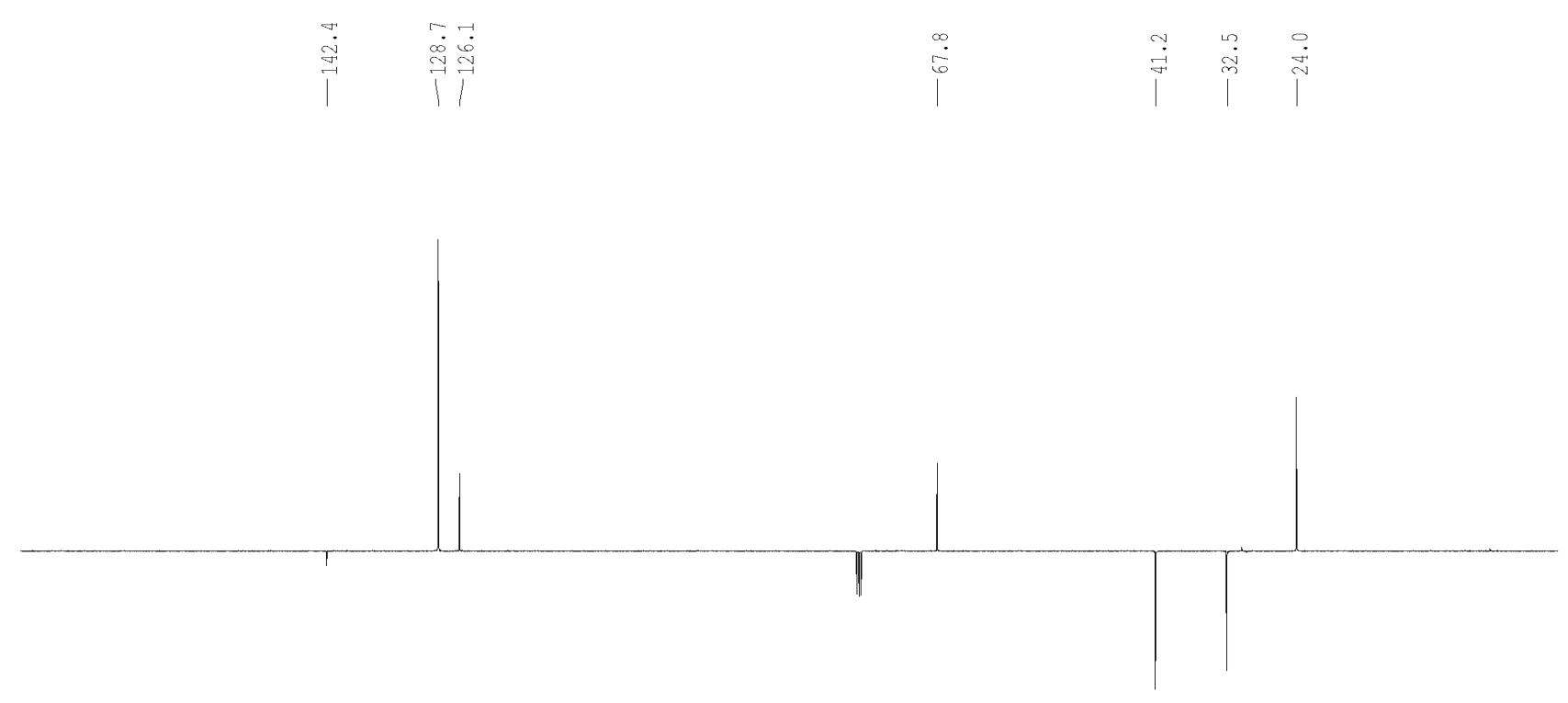

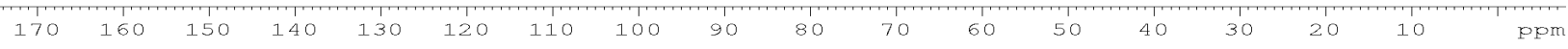


${ }^{1} \mathrm{H}$ NMR (500 MHz, $\left.\mathrm{CDCl}_{3}, 298 \mathrm{~K}\right)$ of 1-(adamantan-2-yl)ethan-1-ol, 5q<smiles>CC(O)C1C2CC3CC(C2)CC1C3</smiles>

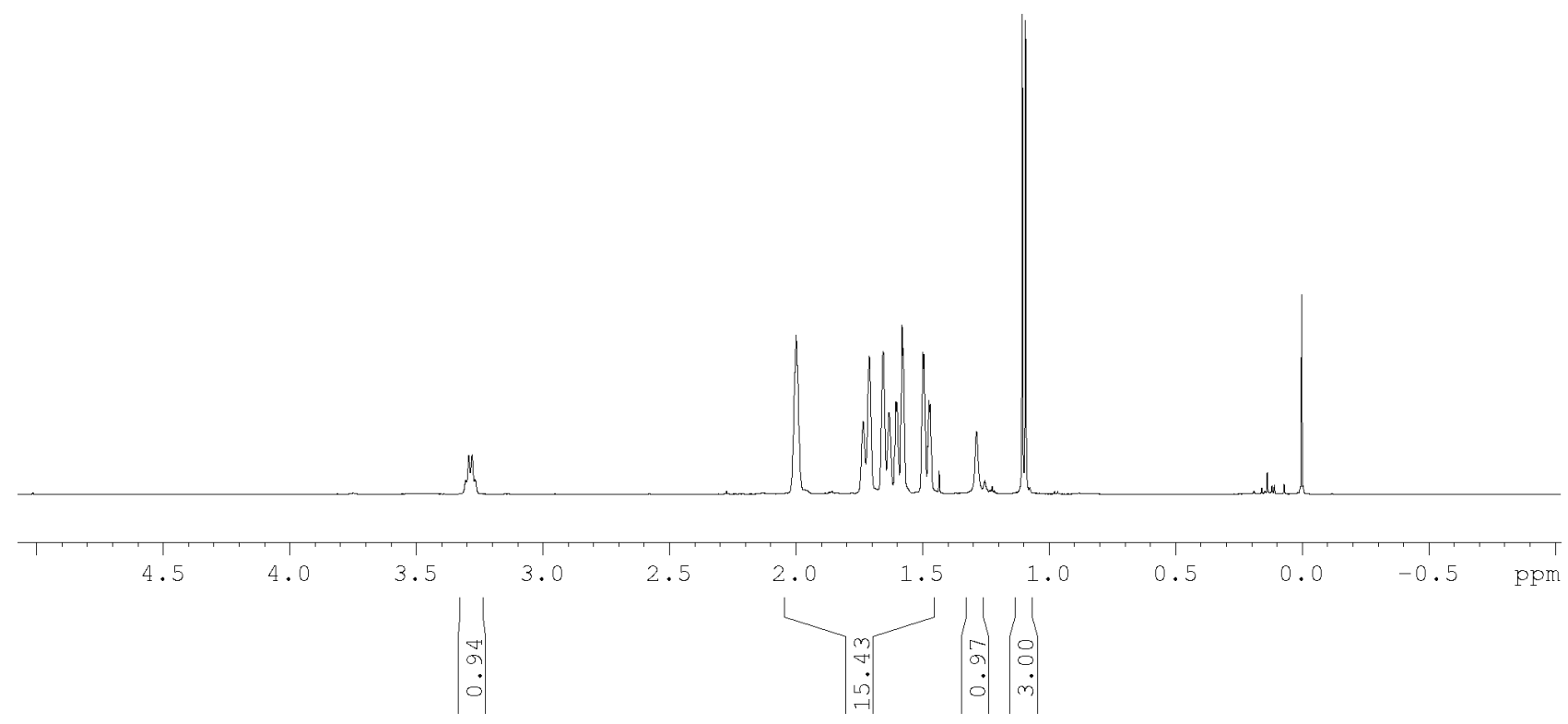

${ }^{13} \mathrm{C}-\left\{{ }^{1} \mathrm{H}\right\}$ NMR (126 MHz, $\left.\mathrm{CDCl}_{3}, 298 \mathrm{~K}\right)$ of 1-(adamantan-2-yl)ethan-1-ol, 5q
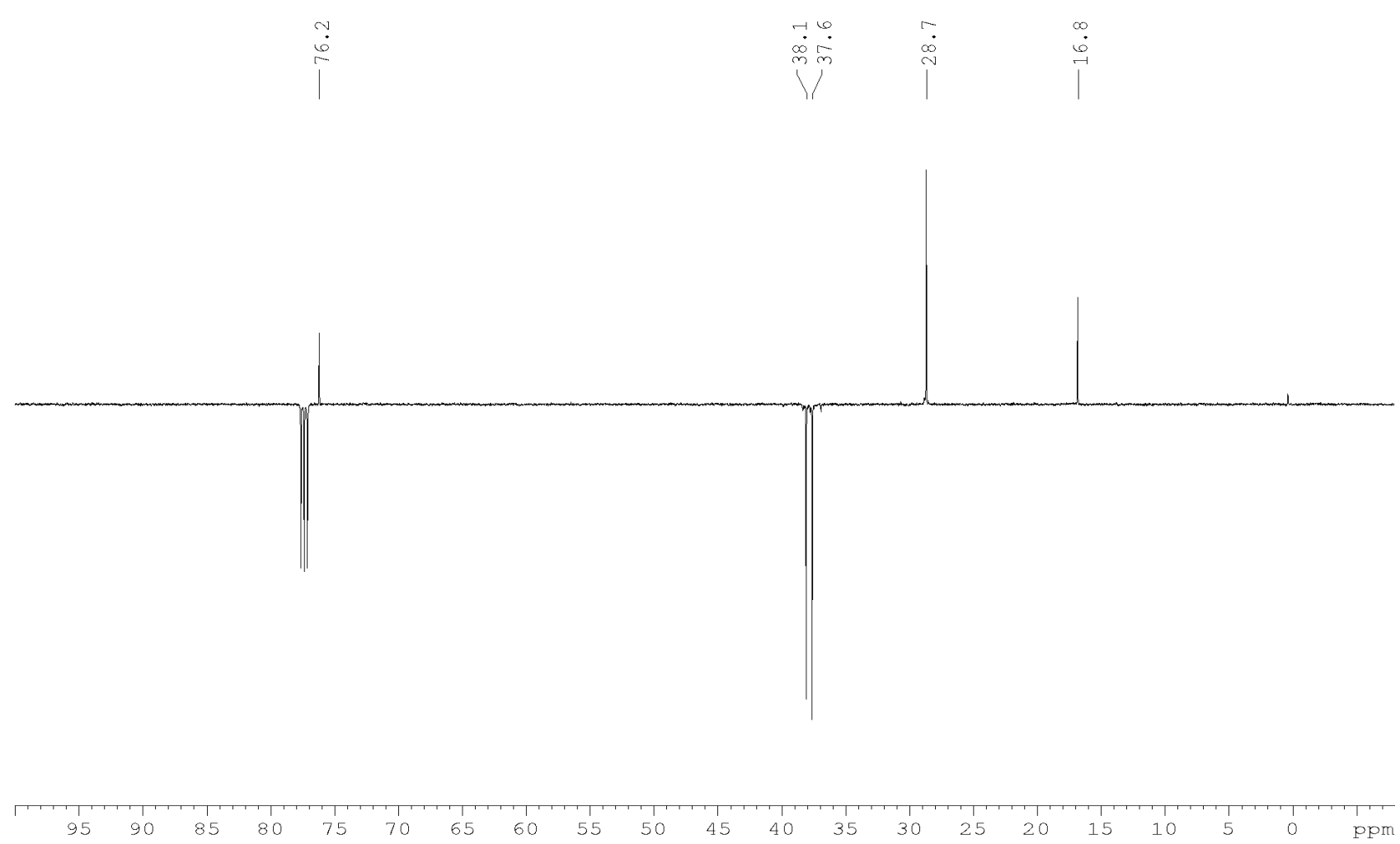
${ }^{1} \mathrm{H}$ NMR $\left(500 \mathrm{MHz}, \mathrm{CDCl}_{3}, 298 \mathrm{~K}\right)$ of adamantan-2-ol, $\mathbf{5 r}$

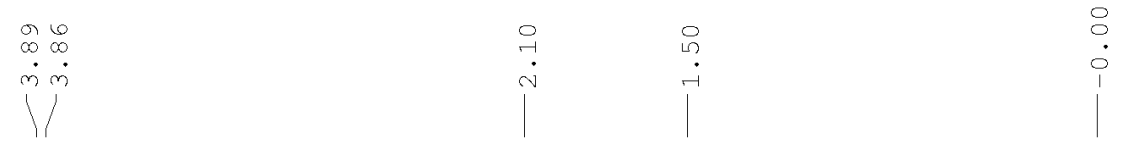

$N^{O H}$

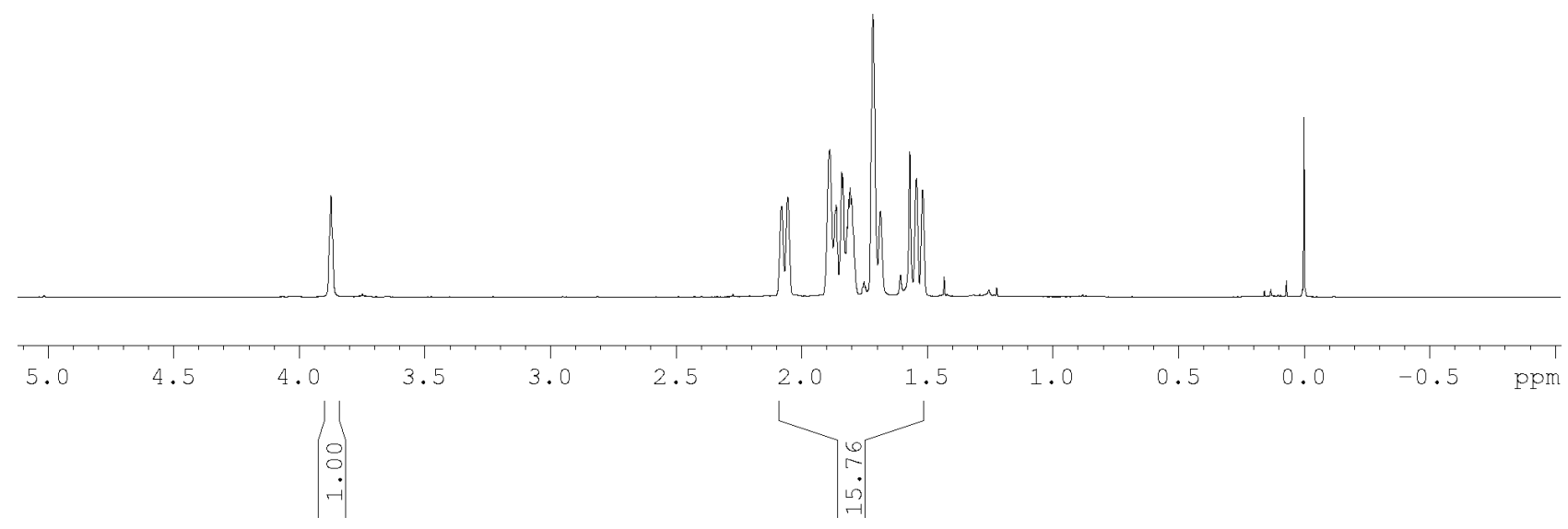

${ }^{13} \mathrm{C}-\left\{{ }^{1} \mathrm{H}\right\}$ NMR (126 MHz, $\left.\mathrm{CDCl}_{3}, 298 \mathrm{~K}\right)$ of adamantan-2-ol, $\mathbf{5 r}$
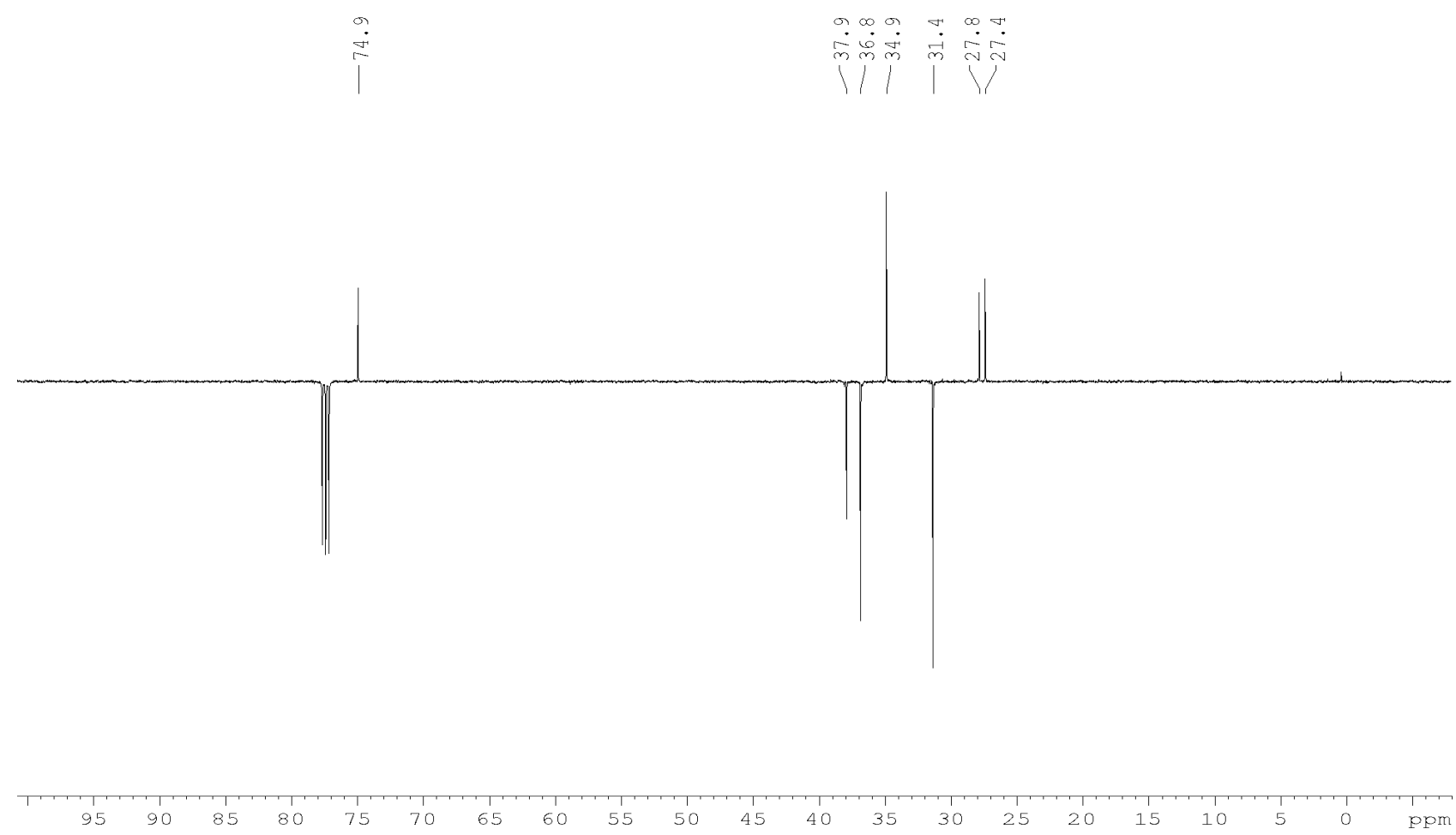


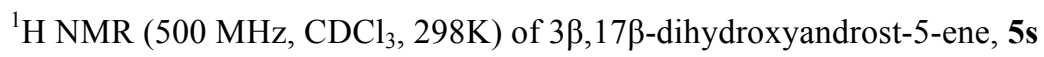

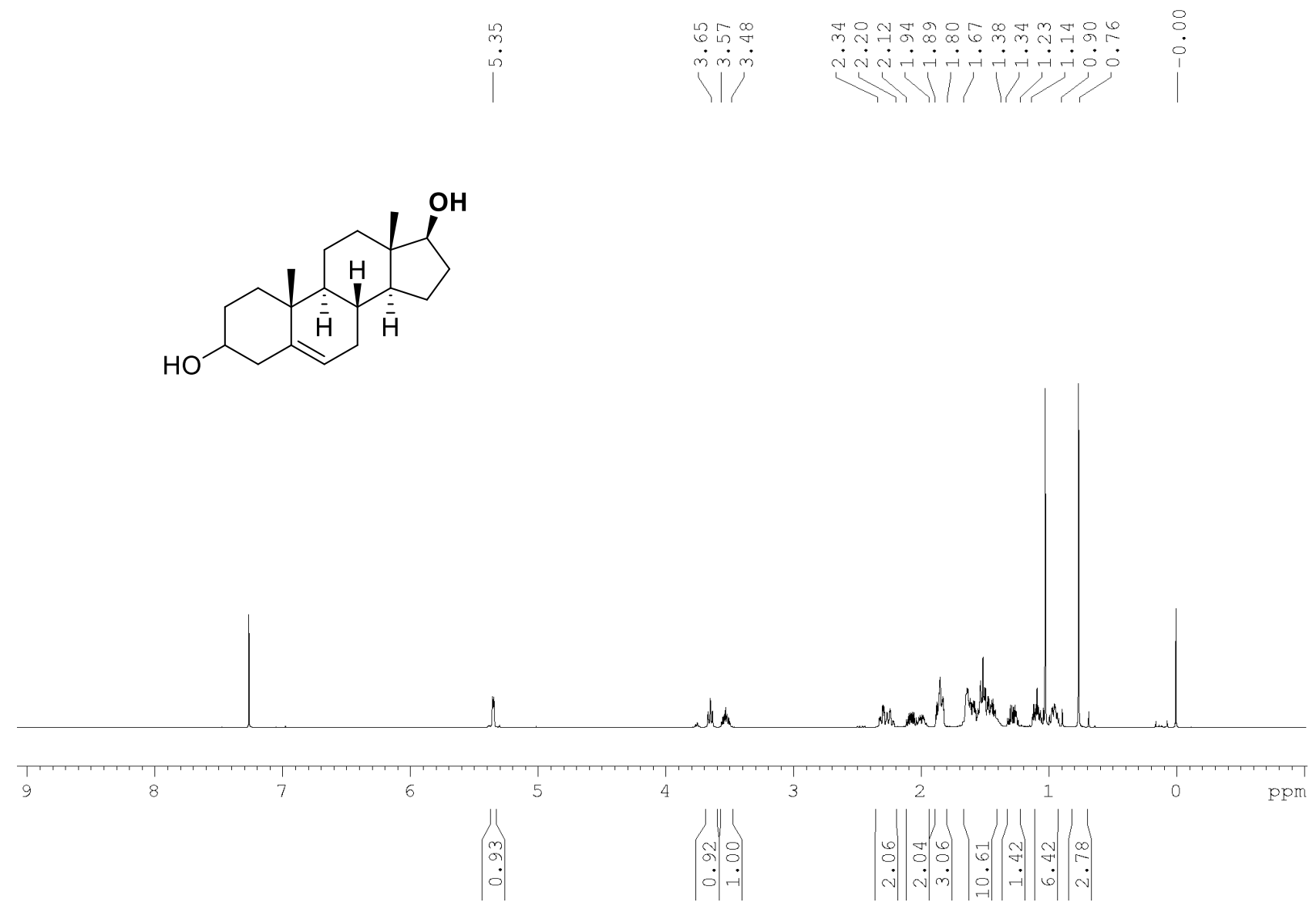

${ }^{13} \mathrm{C}-\left\{{ }^{1} \mathrm{H}\right\}$ NMR $\left(126 \mathrm{MHz}, \mathrm{CDCl}_{3}, 298 \mathrm{~K}\right)$ of $3 \beta, 17 \beta$-dihydroxyandrost-5-ene, $\mathbf{5 s}$
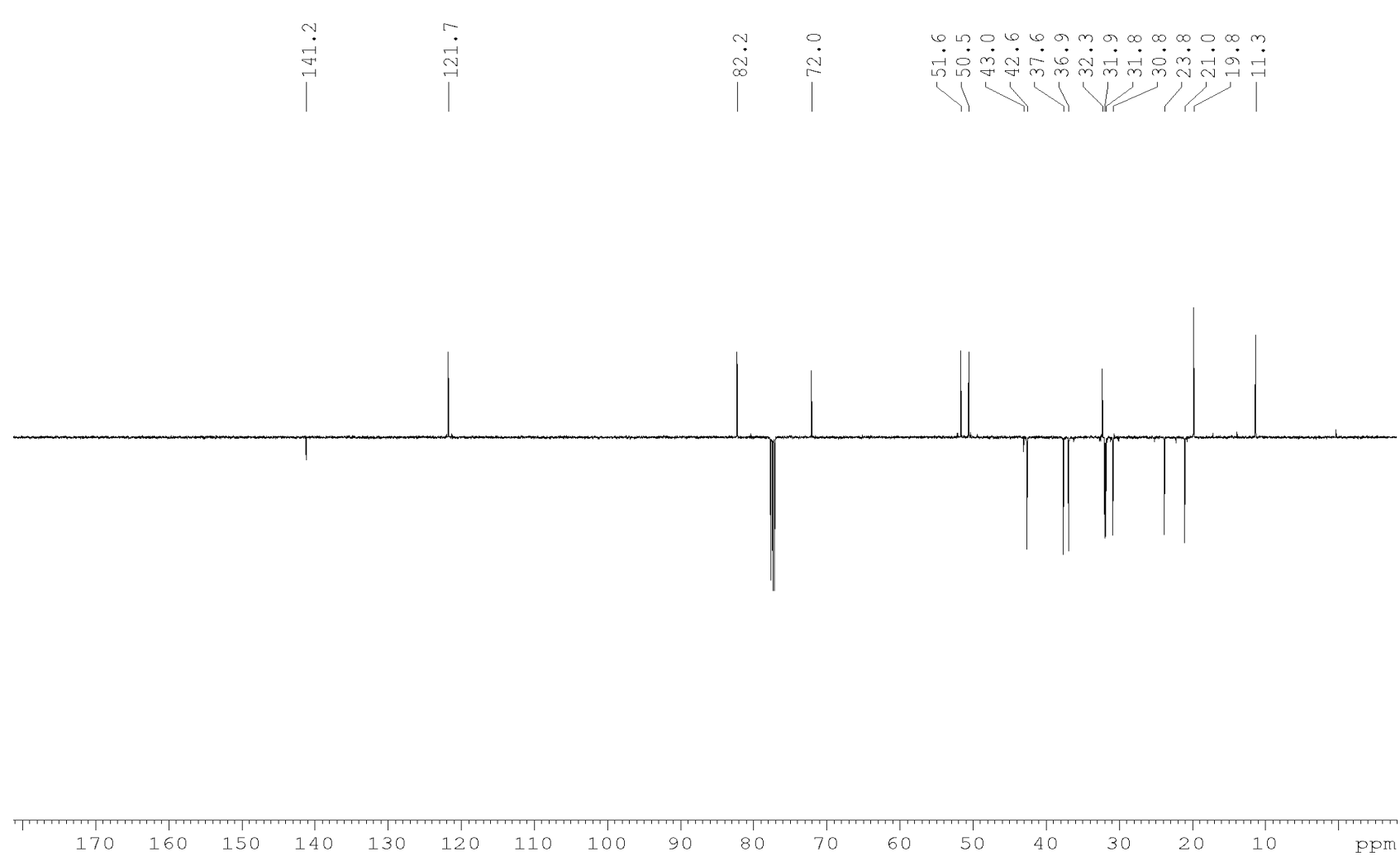


\section{References}

[1] F. S. Wekesa, R. Arias-Ugarte, L. Kong, Z. Sumner, G. P. McGovern, M. Findlater, Organometallics, 2015, 34, 5051-5056.

[2] B. Martín-Matute, M. Edin, K. Bogár, F. B. Kaynak, Jan-E. Bäckvall, J. Am. Chem. Soc. 2005, 127, 8817-8825.

[3] J. L. N. Fernandes, M. C. de Souza, E. C. S. Brenelli, J. A. Brenelli, Synthesis, 2009, 23, 4058-4062.

[4] L. C. M. Castro, D. Bézier, J.-B. Sortais, C. Darcela, Adv. Synth. Catal. 2011, 353, 1279-1284.

[5] S. Koul, J. L. Koul, B. Singh, M. Kapoor, R. Parshad, K. S. Manhas, S. C. Taneja, G. N. Qazi, Tetrahedron: Asymmetry, 2005, 16, 2575-2591.

[6] M. Kuriyama, R. Shimazawa, R. Shirai, J. Org. Chem. 2008, 73, 1597-1600.

[7] S. Raha Roy, S. Chandra Sau, S. K. Mandal, J. Org. Chem. 2014, 79, 9150-9160.

[8] B. Denegri, O. Kronja, J. Org. Chem. 2007, 72, 8427-8433.

[9] W. Weissensteiner, O. Hofer, U. G. Wagner, J. Org. Chem. 1988, 53, 3988-3996.

[10] T. Iwasaki, K. Agura, Y. Maegawa, Y. Hayashi, T. Ohshima, K. Mashima, Chem. Eur. J. 2010, 16, $11567-11571$.

[11] P. N. Liu, K. D. Ju, C. P. Lau, Adv. Synth. Catal. 2011, 353, 275-280.

[12] I. P. Query, P. A. Squier, E. M. Larson, N. A. Isley, T. B. Clark, J. Org. Chem. 2011, 76, 6452-6456.

[13] K. Kamata, K. Yonehara, Y. Nakagawa, K. Uehara, N. Mizuno, Nat. Chem. 2010, 2, 478-483.

[14] a) M. I. Choudhary, S. A. A. Shah, S. G. Musharraf, F. Shaheen, A.-U.-Rahman, Nat. Prod. Res. 2003, 17, $215-$ 220; b) H. -P. Li, P. Yu, H. -J. Zhang, H.-M. Liu, Chin. J. Chem. 2008, 26, 1666-1668. 